University of Louisville ThinkIR: The University of Louisville's Institutional Repository

Electronic Theses and Dissertations

$12-2015$

\title{
Mechanisms and intervention strategies for alcohol and HIV-antiretroviral therapy-induced liver injury.
}

Hridgandh Donde

University of Louisville

Follow this and additional works at: https://ir.library.louisville.edu/etd

Part of the Pharmacology Commons, Toxicology Commons, and the Translational Medical $\underline{\text { Research Commons }}$

\section{Recommended Citation}

Donde, Hridgandh, "Mechanisms and intervention strategies for alcohol and HIV-antiretroviral therapy-induced liver injury." (2015). Electronic Theses and Dissertations. Paper 2317.

https://doi.org/10.18297/etd/2317

This Doctoral Dissertation is brought to you for free and open access by ThinkIR: The University of Louisville's Institutional Repository. It has been accepted for inclusion in Electronic Theses and Dissertations by an authorized administrator of ThinkIR: The University of Louisville's Institutional Repository. This title appears here courtesy of the author, who has retained all other copyrights. For more information, please contact thinkir@louisville.edu. 


\title{
MECHANISMS AND INTERVENTION STRATEGIES FOR ALCOHOL AND
} HIV-ANTIRETROVIRAL THERAPY-INDUCED LIVER INJURY

\section{By}

\author{
Hridgandh Donde \\ M.Sc., University of Mumbai, India 2010 \\ M.S., University of Louisville, Kentucky 2013
}

A Dissertation Submitted to the Faculty of the School of Medicine of the University of Louisville in Partial fulfillment of the Requirements

For the Degree of

\author{
Doctor of Philosophy \\ In Pharmacology and Toxicology
}

Department of Pharmacology and Toxicology

University of Louisville

Louisville, KY

December 2015 
Copyright 2015 by Hridgandh Donde

CAIl rights reserved 

MECHANISMS AND INTERVENTION STRATEGIES FOR ALCOHOL AND HIVANTIRETROVIRAL THERAPY-INDUCED LIVER INJURY

By

Hridgandh Donde

Dissertation Approved on

November $9^{\text {th }} 2015$

By the following Dissertation Committee:

Shirish Barve, Ph.D.

Craig McClain, M.D.

Swati Joshi-Barve, Ph.D.

Matthew C. Cave, M.D.

LaCreis Kidd, Ph.D 


\section{ACKNOWLEDGEMENTS}

First and foremost, I would like to appreciate and thank Dr. Shirish Barve, my mentor for the continuous support in my Ph.D. study and related research, for his patience and motivation. Your advice on both research as well as on my career have been invaluable. I appreciate all your efforts, feedbacks and contributions of time, ideas and funding to make my Ph.D. experience productive and stimulating. It has been an absolute pleasure working with you and I am thankful for the excellent example you have provided as an independent investigator and a critical thinker.

I would like to thank my dissertation committee, Dr. Craig McClain, Dr. SwatiJoshi Barve, Dr. Matthew Cave and Dr. LaCreis Kidd for all your valuable feedbacks, suggestions and comments on my dissertation work. The annual committee meetings were extremely productive with new ideas, suggestions and different perspectives. Dr. McClain- I would like to thank you for all the funding and grants support you provided. This has immensely helped me in carrying out my research work more efficiently for all 5 years.

I am thankful to all the members of the Barve lab - Dr. Swati Barve, thank you all your suggestions and inputs you provided. Thank you for teaching me all the nitty gritty and the basics of the research work I leant in the lab. Special thanks Dr. Smita Ghare for teaching me lab techniques especially for my in-vitro studies. 
Also, I would like to thank you for all your suggestions during thesis writing. Thank you Jing Zhang for the invaluable help in the animal studies. A big thank you to Dr. Barker, Dr. Gobejishvili and Dr. Kirpich for sharing all your expertise, suggestions and comments that truly contributed in completing my dissertation work. I would also like to thank all the past and present fellow labmates of the Barve lab. It was always fun and enjoyable working with you guys.

I would like to thank our department chair Dr. Hein and all the members of the department of Pharmacology and Toxicology. It has been a pleasure working with you all.

Lastly, I would like to thank my family and friends for their love, encouragement and support through all my ups and downs during these 5 years. I have still not found words to express my gratitude towards my parents for their love, patience and support. I would not have had the courage to embark on this journey without their support.

Hridgandh 


\section{ABSTRACT \\ MECHANISMS AND INTERVENTION STRATEGIES FOR ALCOHOL AND HIV-ANTIRETROVIRAL THERAPY-INDUCED LIVER INJURY \\ Hridgandh Donde \\ November $9^{\text {th }} 2015$}

Section I: Chronic alcohol consumption is a leading cause of liver disease and liver-related death worldwide. Alcoholic liver disease includes, hepatic steatosis, steatohepatitis and ultimately fibrosis and cirrhosis. Emerging evidence has established the important role of the "gut-liver" axis in the development of alcoholic liver disease (ALD). Our recent work indicated that chronic alcohol induced perturbations in the gut microbiome and consequent changes in fatty acids have a major impact on the development of intestinal barrier dysfunction and ALD. The aim of this study was to investigate whether treatment with tributyrin - a butyrate prodrug results in protection against ALD in terms of hepatic steatosis, inflammation and injury. Tributyrin is a triglyceride that is rapidly absorbed and metabolized to butyrate. Moreover, it has more favorable pharmacokinetics compared with butyrate with low toxicity.

In the present study, we have used a mouse model of ALD to examine the effects of Tributyrin oral administration on ethanol-induced changes in intestinal permeability and hepatic steatosis, inflammation and injury. 8-10-week old C57BL/6 male mice were chronically pair-fed the Lieber-DeCarli liquid diet 
containing alcohol or isocaloric maltose dextrin. Tributyrin was administered to a sub-group of alcohol-fed animals by oral gavage $(2 \mathrm{~g} / \mathrm{kg})$ for 5 days/week to assess its effects.

Tributyrin attenuated the ethanol-induced gut barrier dysfunction, as shown by the significant reduction in endotoxemia. Mechanisms of chronic alcohol-induced gut-barrier dysfunction were examined by immunohistochemical analyses of the ileum section of mice chronically fed alcohol. Specifically, alcohol induced a robust down-regulation of intestinal tight junction proteins $\mathrm{ZO}-1$ and occludin which was markedly attenuated by tributyrin administration. Liver histological analysis showed a significant decrease in ethanol-induced hepatic steatosis. Tributyrin also prevented the ethanol-induced down-regulation of hepatic CPT-1, a key enzyme in free fatty acid $\beta$-oxidation, with a resultant decrease in hepatic triglycerides and free fatty acids. To further elucidate the mechanism by which tributyrin prevents hepatic steatosis, we performed experiments using primary hepatocytes. Hepatocytes were treated with alcohol and with/without pre-treatment with butyrate. Alcohol-induced downregulation of CPT-1 gene expression was significantly prevented by butyrate. Butyrate is a known HDAC inhibitor. Hence promoter histone acetylation state was investigated. In this regard, acetylation of histone 3 lysine 9 (H3K9), a critical feature of the active promoter state was significantly increased by butyrate and was essentially mediated by the p300-histone acetyltransferase. Increased acetylation leads to an open chromatin configuration causing increased recruitment of co-activators and TF such as PGC-1 and Sp-1 at the CPT-1 
promoter. Additionally RNA-Pol II binding was also increased by butyrate pretreatment in alcohol treated hepatocytes. These data suggest that butyrate, by acting as an HDAC inhibitor, induces promoter histone acetylation leading to the transcriptional activation of CPT-1 under pathologic conditions of alcohol exposure.

Butyrate has been shown to have beneficial effects by decreasing obesityassociated inflammation, and insulin resistance. Hence, its effects on alcohol induced hepatic inflammation were also investigated. F4/80 staining of liver sections revealed increased macrophage infiltration in 2 weeks of alcohol feeding whereas tributryrin administration markedly blocked macrophage infiltration. Tributyrin administration attenuated the ethanol mediated pro-inflammatory cytokine TNF- $\alpha$ mRNA expression. Alcohol-induced neutrophil infiltration was also significantly suppressed by tributyrin administration. Neutrophil infiltration was further confirmed by MPO activity. Tributyrin administered mice had a significant drop in MPO activity as compared to alcohol-fed mice at 7 weeks but not in 2 weeks. Inflammatory chemokine and cytokine such as CXCL-2 (MCP-1) and CCL-2 (MIP-2) mRNA were significantly upregulated in alcohol-fed mice and tributryrin administration significantly attenuated alcohol-induced induction of these pro-inflammatory chemokines. Notably, tributryrin also significantly attenuated liver injury as seen by a decrease in serum AST \& ALT levels. Hence, the present work demonstrates that tributyrin may be useful in preventing the ethanol-induced pathogenic changes in the "gut-liver" axis and may prove to be a useful therapy for the prevention/treatment of ALD. 
Section II: HIV protease inhibitors (HIV-PIs) are the major components of the highly active anti-retroviral therapy (HAART) and have been successfully used in the treatment of HIV-1 infection in the past two decades. However prolonged use of HAART is known to cause hepatotoxicity. Hence it is highly relevant to study the underlying mechanisms in HAART induced hepatotoxicity that can negatively impact treatment outcomes. One of the known mechanisms of hepatotoxicity caused due to HIV-PIs drugs is endoplasmic reticulum (ER) stress and subsequent activation of unfolded protein response (UPR) leading to dysregulation of hepatic lipid metabolism. Our recent work shows that PDE4/CAMP metabolism plays a significant role in alcohol-induced hepatic steatosis and injury. Hence in the present study, we examined the potential mechanisms underlying HIV-PI induced hepatic ER stress and toxicity with a particular emphasis on the pathogenic role of PDE4 family of enzymes.

The effects of clinically used HIV-PIs [Ritonavir and Lopinavir] were examined both in a rat hepatoma cell line (H4IIE). The data obtained from these studies demonstrated that Ritonavir and Lopinavir in combination led to a significant loss of hepatocyte survival. Notably, inhibition of PDE4 by a specific PDE4-inhibitor, rolipram, markedly attenuated hepatotoxicity induced by PI treatments. Examination of the mechanistic role of PDE4 showed that PDE4 inhibition significantly decreases the PIs-induced expression of the ER stress related proteins $\mathrm{CHOP}, \mathrm{ATF}-4$ and -3 . Furthermore, examination of the PDE4/cAMP-regulated downstream signaling demonstrated the involvement of cAMP PKA (protein kinase A). Hence, effect of $\mathrm{H}-89$ (PKA inhibitor) was 
examined in hepatocytes treated with HIV-PI and rolipram. PKA inhibition significantly reversed the rolipram protective effect leading to re-expression of ER-stress related genes.

Fas/FasL signaling plays a critical role in drug-induced hepatotoxicity and cell death Hence activation of hepatotoxic Fas/FasL signaling in HIV-PI induced hepatotoxicity was investigated. Combination of Rit+Lop significantly upregulated FasL and Fas expression in H4IIE rat hepatoma cell line.

Commensurate with the increase in FasL and Fas, PDE4 expression was also increased with Rit+Lop treatment. Notably PDE4 inhibition down-regulated Fas and FasL mRNA and protein expression induced by HIV PIs whereas PKA inhibitor H-89 significantly reversed the rolipram effect on Fas and FasL expression. Taken together, these data strongly support and identify a pathogenic/mechanistic role for PDE4 regulated cAMP-PKA in the development of HIV-PI induced ER-stress and FasL mediated cell death leading to hepatic steatosis and injury. 


\section{TABLE OF CONTENTS}

\section{PAGE}

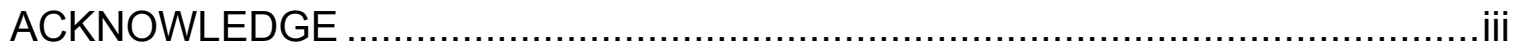

ABSTRACT …

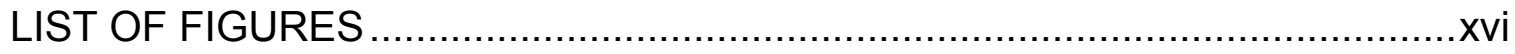

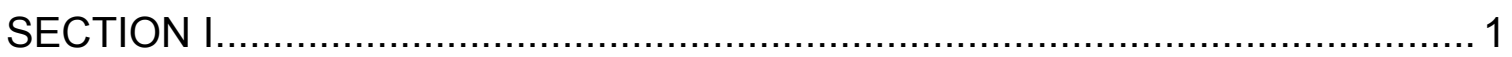

INTRODUCTION

Alcoholic Liver Disease - General Overview ............................................... 2

Alcohol-induced Hepatic Steatosis (fatty liver) and its Mechanisms ................... 3

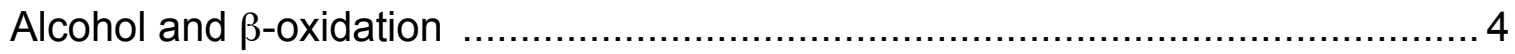

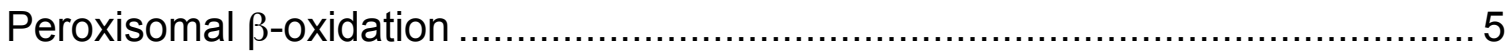

Regulation of CPT-1 gene expression and function: Relevance to alcohol-

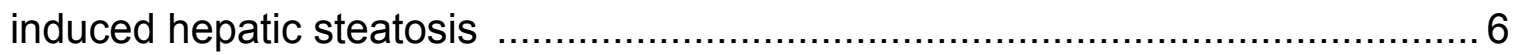

Role of Peroxisome proliferator-activated receptor-alpha (PPAR- $\alpha) \ldots \ldots \ldots . . .6$

Role of transcriptional factors HNF4 $\alpha$, CREB, Sp-1 .......................... 7

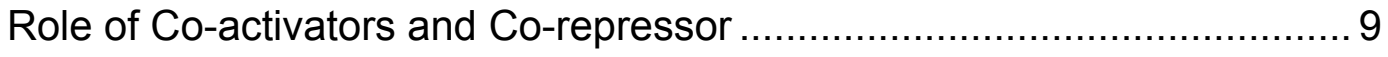

Role of Epigenetic Mechanisms in the Regulation of CPT-1 gene expression:

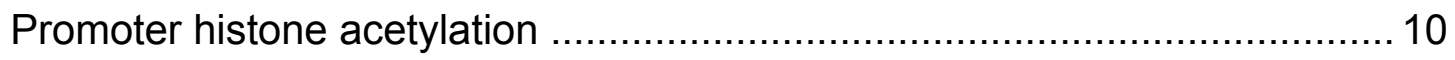

Role of Lipogenesis in Alcohol-induced hepatic steatosis ........................... 11

Alcohol-induced hepatic inflammation and injury and its Mechanisms .......... 12 
Role of kupffer cell activation by LPS in alcohol-induced liver inflammation and injury.

Role of Toll-Like receptors (TLRs) in alcohol-induced hepatic inflammation and injury. 14

Role of Monocyte Chemoattractant Protein-1 (MCP-1) and adhesion molecules in alcohol-induced hepatic inflammation and injury .................... 14 Gut-Liver Axis in Alcoholic Liver Disease ............................................... 17

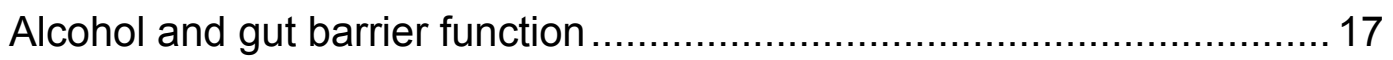

Gut-derived endotoxemia and alcohol-induced liver injury .................... 18

Role of short chain fatty acids (SCFA) specifically butyrate in intestinal

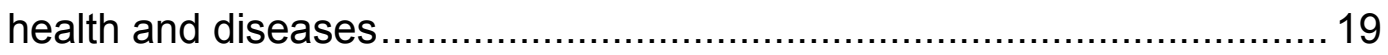

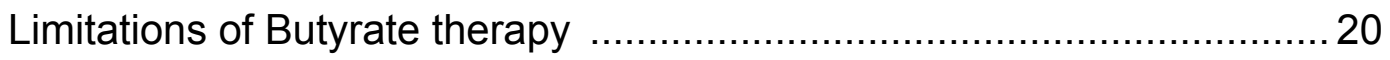

Tributyrin as a good alternative for butyrate administration .................... 21

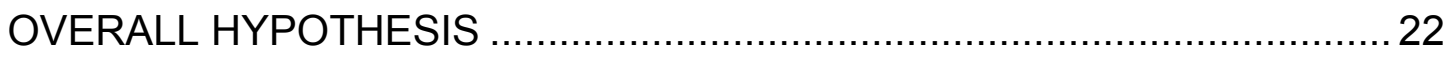

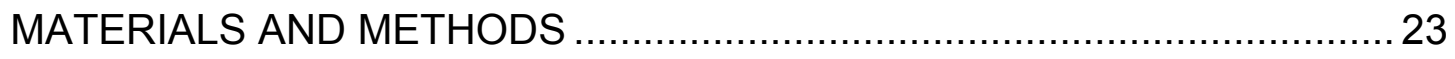

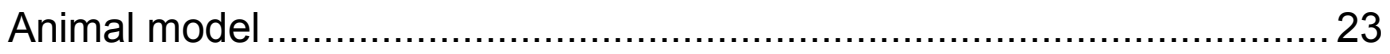

Liver Histopathological Examination ................................................. 24

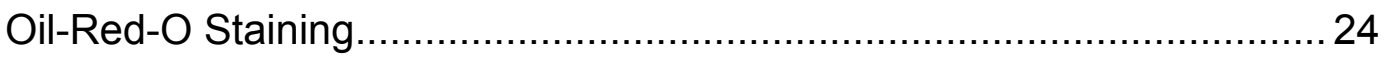

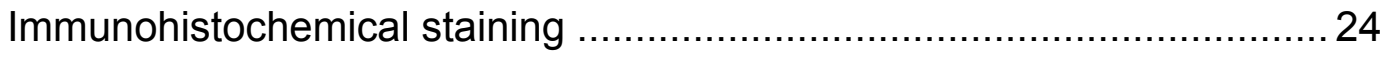

RNA Isolation and Real-Time PCR Analysis ...................................... 24

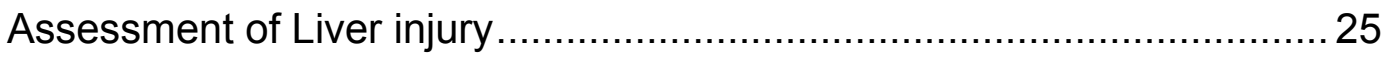

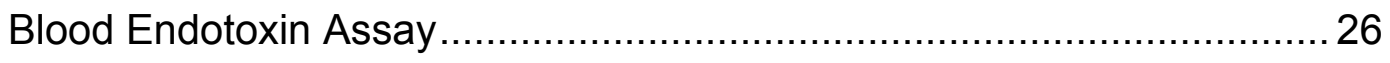

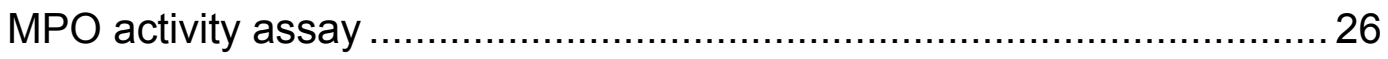




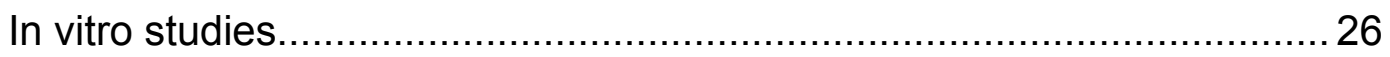

Chromatin Immunoprecipitation (ChIP) and qChIP PCR analysis........... 27

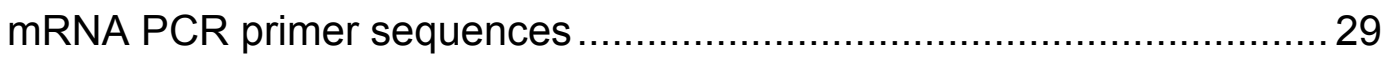

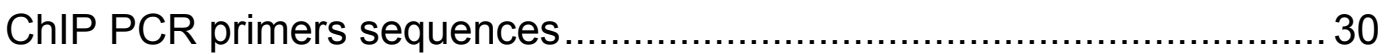

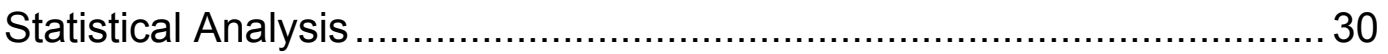

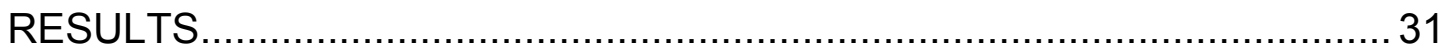

Tributyrin protects alcohol mediated down-regulation of tight-junction proteins and resultant intestinal permeability and systemic endotoxemia 31

Tributyrin attenuates alcohol-induced hepatic steatosis 35

Tributyrin administration prevents the decrease of CPT-1 gene expression in alcohol-fed mice

Evaluation of alcohol and butyrate mediated transcriptional regulation of

CPT-1 gene expression

Effect of alcohol and butyrate on PGC-1 - a key co-activator of CPT-1 gene expression

Effect of alcohol and butyrate on CPT-1 transcriptional mechanisms

Tributyrin administration protects from alcohol-induced hepatic inflammation and injury. 59

Tributyrin inhibits the alcohol-mediated macrophage activation and proinflammatory cytokine -TNF- $\alpha$ expression 59 
Butyrate down-regulated LPS inducible TNF- $\alpha$ gene expression in RAW cells 60

Tributyrin administration significantly attenuates chronic alcohol-induced MCP-1 (CCL-2) gene expression in mice and rat hepatocytes 64

Tributyrin administration significantly attenuates chronic alcohol-induced CXCL-2 (MIP-2) gene expression in mice 68

Tributryin administration significantly down-regulates adhesion molecule (ICAM-1) in alcohol-fed mice

Tributyrin administration decreases neutrophil infiltration in alcohol-fed mice 70

Administration of Tributyrin significantly attenuated Aspartate Transaminase (AST) and Alanine Transaminase (ALT) in alcohol-fed mice

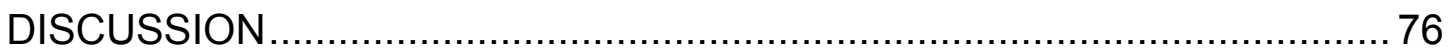

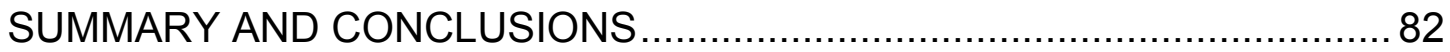

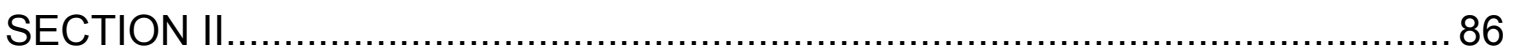

INTRODUCTION

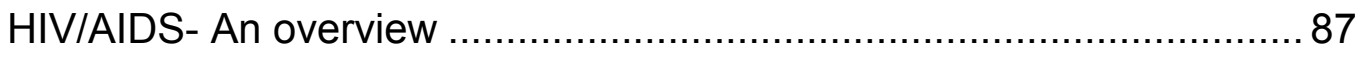

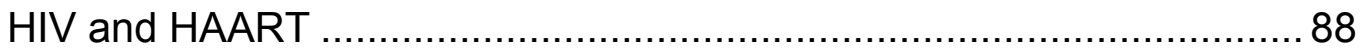

Mechanism of action of Protease inhibitors ........................................ 90

HAART associated hepatotoxicity and liver injury ................................ 91 
Role of ER-stress in HIV-PI induced liver injury ................................. 92

Role of Fas/FasL pathway in drug induced liver injury ..........................93

Role of Phosphodiesterase 4 (PDE4)/cAMP in liver injury ..................... 94

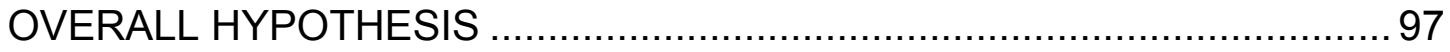

SIGNIFICANCE \& CLINICAL RELEVANCE ................................................ 98

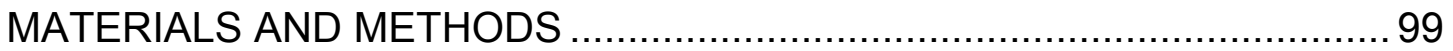

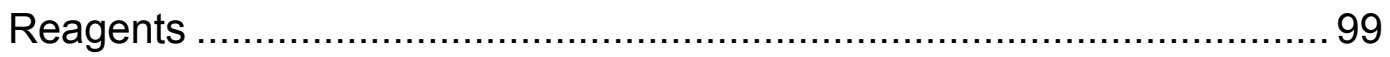

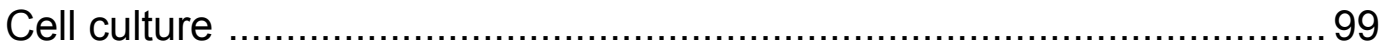

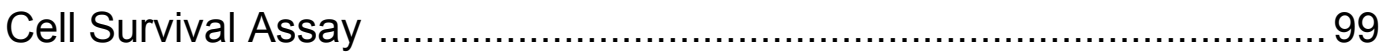

RNA Isolation and Real-Time PCR analysis ..................................... 100

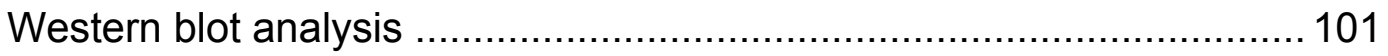

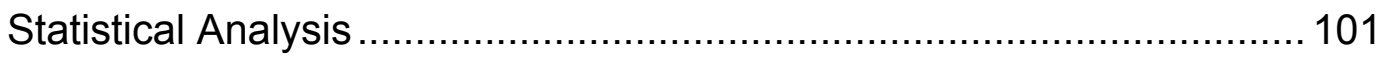

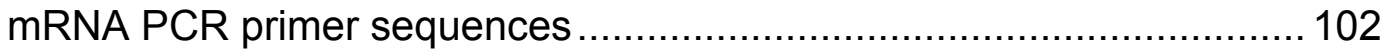

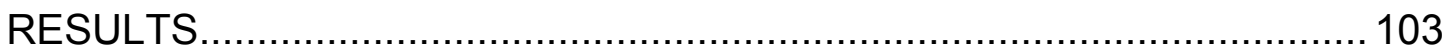

HIV-protease inhibitors induces PDE4 expression in H4IIE rat hepatoma

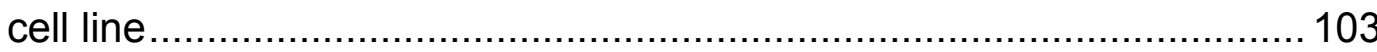

Inhibition of PDE4 led to a significant increase in the survival of HIV-PI

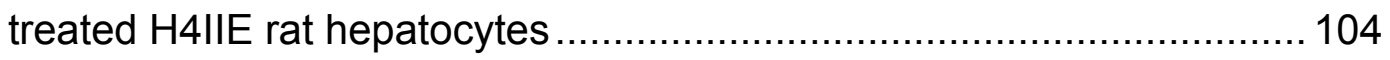

HIV-PIs induced ER-Stress was significantly attenuated by PDE4

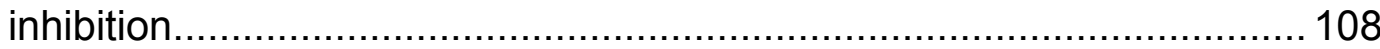

HIV-PIs induces Fas receptor and FasL gene expression in hepatocytes 109 
PDE4 inhibition down-regulates FasL expression in the hepatocytes .... 110 Role of cAMP-PKA signaling in PI induced ER-stress and FasL mediated cell death in hepatocytes 110

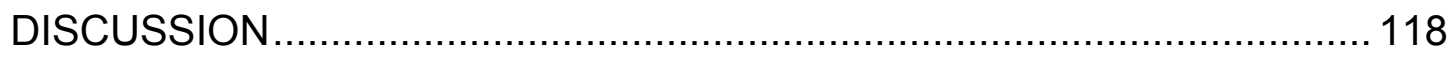

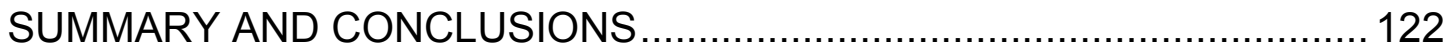

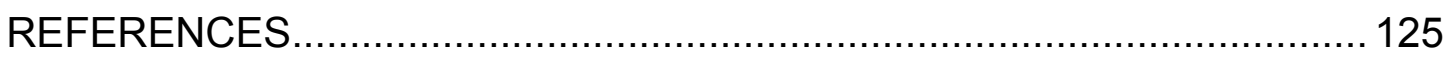

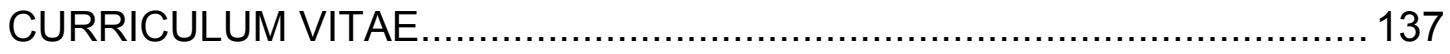




\section{LIST OF FIGURES}

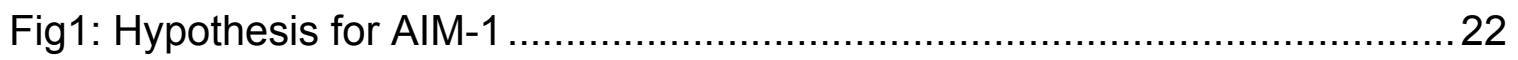

Fig2: Effect of tributyrin administration on intestinal tight junction protein expression

Fig3: Effect of tributyrin administration on alcohol-induced endotoxemia

Fig4: Effect of tributyrin administration on alcohol-induced fatty liver 36

Fig5: Effect of tributyrin administration on alcohol-induced fatty liver

Fig6: Effect of tributyrin administration on alcohol-induced hepatic steatosis as assesed by hepatic free fatty acids and triglycerides

Fig7: Effect of tributyrin administration on alcohol induced down-regulation od CPT-1 gene expression

Fig8: Effect of tributyrin administration on hepatic CPT-1 protein expression in mice liver

Fig9: Effect of butyrate on alcohol mediated down-regulation of CPT-1 gene expression

Fig10: Effect of butyrate on alcohol mediated down-regulation of PGC-1 gene expression 
Fig11: Schematic of PGC-1 promoter

Fig12: H3K9ac enhancement at PGC-1 promoter by butyrate........................4 47

Fig13: Schematic of CPT-1 promoter ....................................................... 48

Fig14: Increaser in PGC-1 binding at the CPT-1 promoter by butyrate ..............51

Fig15: Increase in p300 recruitment by PGC-1 at the CPT-1 promoter by butyrate

Fig16: Increase in H3K9ac levels at the TSS site of the CPT-1 promoter by butyrate.....

Fig17: Increase in Sp-1 binding at the Sp-1 site on the CPT-1 promoter by butyrate 56

Fig18: Increase in $\mathrm{p} 300$ recruitment by Sp-1 at the Sp-1 site on the CPT-1 promoter. .57

Fig19: Increase in RNA pol II at the TSS site on the CPT-1 promoter..... .58

Fig20: Effect of tributyrin adminstration on F4/80 positive cells in mice chronically fed alcohol for 2 weeks 61

Fig21: Chronic alcohol induced TNF- $\alpha$ gene expression 62

Fig22: LPS inducible TNF- $\alpha$ gene expression 63

Fig23: Chronic alcohol induced CCL-2 gene expression in alcohol fed mice .....66 
Fig24: Chronic alcohol induced CCL-2 gene expression in rat primary hepatocytes 67

Fig25: Chronic alcohol induced chemokine CXCL-2 gene expression 69

Fig26: Chronic alcohol induced chemokine ICAM-1 gene expression 71

Fig27: Effect of tributyrin adminintration on liver histopathology in mice chronically fed alcohol for 7 weeks 72

Fig28: Hepatic MPO activity in response to alcohol and tributyrin 73

Fig29: Effect of tributyrin administration on liver injury in mice chrnically fed alcohol for 7 weeks 75

Fig30: Hypothesis for AIM II 97

Fig31: HIV-PI drugs induce PDE4A, B abd D mRNA and protein expression ..106

Fig32: HIV-PI induces hepatocyte death is partially reversed by Rolipram 107

Fig33a: HIV-PI mediated ER-stress gene, ATF4 expression is down-regulated by

PDE4 inhibitor, Rolipram

Fig33b: HIV-PI mediated ER-stress gene, ATF3 expression is down-regulated by PDE4 inhibitor, Rolipram 113

Fig33c: HIV-PI mediated ER-stress gene, CHOP expression is down-regulated by PDE4 inhibitor, Rolipram. 114 
Fig33d: HIV-PI mediated ER-stress protein expression is significantly attenuated

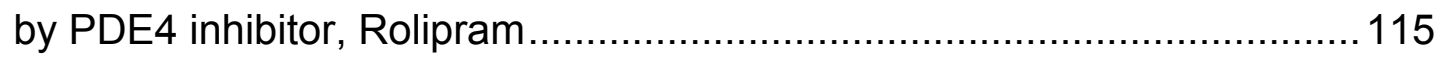

Fig34: HIV-PI mediated Fas and FasL expression is down-regulated by PDE4

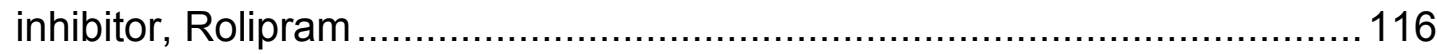

Fig35: PKA inhibitor H-89 significantly reversed the protective effect of Rolipram on ER-stress and FasL gene expression...........................................117 


\section{SECTION I}

\section{DETERMINE THE EFFECT OF TRIBUTYRIN, A DIETARY BUTYRATE PRO-DRUG ON ALCOHOL INDUCED-}
A. ALTERATIONS IN GUT-LIVER AXIS \& CONSEQUENT LIVER INJURY
B. HEPATIC CHEMOKINE EXPRESSION, LEUKOCYTE RECRUITMENT \& LIVER INJURY 


\section{INTRODUCTION}

\section{Alcoholic Liver Disease - General Overview}

Alcohol abuse exacts a major toll on health and health costs in the United States. The overall costs of alcohol abuse in the United States are estimated in some studies to be approximately $\$ 185$ billion annually. Moreover, excessive alcohol consumption is the third leading preventable cause of death in the United States. One to two million people in the U.S. are thought to have alcoholic liver disease (ALD) and approximately 14,000 people die of cirrhosis each year. In the Western world, alcohol remains the single most significant cause of liver disease, responsible for between $40-80$ percent of cases of cirrhosis. Alcoholic hepatitis is an important cause of morbidity, mortality, and health care costs in the United States and worldwide.

Clinically, the spectrum of ALD comprises of hepatic steatosis (fatty liver), steatohepatitis (hepatic inflammation) followed by fibrosis and cirrhosis sometimes leading to hepatocellular carcinoma. Amongst various stages of ALD, hepatic steatosis (fat accumulation) is an initial stage of ALD seen in $>90 \%$ of the heavy alcohol drinkers [1]. Abstinence from alcohol can lead to significant reversal of lipid accumulation in the liver. The progression from hepatic steatosis to steatohepatitis occurs in approximately $35 \%$ of the alcoholics. Pathologies such as neutrophil infiltration, kupffer cell activation, ballooning of the hepatocyte are indicative of alcohol-mediated steatohepatitis [2]. Sustained inflammation and 
steatosis further lead to fibrosis and cirrhosis. Finally, $1-5 \%$ of the cirrhotic patients develop hepatocellular carcinoma [2]. Interestingly, only $35 \%$ of the heavy drinkers develop advanced stages of ALD, which indicates that other cofactors are also involved. This includes obesity, drugs, gender, cigarette smoking and dietary factors [3-5]. Additionally, chronic alcohol consumption has synergistic effects with viruses such as Hepatitis Virus B and C as well as Human Immunodeficiency Virus.

Notably, despite decades of research on understanding the pathogenesis of liver disease there is no FDA approved therapy available to treat any form/stages of ALD. Hence it is highly relevant to study the mechanisms of the development of ALD. This would allow investigation of the potential therapeutic intervention(s) to reverse/prevent ALD and forms the basis of this dissertation proposal.

\section{Alcohol-induced Hepatic Steatosis (fatty liver) and its Mechanisms}

Steatosis is the initial, most frequent hepatic manifestation that occurs in response to acute as well as chronic ethanol consumption. Although alcoholinduced hepatic steatosis initially was considered to be a relatively benign state, it is now regarded as a significant risk factor for more progressive disease. Individuals with alcohol-induced hepatic steatosis are predisposed to develop advanced liver pathology, including alcoholic steatohepatitis (ASH), hepatic fibrosis, cirrhosis and even hepatocellular carcinoma. Hepatic steatosis is characterized by accumulation of lipid droplets (mainly triglycerides, phospholipids and cholesterol esters), which are formed by the esterification of 
free fatty acids (FFA) and glycerol in the hepatocyte cytoplasm. Alcohol-induced hepatic lipid accumulation is caused as a result of increased de novo lipogenesis, decreased fatty acid oxidation and transport of lipids via very low-density lipoprotein (VLDL) [6].

\section{Alcohol and Hepatic $\beta$-oxidation}

Alcohol consumption is known to cause disturbances in the fatty oxidation leading to excess lipid accumulation in the liver. Oxidation of fatty acids takes place in different organelles namely, mitochondria and peroxisomes specific for $\beta$-oxidation and $\omega$-oxidation [7, 8]. During fatty acid oxidation, lipids are targeted for either esterification or to mitochondrial $\beta$-oxidation. The long chain fatty acids are incorporated into the mitochondria and are oxidized by $\beta$-oxidation process. In the $\beta$-oxidation process fatty acids are transported from the cytosol to the mitochondrial matrix. These fatty acids are initially activated by coenzyme A in the cytoplasm, in the presence of fatty acyl-CoA synthetase [9]. Further, fatty acyl-CoA is then converted to fatty acyl carnitine by carnitine palmitoyl transferase (CPT-1) in the outer mitochondrial membrane. Subsequently, Carnitine translocase shuttles the fatty acyl carnitine across the inner mitochondrial membrane [9]. Finally acetyl-CoA formed in the mitochondrial matrix via beta-oxidation feeds into tricarboxylic acid cycle thus completing the oxidation to carbon dioxide and generating energy for the liver [10]. CPT-1 is the critical rate-limiting step in the $\beta$-oxidation pathway. Two isoforms of CPT-1 has been discovered [11, 12]. L-CPT-1 or liver isoform is expressed in most of the tissues including liver, kidney and pancreatic $\beta$ cells whereas M-CPT-1 or muscle 
isoform of CPT-1 is expressed in heart, skeletal muscle and adipose tissue [12, 13].

Under normal conditions, CPT- 1 controls approximately $80 \%$ of $\beta$ oxidation process in the liver and hence decrease in its expression is a crucial event in the hepatic fat accumulation [14]. Indeed, it has been shown that normalization of CPT-1 expression alone is sufficient to increase fatty acid oxidation and attenuate hepatic steatosis [15]. Specifically, these studies showed that a moderate increase in CPT-1 expression and activity is able to substantially reduce the hepatic TG burden on either a standard or high-fat diet.

Under pathologic conditions of both chronic and binge alcohol consumption, CPT-1 expression is significantly down regulated leading to hepatic steatosis [16, 17]. Importantly, increase in CPT-1 expression by resveratrol and zinc have shown to prevent chronic ethanol mediated effects $[18,19]$. Additionally, our binge alcohol feeding model has documented that prevention of ethanol induced decrease in CPT-1 expression markedly attenuates hepatic triglyceride accumulation, steatosis, and injury [17].

\section{Peroxisomal $\beta$-oxidation}

Peroxisomes are ubiquitous organelles having important metabolic functions such as $\beta$-oxidation of very long-chain $\alpha$ and $\beta$ fatty acids (VLCFA), $\alpha$ and $\beta$-oxidization of long branched chain fatty acids, synthesis of cholesterol and ether lipids[20-22]. Mechanistically, peroxisomal biogenesis occurs via de novo synthesis from the endoplasmic reticulum (ER) or via increase in number through growth and division [23-25]. Pharmacoloigcal agents such as fenofibrate, a 
peroxisome proliferator-activated receptor- $\alpha$ agonist and SCFA butyrate were shown to alleviate fatty liver by increasing the peroxisomal abundance. Although in a NAFLD mice model it has been shown that peroxisomal b-oxidation is critically involved in lipid accumulation and contributes to fatty liver disease [26], similar studies are lacking in ALD.

\section{Regulation of CPT-1 gene expression and function: Relevance to alcohol- induced hepatic steatosis \\ Role of Peroxisome proliferator-activated receptor-alpha (PPAR- $\alpha$ )}

PPAR- $\alpha$ is a nuclear hormone receptor which is abundantly expressed in the tissues such as liver and muscle that have high capacity for fatty acid oxidation such as liver and muscle [27]. Long chain fatty acids are ligands of PPAR- $\alpha$. Upon ligand binding PPAR- $\alpha$ dimerizes with retinoid $X$ receptors (RXR) at the PPAR response element (PPRE) site in the first intron of the CPT-1 gene [28]. Emerging evidence has shown that fibrates or other PPAR- $\alpha$ agonists can attenuate diet-induced fatty liver [29]. Several genes involved in transport and release of free fatty acids as well as in mitochondrial and peroxisomal fatty acid $\beta$-oxidation pathways [30] including CPT-1 are regulated by PPAR- $\alpha$ [31-33]. However, CPT-1 can also be regulated in a PPAR- $\alpha$ independent manner [34, 35].

Alcohol metabolism both in vivo and in vitro inhibits PPAR- $\alpha$ activation [36]. Previous studies by Fischer et al. [37] have shown that mice chronically fed alcohol decrease RXR- $\alpha$ protein levels reducing DNA binding of the PPAR- 
$\alpha / R X R \alpha$ heterodimer. This led to a decrease in PPAR- $\alpha$ regulated genes in the liver resulting in the development of fatty liver. Further, in these studies, a potent PPAR- $\alpha$ agonist - WY14,643 was observed to prevent the accumulation of hepatic fat in the alcohol-fed mice by increasing the PPAR- $\alpha$ expression as well as by increasing the ability of PPAR- $\alpha / R X R-\alpha$ heterodimer complex to bind to the consensus DNA response element (PPRE). Similarly, Nanji et al [38] also showed that intragastric ethanol infusion causes down-regulation of PPAR- $\alpha$ activity leading to fatty liver, which was reversed by clofibrate, a PPAR- $\alpha$ agonist. These studies document the role of PPAR- $\alpha$ in the development of alcohol induced fatty liver.

\section{Role of transcriptional factors HNF4 $\alpha$, CREB, Sp-1}

Hepatocyte nuclear factor $4 \alpha(\mathrm{HNF} 4 \alpha)$ plays a critical role in the maintenance of hepatocyte phenotype as well as in the regulation of several metabolic genes involved in gluconeogenesis, bile acids synthesis, conjugation and transport. HNF4 $\alpha$ acts as a significant transcription factor in the regulation of CPT-1 gene expression. Liver specific knockout of HNF4 $\alpha$ develop hepatomegaly and accumulation of glycogen and lipids [39, 40]. Moreover, Talianidis et al., has shown that liver specific HNF4 $\alpha$ knockout mice had $50 \%$ reduction in the CPT-1 expression[41]. In this study, recruitment of PPAR- $\alpha$ on CPT-1 promoter was independent of HNF4 $\alpha$. However the lack of induction of CPT-1 gene expression in HNF4a KO suggests that co-operation between 
activated PPAR- $\alpha$ and HNF4 $\alpha$ is essential for proper regulation of CPT-1 gene expression [41].

CREB has also been demonstrated to be a transcription factor that participates in the activation of CPT-1 gene expression [42]. CREB has been shown to bind the CPT-1 promoter region at a CAMP-responsive unit (CRU) and is comprised of two cis-acting elements - a DR1 motif and a CRE-like element (TGACG). Importantly, it has been demonstrated that both CREB and HNF4 $\alpha$ bind to the CPT-1-I promoter. Binding of both CREB and HNF4 $\alpha$ is essential for CAMP responsive CPT-1 gene expression [42]. These data also suggest that besides PPAR $\alpha, \mathrm{HNF} 4 \alpha$ can also cooperate with CREB during the transcriptional activation of CPT-1 gene expression. However the effect of alcohol on HNF4- $\alpha$ and CREB mediated transcriptional regulation of CPT-1 remains largely undetermined.

It has been shown that the transcription factors $\mathrm{Sp} 1, \mathrm{Sp} 3$ and nuclear factor $Y(N F-Y)$ bind to sites in the CPT-1 proximal promoter and participate in the developmental and hormonal control of CPT-1 transcriptional regulation as well as basal gene expression [43]. A recent study showed that plant flavonoids ingredients such as Ginkgo biloba extract up-regulate CPT-1 through increased Sp1 binding leading to enhanced fatty acid $\beta$-oxidation [44]. 


\section{Role of Co-activators and Co-repressor}

\section{Peroxisome proliferator-activated receptor- $\gamma$ coactivator (PGC-1)}

Peroxisome proliferator-activated receptor- $\gamma$ co-activator (PGC-1) act as a co-activator for several fatty acid oxidation genes, and particularly plays a critical role in the transcriptional activation [35]. The role of PGC-1 as a critical regulator of CPT-1 gene expression is clearly documented by the findings that show that overexpression of PGC-1 alone is sufficient to induce CPT-1 transcription [45]. Co-activator function of PGC-1 is activated via its deacetylation which is effected by the atypical HDAC - Sirtuin 1 (Sirt1) [46, 47]. Functionally, activated PGC-1 interacts/binds and co-activates nuclear receptor transcription factor PPAR- $\alpha$ as well as non-nuclear transcription factor HNF4 $\alpha$ during the transcriptional activation of the CPT-1 gene [41]. As a co-activator, upon binding the respective transcription factors, PGC-1 binds and recruits histone acetyltransferase (HAT) proteins including CREB-binding protein/p300 and steroid receptor coactivator-1 (SRC-1). Recruitment of HATs by PGC-1 increases promoter histone acetylation and remodeling of the chromatin that is transcriptionally permissive leading to CPT-1 gene transcription [48]

With regards to alcohol consumption Leiber's group has shown that PGC1 mRNA and protein expression were significantly down regulated during chronic alcohol feeding [49]. These data support the notion that alcohol-induced decrease in PGC-1 expression is a major pathogenic mechanism that contributes to the decrease in CPT-1 gene expression and development of hepatic steatosis. 


\section{Role of Epigenetic Mechanisms in the Regulation of CPT-1 gene expression Promoter histone acetylation}

Gene expression is dynamically regulated by chromatin modifications on histone tails, and histone acetylation promotes transcription, whereas histone deacetylation negatively regulates transcription. Steady-state levels of acetylation result from the balance between the opposing activities of histone acetyltransferases (HATs) and HDACs. Transcriptional activation of CPT-1 has been demonstrated to be influenced by both HATs as well as HDACs [17]. HATs - particularly, CBP/p300 and SRC- 1 can be recruited to the CPT-1 gene by PGC-1 co-activator that increases promoter histone acetylation and transcriptional activation [50] In contrast, HDACs - particularly HDAC3 can also be recruited to the CPT-1 promoter by the co-repressor $\mathrm{N}-\mathrm{CoR}$ that decreases promoter histone acetylation and transcriptional activation [50]. Work done by our group has demonstrated that increase in HDAC3 expression and consequent $\mathrm{N}-\mathrm{CoR}-\mathrm{HDAC} 3$ mediated down-regulation of CPT-1 expression is the key epigenetic mechanism that underlies binge alcohol induced hepatic steatosis and injury [17]. Moreover, the data obtained from this work also demonstrated that by targeting HDAC3 activity, the binge alcohol-induced decrease in CPT-1 can be prevented and hepatic steatosis markedly attenuated [17]. Importantly, these data also strongly implicated that targeting HDAC activity represents a potential therapeutic approach in the treatment of binge alcohol-induced hepatic steatosis 


\section{Role of Lipogenesis in Alcohol-induced hepatic steatosis \\ Sterol regulatory element-binding proteins (SREBPs)}

Another mechanism that underlies the development of hepatic steatosis is lipogenesis. Majority of the enzymes and proteins involved in lipogenesis caused by alcohol are regulated by SREBPs. When activated, SPREBPs that are bound to the endoplasmic reticulum are released into the nucleus. SREBPs plays a critical role in regulating the synthesis of cholesterol, fatty acids and triglycerides in liver by modulating the activation of more than 30 genes in the liver including fatty acid synthase, ACC, stearoyl-CoA desaturase, ATP citrate lyase, and malic enzyme. Several studies have shown the involvement of SREBP-1 in the progression of fat accumulation using in vivo as well as in vitro systems. [51] During alcohol feeding increased levels of SREBP-1 protein and mRNA was observed in alcohol-fed mice [52]. Metabolism of alcohol to acetaldehyde increases the formation of mature SREBP-1 protein enhancing fat production (hepatic lipogenesis) leading to fatty liver.

\section{Mammalian sirtuin-1 (SIRT-1)}

SIRT1, a NAD ${ }^{+}$- dependent class III histone deacetylase protein is a metabolic master regulator. SIRT-1 plays an important role in regulating lipid metabolism and inflammation by targeting the acetylation status of various targets such as histones, transcriptional regulators and their co-activators [5355]. Alcohol consumption down-regulates SIRT-1 in hepatic cells and tissues leading to excess fat accumulation and inflammatory responses which progresses to alcoholic fatty liver disease (AFLD) [49, 56-58]. SIRT-1 has been 
shown to bind to Sterol regulatory element-binding proteins (SPREBP-1) resulting in its inactivation via deacetylation. Alcohol consumption reduces the level of SIRT-1 resulting in the higher levels of acetylated nuclear form of SREBP-1c causing increased fat production in the liver. Acute and chronic alcohol consumption increases the expression of several SREBP-1 regulated fatty acid biogenesis related enzymes leading to excess hepatic lipid accumulation. Importantly, SREBP-1 KO mice were significantly protected from AFLD [59]. SIRT1 also de-acetylates PGC-1 in at least 13 lysines at different domains of the protein[60]. SIRT1 directly interacts with and maintains PGC-1 in the deacetylated active form bound to the chromatin thus increasing the rate of transcription of CPT-1 $[46,47]$. This indicates that PGC-1 could undergo cycles of acetylation and deacetylation that are SIRT1 activity dependent [60]. Additionally, SIRT-1 positively regulates PPAR- $\alpha$. As mentioned before, PGC-1 co-activates with PPAR- $\alpha$ to induce mitochondrial fatty acid oxidation enzymes. Hence disruptions in the regulation of PPAR- $\alpha$ and PGC-1 due to downregulation of SIRT-1 have been implicated in the development of AFLD in animals [61-64].

\section{Alcohol-induced hepatic inflammation and injury and its Mechanisms}

A major factor responsible for the development of alcoholic liver disease is immune dysregulation and increased production of pro-inflammatory cytokines/chemokines which leads to chronic hepatic inflammation. Liver inflammation is associated with hepatomegaly as well as increased infiltration of granulocytes, monocytes and lymphocytes. 


\section{Role of kupffer cell activation by LPS in alcohol-induced liver inflammation and injury}

Several reports have shown that chronic alcohol abuse leads to an increase in gram-negative bacteria in the intestine. Additionally, alcohol is known to disrupt the gut barrier function by decreasing tight junction protein expression in the intestine and causing endotoxemia by lipopolysaccharide (LPS) release in the systemic circulation $[65,66]$. Endotoxin is recognized by hepatic macrophages (kupffer cells) in the portal circulation and is removed from the systemic circulation. In the liver, exposure of LPS to kupffer cells results in kupffer cell activation leading to the production of inflammatory cytokines such as TNF- $\alpha$ and reactive oxygen species (ROS). These inflammatory cytokines and ROS further activate other liver cells such as hepatocytes, stellate cells and endothelial cells and enhance LPS induced liver damage[67]. Our lab and several studies have shown that LPS inducible TNF- $\alpha$ production is increased in chronic alcoholics and in alcohol-fed animals $[68,69]$. Role of TNF- $\alpha$ in alcoholic liver disease is further documented by McClain et al. where elevated serum levels of TNF- $\alpha$ inducible cytokines and chemokines including IL-6, IL-8 and IL18 were reported in alcoholic hepatitis $(\mathrm{AH})$ patients [70]. Notably, alcohol administration to TNF- $\alpha$ knockout mice did not develop liver injury [71], confirming the pathogenic role of inflammation in the development of ALD. 
Role of Toll-Like receptors (TLRs) in alcohol-induced hepatic inflammation and injury

TLRs are pattern recognizing receptors that sense pathogen-derived molecules as well as host-derived danger signals $[72,73]$. TLRs are widely expressed on hepatocytes as well as kupffer cells. Out of 10 different TLRs, TLR4 is known to be highly relevant in ALD and is widely studied. LPS binds to TLR4 and activates the production of inflammatory cytokines suggesting that TLRs play a critical role in the pathophysiology of various liver disease [74]. LPS/TLR4 signaling pathway activates transcription factors such as NF-kB that induces pro-inflammatory cytokines such as IL-6, IL-8 and TNF- $\alpha$ leading to liver injury $[75,76]$. Exposure to LPS during alcohol consumption results in upregulation of TLR-4 expression on the Kupffer cells as well as other cell types in the liver. This LPS/TLR4 signaling plays an important role in the pathogenesis of ALD [77].

\section{Role of Monocyte Chemoattractant Protein-1 (MCP-1) and adhesion molecules in alcohol-induced hepatic inflammation and injury}

In addition to the role of TLR4/LPS signaling pathway and TNFproduction, chemokines and adhesion molecules are also equally responsible for the development of chronic alcohol induced liver injury [78, 79]. Neutrophil infiltration and monocytes recruitment in the liver is associated with chronicalcohol induced liver injury. Chemokine such as CCL2 also referred to as monocyte chemotactic peptide-1 (MCP-1), is a member of the C-C chemokine family. It is expressed in most of the liver cell types such as hepatocytes, stellate 
cells and kupffer cells $[80,81]$ and is a potent chemotactic as well as chemoattractant for monocytes $[82,83]$. Previous studies have shown that CCL2 mRNA and protein expression is up regulated in chronic alcohol feeding in rodents as well as in alcoholic hepatitis patients [80, 84-88]. Increased levels of CCL2 recruited and activated monocytes /macrophages to the damaged tissue site and stimulated pro-inflammatory cytokines production such as TNF- $\alpha$, IL-1 $\beta$ and IL-6 $[89,90]$. Apart from activating macrophages, CCL2 plays a major role in the development of hepatic steatosis and liver injury. Studies in primary hepatocytes have demonstrated that CCL2 induces lipid accumulation during tissue injury [91]. Moreover, a transgenic mouse overexpressing CCL2 in adipose tissue showed increased hepatic triglycerides levels [92]. Interestingly, CCL2 knockout mice were protected against alcoholic liver injury by the induction of genes responsible for fatty acid oxidation and inhibition of pro-inflammatory cytokines [93]. Since CCL2 regulates macrophage activation, pro-inflammatory responses and hepatic steatosis in a chronic alcohol mice model, pharmacological approaches to block CCL2 would be beneficial in treating early stages of alcoholic liver disease such as steatohepatitis. Intercellular adhesion molecule (ICAM-1) is expressed on kupffer cells, hepatocyte and endothelial cells. ICAM-1 recruits leukocytes into inflammatory sites by binding to its ligands LFA-1 or Mac-1, which are members of the leukocyte integrin family. The activated leukocytes injure tissue by releasing oxidants and proteases. Thus ICAM-1 could be involved in the pathogenesis of inflammatory liver diseases such as ALD. ICAM-1 is associated with chronic 
alcohol exposure in patients with alcoholic liver disease [79]. Previous studies done by Kono group have shown that ICAM-1 knockout mice were protected from chronic ethanol induced liver injury by decreasing neutrophil infiltration and hepatic steatosis [78]. Hence ICAM-1 plays a critical role in the recruitment of neutrophils in alcohol-induced liver injury. 


\section{Gut-Liver Axis in Alcoholic Liver Disease}

\section{Alcohol and gut barrier function}

There is increasing evidence that demonstrates a critical pathogenic role for the gut-liver axis in the development of ALD [94]. Alcohol has been shown to change the composition of the microbiome as well as impair intestinal integrity and barrier function [94, 95]. Integrity of intestinal barrier function is regulated, in part, by the epithelial tight junction (TJ) complex composed of proteins such as zonula occludens (ZO), occludin and claudins [96, 97]. The TJ complex establishes intestinal barrier integrity by connecting the inter-epithelial cell spaces and inhibiting the paracellular passage of microbes and microbial products and other luminal contents [98-100]. An intact intestinal epithelial barrier plays a major role in preventing intestinal invasion of luminal pathogens and antigenic molecules and their subsequent migration to the liver and potentially the systemic immune system. Accordingly, portal and systemic bacterial derived lipopolysaccharide (LPS) levels are nearly undetectable in normal rats as well as healthy people $[76,101,102]$. The clinical relevance of intestinal barrier dysfunction has been recognized by its pathogenic association with the development of ALD [103]. Direct evidence for decreased gut barrier function and increased intestinal permeability was provided by the observations that oral administration of LPS to alcohol-fed rats significantly increased systemic endotoxin levels [104]. 


\section{Gut-derived endotoxemia and alcohol-induced liver injury}

Besides the disruption of the gut barrier function, alcohol induced alterations in the gut microbiota (dysbiosis) also can cause increased intestinal endotoxin production, enhanced metabolism of ethanol into acetaldehyde leading to further increase in intestinal permeability and translocation of bacteria and bacterial products [105-112]. Recently our group had performed metagenomic analyses to examine the effects of chronic alcohol feeding on the gut bacterial microbiome by employing deep 16s rRNA gene sequencing using DNA isolated from fecal samples [109]. The findings from this study showed that alcohol feeding leads to significant shifts in the gut bacterial community with a marked increase in the phylum Proteobacteria comprised of gram negative bacteria that contain LPS in their cell wall and are the principal source of intestinal endotoxin and systemic endotoxemia. Importantly, ethanol-mediated endotoxemia, along with hepatic steatosis, inflammation and injury were markedly attenuated by treatment with a probiotic Lactobacillus rhamnosus GG (LGG).

Due to its anatomical links to the gut, the liver is constantly exposed to gut-derived bacterial products, and functions as a major filter organ and a first line of defense. The frequency of systemic endotoxemia has been observed to be significantly greater in patients with alcoholic cirrhosis than in non-alcoholic cirrhotic subjects $[76,113]$. Notably, plasma endotoxin levels are also significantly elevated in alcohol consuming subjects without evidence of liver disease [113]. These findings suggest a direct role for alcohol in inducing systemic endotoxemia. Gut-derived endotoxin is a crucial mediator of liver injury 
and hepatic fibrosis/cirrhosis [114-116]. Importantly, gut-derived endotoxin, seems to play a major role in the pathogenesis of ALD, as demonstrated by the reduction of alcoholic liver injury following elimination of the Gram-negative microbiota by antibiotics [117] and the sensitization to LPS-induced liver injury following long-term ethanol exposure [118, 119].

\section{Role of short chain fatty acids (SCFA) specifically butyrate in intestinal health and diseases}

The human gut harbors large and dynamic bacterial community consisting of 300-500 different species of bacteria and the number of microbial cells within the gut is 10 times larger than the eukaryotic cells in the human body [120,121]. Short chain fatty acids are the processed by-products of the undigested long chain polysaccharide by anaerobic bacteria present in the gut. The short chain fatty acids found in the intestinal lumen includes acetate, propionate and butyrate $[122,123]$ in the constant molar ratio of $60: 25: 15$. Although butyrate is the least abundant SCFA, it is important in maintaining the colonic mucosal health and also serves as a main energy source for colonocytes [124]. In the colon grampositive anaerobic bacteria produce butyrate. Specifically, two groups of butyrate-producing bacteria are Faecalibacterium prausnitzii. belonging to the Clostridium leptum cluster, and Eubacterium rectale/Roseburiaspp. belonging to the Clostridium coccoides cluster of firmicute bacteria [125]. During the fermentation process decrease in the luminal $\mathrm{pH}$ is conducive for the growth of butyrate-producing bacteria [126]. Previous studies from our lab have shown that chronic alcohol administration alters the intestinal microbial environment by increasing luminal $\mathrm{pH}$ leading to hepatic steatosis and inflammation and injury 
[109]. This could possibly explain the butyrate depletion in chronic alcohol consumption.

Butyrate enters the cell by non-ionic diffusion, $\mathrm{SCFA} / \mathrm{HCO}_{3}^{-}$exchange, and active transport by SCFA transporters. Studies have shown that butyrate exhibits a protective role by acting as a HDAC inhibitor [127]. Butyrate inhibits majority of the HDACs except class II HDAC-6 and -10 and class III [127]. In colon carcinoma cells, butyrate causes an imbalance in the histone acetylation state affecting transcription of genes involved in cell cycle differentiation and apoptosis and progression of cancer.[127-131]. Additionally, butyrate has been shown to inhibit inflammatory responses through NFKB inhibition [132-134]. Butyrate also has a potential application in treating and preventing molecular syndromes such as diet induced obesity and insulin resistance in humans [135]. Overall butyrate has a wide spectrum therapeutic potential in treating many disease state as well as maintaining intestinal homeostasis by different mechanisms the most common being butyrate acting as an HDAC inhibitor.

\section{Limitations of Butyrate therapy}

Although previous literature data has shown that butyrate has wide spectrum possibilities as a therapeutic potential there are certain limitation to its administration. Parental administration of butyrate has a very short plasma halflife due to which a sustained infusion of butyrate via i.v dosing is needed to maintain the therapeutic concentration of butyrate in the plasma [136-139]. High doses of i.v. butyrate solution can cause hypernatremia with narrow safety window [140]. 


\section{Tributyrin as a best alternative for butyrate administration}

Tributyrin (a prodrug of butyrate) is a triglyceride containing three butyrate moieties esterified to glycerol. It is found in variety of foodstuff such as dairy products [141]. Tributryin has better pharmacokinetic and pharmacodynamics properties as compared to butyrate. The half-life of tributyrin is $40 \mathrm{mins}$ and it is well tolerated when compared to i.v. butyrate solution [140, 142]. Oral administration of Tributryin exhibits direct effects on liver by increasing the hepato-portal concentration of butyrate $[142,143]$. Recent research by Miyoshi has shown that oral administration of tributyrin, upon digestion in the upper $\mathrm{GI}$ tract, increases the butyrate concentration in the hepato-portal circulation and attenuates LPS-induced liver injury and inflammatory reactions via suppression of NF-kB activation. 


\section{OVERALL HYPOTHESIS}

We hypothesized that oral administration of tributyrin significantly attenuates alcohol-induced hepatic steatosis, inflammation and injury in mice (Fig.1)

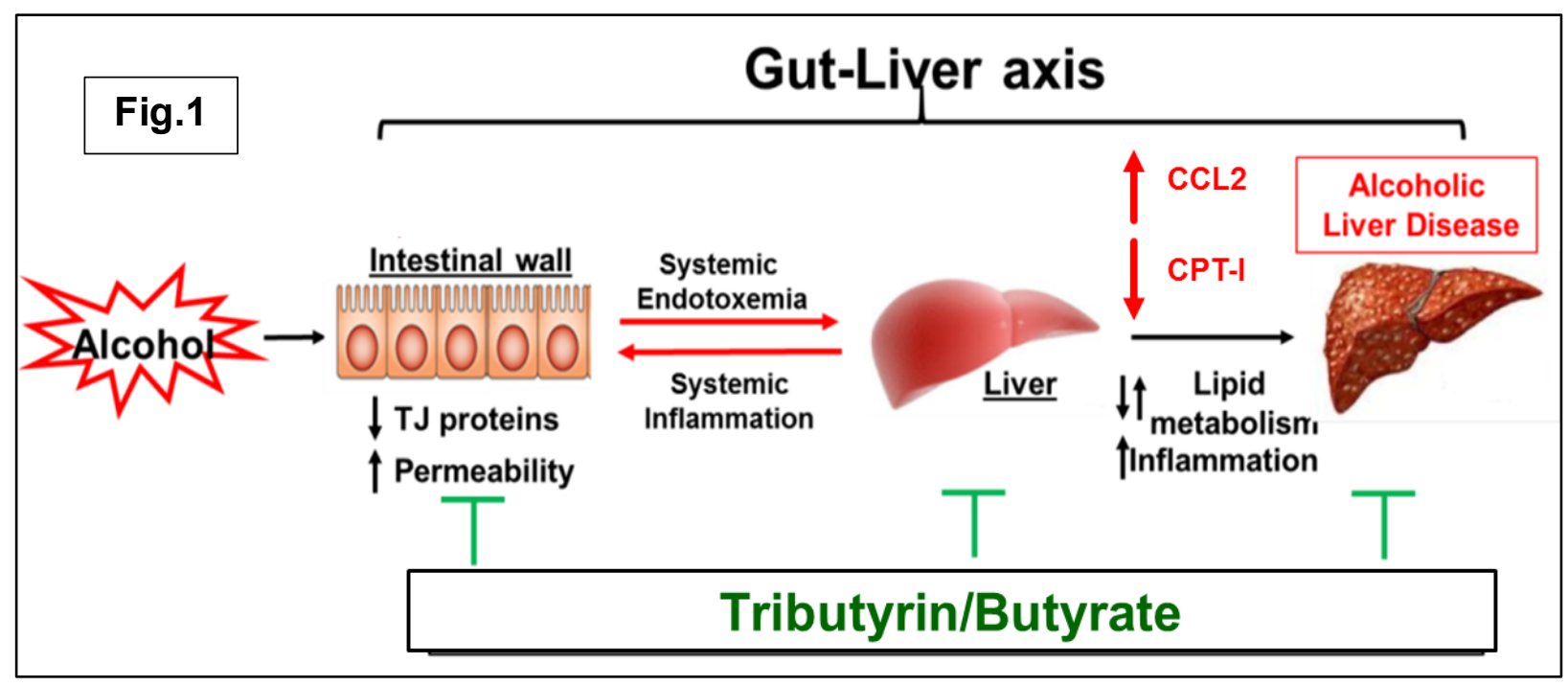




\section{MATERIALS AND METHODS}

\section{Animal Model}

Eight-week-old male C57BL/6N mice were obtained from Harlan (Indianapolis, IN). All mice were housed in a pathogen-free, temperature-controlled animal facility accredited by the Association for Assessment and Accreditation of Laboratory Animal Care with 12 hour light/12 hour dark cycles. All experiments were carried out according to the criteria outlined in the Guide for Care and Use of Laboratory Animals and with approval of the University of Louisville Animal Care and Use Committee. Animals were fed a modified Lieber-DeCarli liquid diet containing alcohol (35\% of total calories) and enriched in unsaturated fat (corn oil) Research Diet, New Brunswick, NJ. Control liquid maltose-dextrin diets provided $40 \%$ of energy from fat, $43 \%$ - from carbohydrate, $17 \%$ - from protein. Initially, all mice were given the control liquid maltose dextrin diets ad libitum for one week. Afterwards, mice were fed either the liquid ethanol diet or the control liquid maltose-dextrin diet. Ethanol was gradually increased every 3-4 days from $11.2 \%$ to $35 \%$ of total calories $(5.0 \%$ [vol $/ \mathrm{vol}])$. The mice were fed with the alcohol diet ( $5 \%$ alcohol vol $/ \mathrm{vol})$ ad libitum. The control mice were pair-fed with maltose-dextrin diets on an isocaloric basis. Tributyrin (Tb) purchased from Sigma Aldrich (St. Louis MO) was orally gavaged at $2 \mathrm{~g} / \mathrm{kg} 5$ days a week in control mice as well as alcohol-fed mice. 


\section{Materials}

Tributyrin was purchased from Sigma (St. Louis MO)

\section{Liver Histopathological Examination}

For histological analysis, liver sections were fixed in $10 \%$ buffered formalin for 24 hours and embedded in paraffin. Tissue sections were stained with hematoxylineosin (H\&E) and examined under light microscopy at 40x magnification.

\section{Oil-Red-O Staining}

To examine the amount of fat accumulation, the liver sections were stained with Oil-Red-O. Frozen liver sections were washed in phosphate buffered saline twice for 5 minutes. Oil-Red-O and $85 \%$ propylene glycol were added with agitation for 15 minutes, followed by washing in tap water.

\section{Immunohistochemical staining}

Mice liver sections were stained with $\mathrm{F} 4 / 80+$, and CAE staining, and tightjunction proteins such as anti-ZO-1 and Claudin antibody. Commercially available antibody against and CPT-1 (Proteintech Group Inc., Chicago, IL) was used for CPT-1 analysis. Assays were performed according to the manufacturers' protocols.

\section{RNA Isolation and Real-Time PCR Analysis}

Total RNA was isolated from the liver tissues using TRIzol®, according to manufacturer's instructions. For real time PCR, the first strand cDNA was synthesized using qScriptTM cDNA SuperMix (QuantaBiosciences, Inc, Gaithersburg, MD) using 400ng of the total RNA. The RT conditions were 10 
mins at $25^{\circ} \mathrm{C}, 30$ mins at $48^{\circ} \mathrm{C}$ and 5 mins at $85^{\circ} \mathrm{C}$. Real time PCR was performed with an $A B I$ prism 7500 sequence detection system and PerfeCtaTM SYBR Green FastMixTM, Low ROX reagent (Quanta Biosciences, Inc.).Each sample was run in duplicate reactions. Reverse transcriptase polymerase chain reaction (RT-PCR) assays were used to assess mRNA levels of desired genes liver tissues and in primary hepatocytes. The primers amplifying the genes examined were designed in the lab and obtained from Intergrated DNA Technologies, Inc (Coralville, IA). The parameter threshold cycle (Ct) was defined as the fraction cycle number at which the fluorescence passed the threshold. The relative gene expression was analyzed using the 2-AACt method by normalizing with $\beta$-actin mRNA gene expression in all the experiments and is presented as fold change over Untreated which is set at 1.

\section{Assessment of Liver injury}

Kits for biochemical analysis were purchased from the following: alanine aminotransferase (ALT), Aspartate Aminotransferase (AST) and triacylglycerols (TAG), Thermo Electron (Melbourne, Australia); nonesterified-fatty acid (NEFA), Wako Chemical USA (Richmond, VA, USA). For liver TAG assay, hepatic tissue (100 mg) was homogenized in $50 \mathrm{mM} \mathrm{NaCl}$. The homogenate $(500 \mu \mathrm{l})$ was mixed with chloroform/methanol (2:1, $4 \mathrm{ml})$ and incubated overnight at room temperature with gentle shaking. Homogenates were vortexed and centrifuged for 5 min at $3000 \times \mathrm{g}$. The lower lipid phase was collected and concentrated by vacuum. The lipid pellets were dissolved in 1\% Triton X100 in phosphate- 
buffered saline, and hepatic TAG content was determined via enzymatic colorimetric methods.

\section{Blood Endotoxin Assay}

Plasma endotoxin levels were measured with Limulus Amebocyte Lysate kit (Lonza, Walkerville, MD) according to manufacturer's instructions.

\section{MPO activity assay}

For hepatic myeloperoxidase activity (MPO), liver samples were homogenized (10ug/200ul) in 200mM Nacl, 5mM EDTA, 10mM tris, 10\% glycerin and centrifuge at $1500 \mathrm{~g}$ for $15 \mathrm{mins}$. Activity was measured according to manufacturer's instuctions (Hycult Biotech Plymouth Meeting, PA) at 450nm using a plate reader. Results are expressed as mg of MPO activity/ gram of protein, as determined by Lowry Protein assay (Bio-Rad Hercules, CA)

\section{Invitro studies}

Rat primary hepatocytes were used in this study. Hepatocytes were isolated from sprague dawley rats. Hepatocytes were treated with alcohol $(50 \mathrm{mM})$ with/without $\mathrm{NaB}(2 \mathrm{mM})$ which was pre-treated for half hour. At the end of 48 hours, alcohol and/or butyrate treated cells were treated with/without TNF- $\alpha(20 \mathrm{ng} / \mathrm{ml})$. RAW 267.4 macrophages were also used in this study. They were treated with LPS $(10 \mathrm{ng} / \mathrm{ml})$ for 4 hours and pre-treated with/without $\mathrm{NaB} 2 \mathrm{mM}$ and Triochostain A (TSA) $(100 \mathrm{ng} / \mathrm{ml})$ for half hour. All cells were cultured in Dulbecco's modified eagle medium (DMEM) supplemented with $10 \%$ fetal bovine serum (FBS), $10 \mathrm{U} / \mathrm{ml}$ penicillin, and 10 g/ml streptomycin. Cells were 
maintained in an incubator at $37^{\circ} \mathrm{C}$ with humidified $5 \% \mathrm{CO} 2$. Cells were plated and incubated overnight before treatment, in all experiments.

\section{Chromatin Immunoprecipitation (ChIP) and qChIP PCR analysis}

The ChIP assay was conducted using the ChIP assay protocol established in the lab. Briefly, $10 \times 10^{6}$ cells were crosslinked with $1 \%$ formaldehyde- $1 \mathrm{X}$-glycine and lysed in lysis buffer containing protease inhibitor cocktail. Enzymatic digestion of DNA was performed to produce chromatin fragments of 100-166 bp, which were confirmed by running the chromatin on a $1 \%$ agarose gel. ChIP antibodies directed against protein of choice were used for immunoprecipitation. Anti-acetyl H3K9, anti-p300, anti-PGC-1, anti-Sp-1 and anti-RNA Polymerase II were from EMDMillipore (MA, USA). The lysates were incubated overnight with antibody, following which the antibody/protein complexes were immunoprecipated using magnetic beads. Successive washes were performed with low salt, high salt, $\mathrm{LiCl}$ and Tris EDTA (TE) wash buffers. After reverse crosslinking and purification, pure DNA was eluted in elution buffer (1\% SDS, 0.1 M NaHCO3). qChIP-PCR was performed using the ABI Prism 7500 Sequence Detection System. ChIP- PCR primers designed for the regions of the CPT-1 and PGC-1 promoters were used and their sequences are as follows:

Data was analyzed as Differential Occupancy Fold Change. ChIP-qPCR results were calculated by $\Delta \Delta \mathrm{Ct}$ method (since all Ct values for a binding site are determined from the same qPCR assay) where each ChIP DNA fractions Ct value was normalized to the Input DNA fraction Ct value using $\Delta \mathrm{Ct}$ [normalized $\mathrm{ChIP}]=(\mathrm{Ct}[\mathrm{ChIP}]-(\mathrm{Ct}[$ Input $])$. The difference between the normalized 
experimental sample (S2) and the control sample (S1) ChIP fraction Ct values (second $\Delta \Delta \mathrm{Ct}$ ) was determined using $\Delta \Delta \mathrm{Ct}$ [S2-S1] $=\Delta \mathrm{Ct}$ [S2: normalized ChIP] $-\Delta \mathrm{Ct}[\mathrm{S} 1:$ normalized ChIP]. 
mRNA PCR primer sequences

\begin{tabular}{|l|l|}
\hline Rat & \\
\hline r.n_CPT-1a_F2 & CTGCATGGAAGATGCTTTGA \\
\hline r.n_CPT-1a_R2 & GCCATGACATACTCCCACAA \\
\hline r.n._Ccl2_F2 & \\
\hline r.n._Ccl2_R2 & GTCTCAGCCAGATGCAGTT \\
\hline Mouse & CTCTTGAGCTTGGTGACAAAT \\
\hline mm_CPT-1a_F1 & \\
\hline mm_CPT-1a_R1 & GCTGCACTCCTGGAAGAAGA \\
\hline & \\
\hline mmCcl2_F & GGCTCAGCCAGATGCAGT \\
\hline mmCcl2_R & TGAGCTTGGTGACAAAAACTACAG \\
\hline & \\
\hline mmCxcl2_F3 & GCGCCCAGACAGAAGTCATA \\
\hline mmCxcl2_R3 & TCCAGGTCAGTTAGCCTTGC \\
\hline & \\
\hline mm_Icam1_F2 & CGCTGTGCTTTGAGAACTGT \\
\hline mm_Icam1_R2 & TGAGGTCCTTGCCTACTTGC \\
\hline & \\
\hline m_Actb_F2 & CAGCTGAGAGGGAAATCGTG \\
\hline m_Actb_R2 & CTCCAGGGAGGAAGAGGATG \\
\hline & \\
\hline m_Tnf_F2 & GTGATCGGTCCCCAAAGG \\
\hline rod_Tnf_R2 & GGTGGTTTGCTACGACGTG \\
\hline
\end{tabular}




\section{ChIP PCR Primers}

\begin{tabular}{|l|l|}
\hline CPT-1 TRE For & GGTGACGTTGGCTGAGCAA \\
\hline CPT-1 TRE Rev & TGAGCCCCTGTACACGTTTTG \\
\hline & \\
\hline r.n.CPT-1_Chp2_F & ATGGGCATGGCTTTAATGAG \\
\hline r.n.CPT-1_Chp2_R & GGCTAGGACCCGAGCTTGT \\
\hline & \\
\hline & \\
\hline r_n_CPT-1GCTSs_F4 & AGCCTCGCCCGCCCCTGCTC \\
\hline r_n_CPT-1_GCTSs_R4 & CAGCGCTGCCCTCCCGGTGTC \\
\hline & \\
\hline mm_Ppargc1a_TSS_F4 & TGACAGCCCAGCCTACTTTT \\
\hline mm_Ppargc1a_TSS_R4 & CACACAGCACACACTCATGC \\
\hline
\end{tabular}

\section{Statistical Analysis}

Data are presented as means \pm SD for the indicated number of independently performed experiments. Student's t-test and one-way ANOVA with Bonferroni multiple comparison test were used for the determination of statistical significance. $P<0.05$ was considered significant. 


\section{RESULTS}

\section{Tributyrin protects alcohol mediated down-regulation of tight-junction}

proteins and resultant intestinal permeability and systemic endotoxemia Intestinal tight junctions $(\mathrm{TJ})$ play a major role in maintaining the barrier integrity, thus preventing release of toxins such as endotoxin into the systemic circulation. ZO-1 and Occludin are tight junction proteins, critical for the gut integrity and barrier function. Initially we investigated the effect of tributyrin on TJ proteins in chronic alcohol fed animals. Mice were orally administered tributyrin at a concentration of $2 \mathrm{~g} / \mathrm{kg}$ with or without $5 \%$ Alcohol via Lieber DeCarli diet for 4 weeks. The expression of TJ proteins specifically ZO-1 and Occludin was examined by immunostaining the intestinal ileum sections (Fig. 2). Similar to other studies, we also observed that chronic alcohol administration decreased ZO-1 and Occludin protein expression in the ileum compared to pair-fed mice. Notably, tributyrin administration prevented the chronic alcohol-induced loss of ZO-1 and occludin proteins. Tributyrin alone group showed no change in expression of these proteins. This data showed that tributyrin administration can restore the barrier integrity by preventing the alcohol-induced decrease in tight junction proteins. Hence, we next examined the effect of tributyrin on barrier function/permeability by measuring the systemic endotoxin levels. The data showed that chronic alcohol consumption caused increase in systemic endotoxin levels. Importantly, tributyrin treatment significantly attenuated alcohol-induced 
increase in serum endotoxin levels (Fig.3). Overall, these data showed that tributyrin protects the gut barrier function by restoring tight-junction proteins and resultant decrease in intestinal permeability and systemic endotoxemia, observed in chronic alcohol fed animals. 


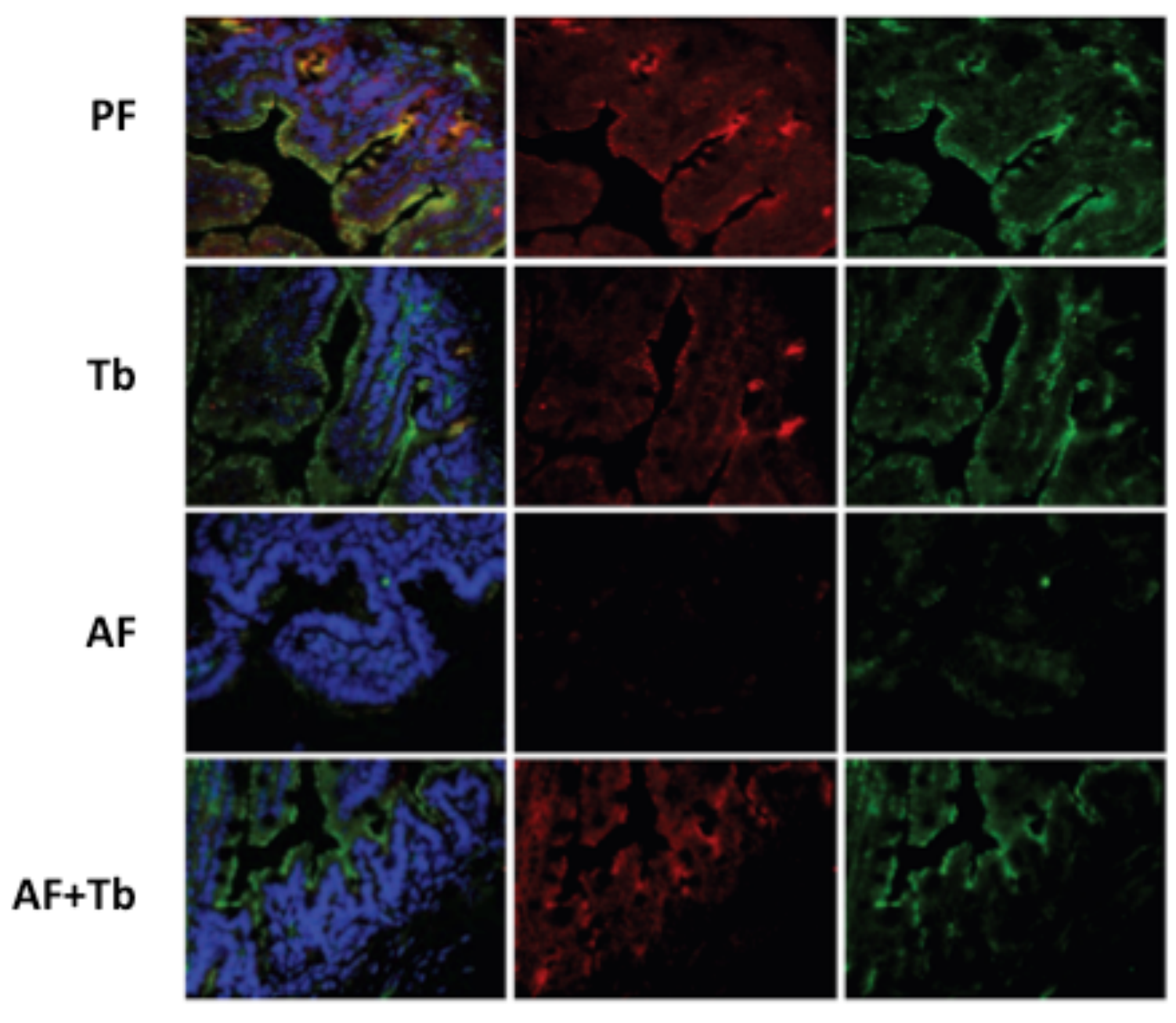

\section{Blue: Dapi; Red: ZO-1; Green: Occludin}

Fig.(2): Effect of tributyrin administration on intestinal tight junction protein expression: Mice were pair-fed with either control diet or ethanol $(5 \% \mathrm{v} / \mathrm{v})$ containing diet along with tributyrin $(2 \mathrm{~g} / \mathrm{kg}$ ) orally gavaged. Occludin (green) and ZO-1 (red) were visualized by immunohistochemistry in sections of colon frozen in OCT. Nuclei were counterstained with DAPI (blue). All images were acquired using a 409 objective. 


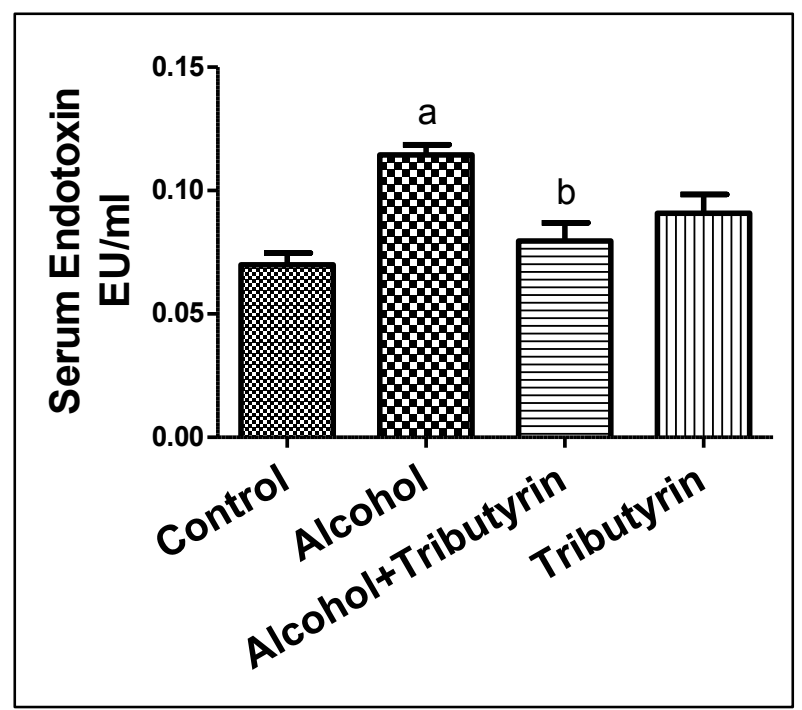

Fig. (3): Effect of tributyin administration on alcohol-induced endotoxemia: Mice were pair-fed with either control diet (PF) or ethanol (AF) $(5 \% \mathrm{v} / \mathrm{v})$ containing diet along with tributyrin $(\mathrm{Tb})(2 \mathrm{~g} / \mathrm{kg})$ orally gavaged. Serum endotoxin levels assessed by biochemical assay were significantly elevated in alcohol treated mice and attenuated by tributyrin administration. Data are expressed as mean \pm SEM, $n=4$ to 6 animals/per group. $a=$ compared to control, $b=$ compared to alcohol. 


\section{Tributyrin attenuates alcohol-induced hepatic steatosis}

Oral administration of tributyrin has been shown to increase concentration of butyrate in the portal vein and prevent lipopolysaccharide-induced liver injury in rats (Miyoshi et al). Since our earlier data showed that tributyrin decreases endotoxemia in alcohol fed mice, we examined its effects on hepatic steatosis which is a primary pathological consequence of chronic ethanol consumption. Histological analysis of the livers as shown by H\&E (Fig. 4) and Oil red O (Fig.5) staining revealed increase steatosis in livers of chronic alcohol fed mice. Interestingly, alcohol intake-induced fat accumulation was significantly attenuated by the tributyrin treatment. Effect of tributyrin on hepatic steatosis was further confirmed by hepatic triglyceride (Fig.6) and Non-esterified free fatty acids (NEFA) analysis (Fig.6). As expected, hepatic triglycerides and free fatty acids were increased in alcohol-fed mice compared to pair fed animals However, in correspondence with the immunohistochemistry of the livers, tributyrin treatment significantly blunted alcohol-induced hepatic triglycerides as well as free fatty acids. These results provides compelling evidence that oral administration of tributyrin can prevent development of alcoholic fatty liver in mice. 


\section{Control}
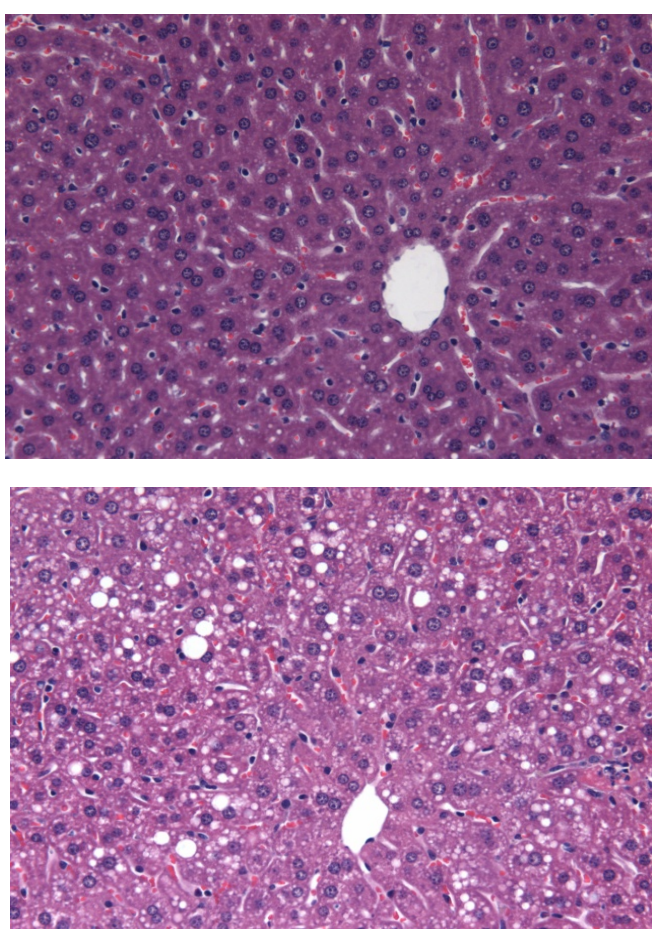

Alcohol

\section{Tributvrin}
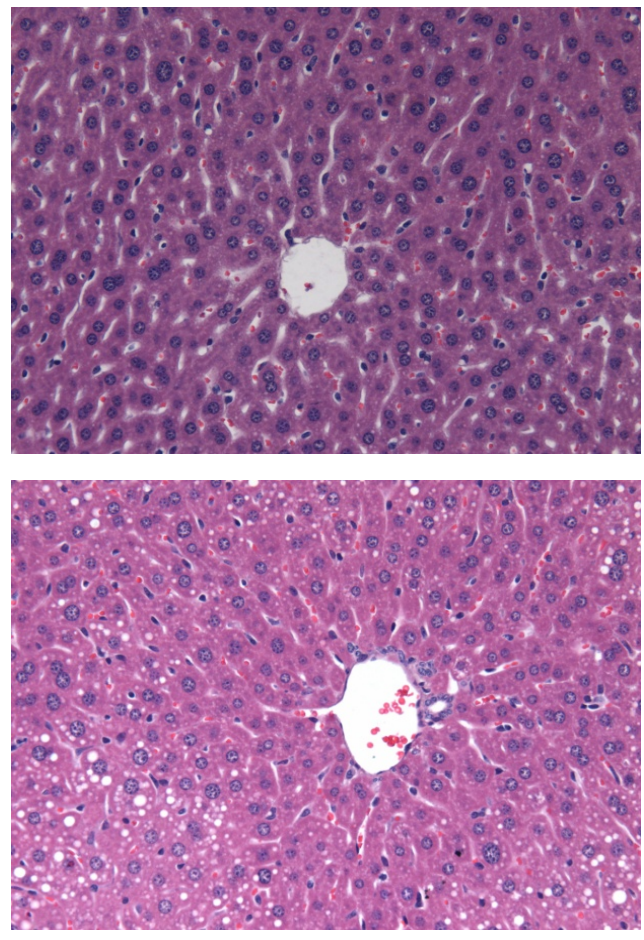

Alcohol+Tributvrin

Fig.(4): Effect of Tributyrin administration on alcohol-induced fatty liver. Mice were pair-fed with either control diet or ethanol $(5 \% \mathrm{v} / \mathrm{v})$ containing diet along with tributyrin $(2 \mathrm{~g} / \mathrm{kg})$ orally gavaged. Representative micrographs are shown depicting H\&E staining (magnification 20X) of liver sections revealing fat accumulation in the liver of alcohol fed mice 
Control
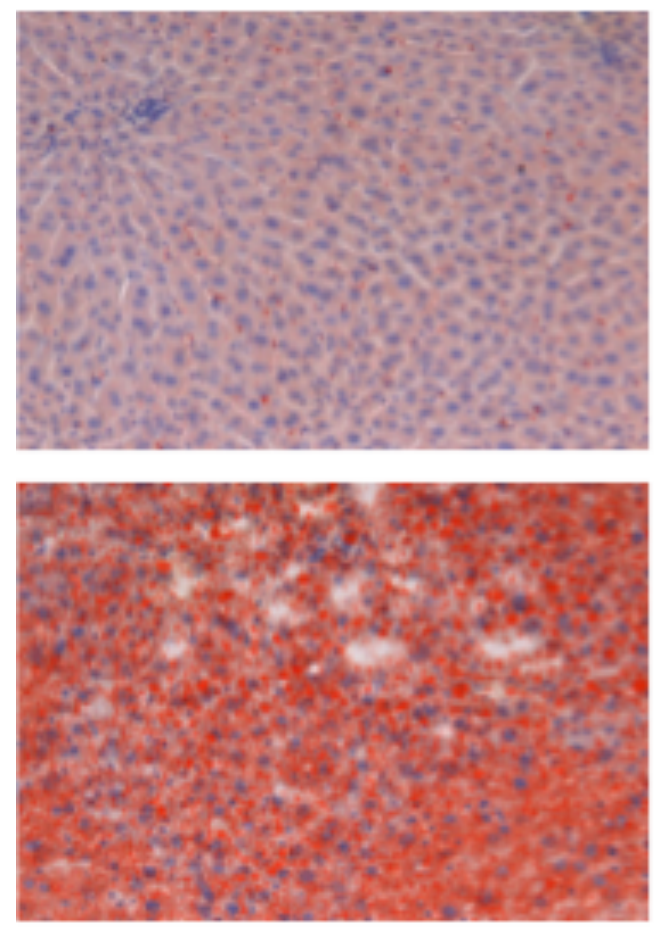

Alcohol
Tributyrin
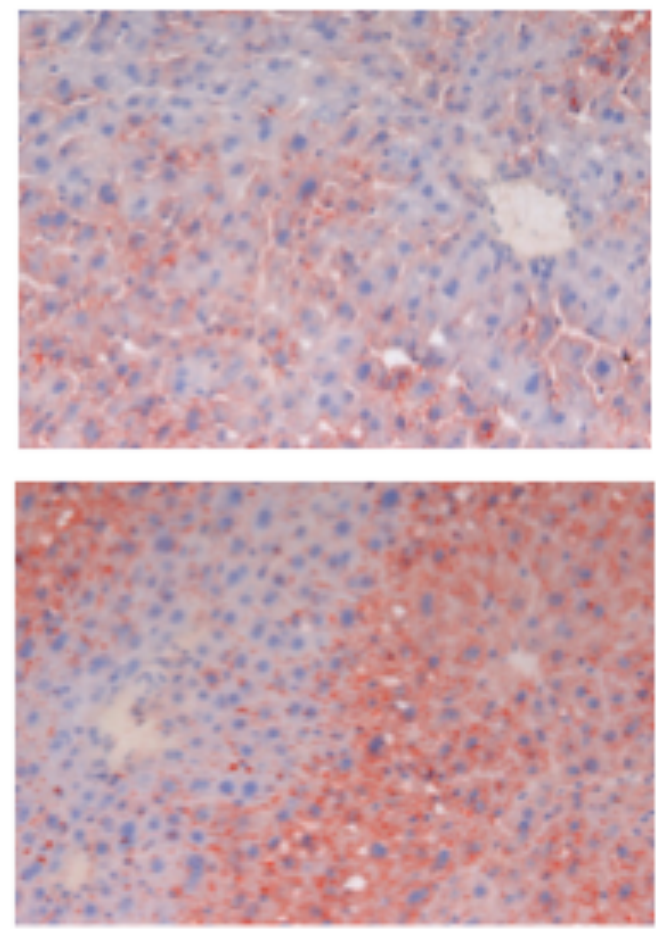

Alcohol+Tributyrin

Fig.(5): Effect of Tributyrin administration on alcohol-induced fatty liver. Mice were pair-fed with either control diet or ethanol $(5 \% \mathrm{v} / \mathrm{v})$ containing diet along with tributyrin $(2 \mathrm{~g} / \mathrm{kg})$ orally gavaged. Representative micrographs are shown depicting Oil-Red $\mathrm{O}$ staining (magnification 20X) of liver sections revealing fat accumulation in the liver of alcohol fed mice 

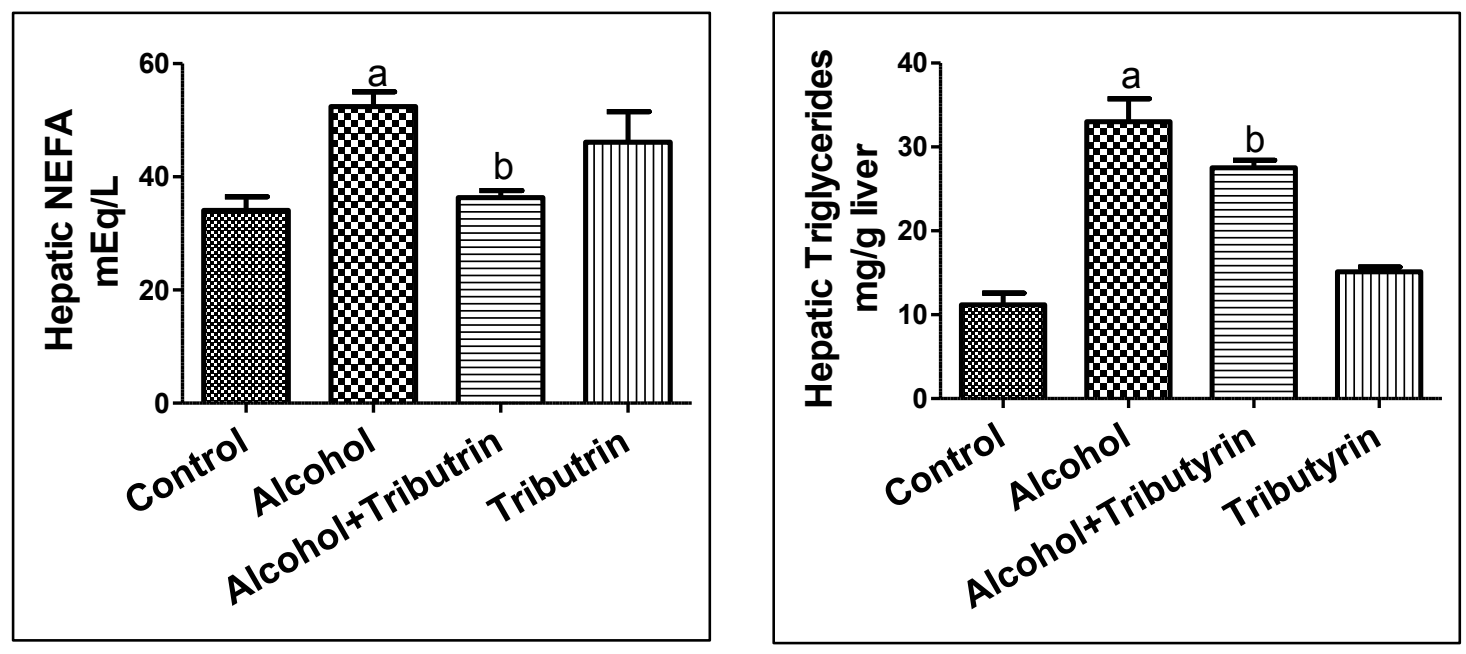

Fig.(6): Effect of tributyrin administration on alcohol-induced hepatic steatosis as assessed by hepatic free fatty acids and triglyceride : Mice were pair-fed with either control diet (PF) or ethanol (AF) $(5 \% \mathrm{v} / \mathrm{v})$ containing diet along with tributyrin $(\mathrm{Tb})(2 \mathrm{~g} / \mathrm{kg})$ orally gavaged. Data are expressed as mean $\pm \mathrm{SEM}, \mathrm{n}=$ 4 to 6 animals/per group. $a=$ compared to control, $b=$ compared to alcohol. 
Tributyrin administration prevents the decrease of CPT-1 gene expression in alcohol-fed mice

We and others have earlier demonstrated that alcohol-mediated down-regulation of CPT-1 plays a major role in the development of micro as well as macrovesicular steatosis $[17-19,144,145]$. Biochemically, CPT-1 is a rate-limiting enzyme in mitochondrial free fatty acid $\beta$-oxidation. Hence, to explore the potential mechanism through which tributyrin attenuates alcohol-induced hepatic steatosis, we examined CPT-1 mRNA levels by real time PCR from animal livers. Similar to our binge ethanol model, chronic ethanol feeding also significantly suppressed hepatic CPT-1 mRNA expression (Fig. 7) and led to down regulation in its protein expression as shown by the anti-CPT-1 staining in liver sections (Fig.8). Interestingly, tributyrin administration alone showed the highest expression at the mRNA level as compared to pair-fed control and alcohol fed animals. These data suggest that tributyrin administration increases $\beta$-oxidation by increasing hepatic CPT-1 gene expression and thus mitigates alcohol-induced hepatic steatosis. 


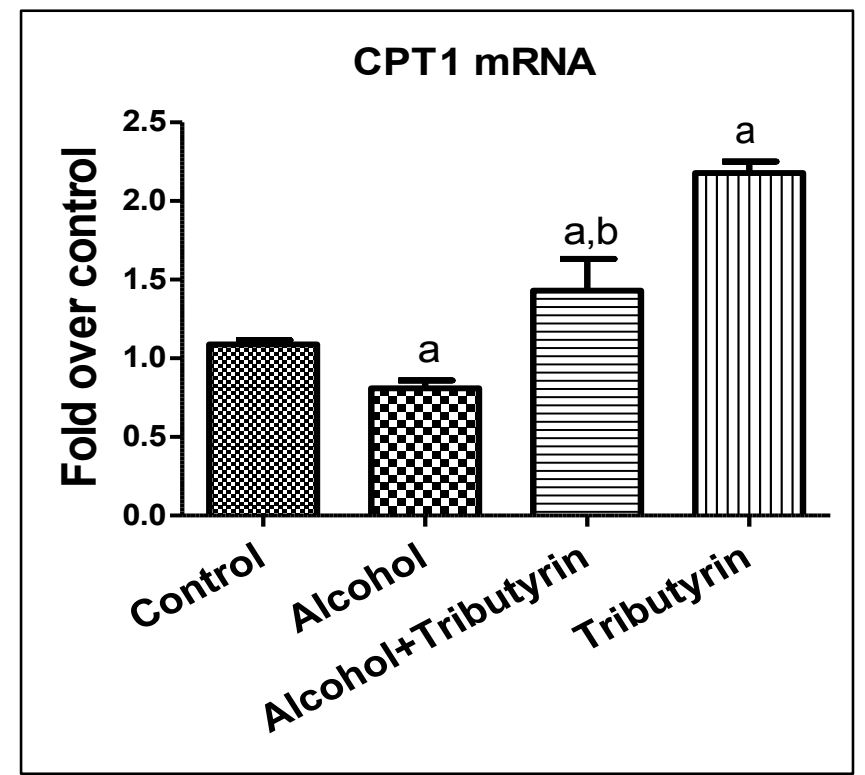

Fig.(7): Effect of tributyrin administration on alcohol induced down-regulation of CPT-1 gene expression: CPT-1 mRNA levels were significantly downregulated in alcohol fed mice and was further restored and increased by tributyrin treatment. CPT-1 mRNA levels were analyzed by RT-qPCR and normalized to $\beta$-actin mRNA. Data are expressed as mean \pm SEM, $n=5$ animals/per group. $a=$ compared to control, $b=$ compared to alcohol. 


\section{Pair-Fed}
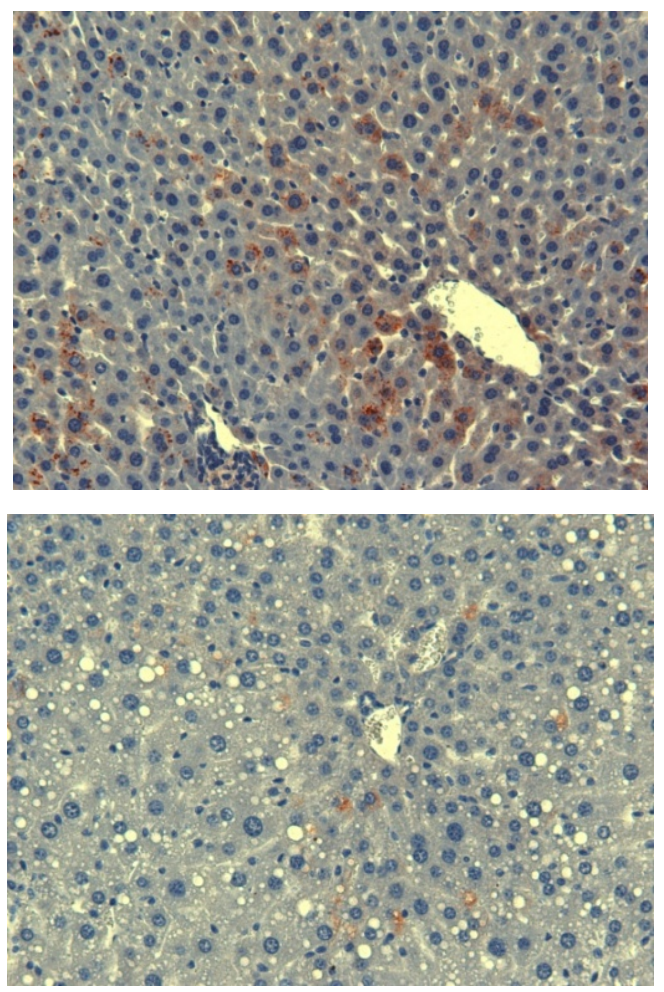

Alcohol
Tributyrin
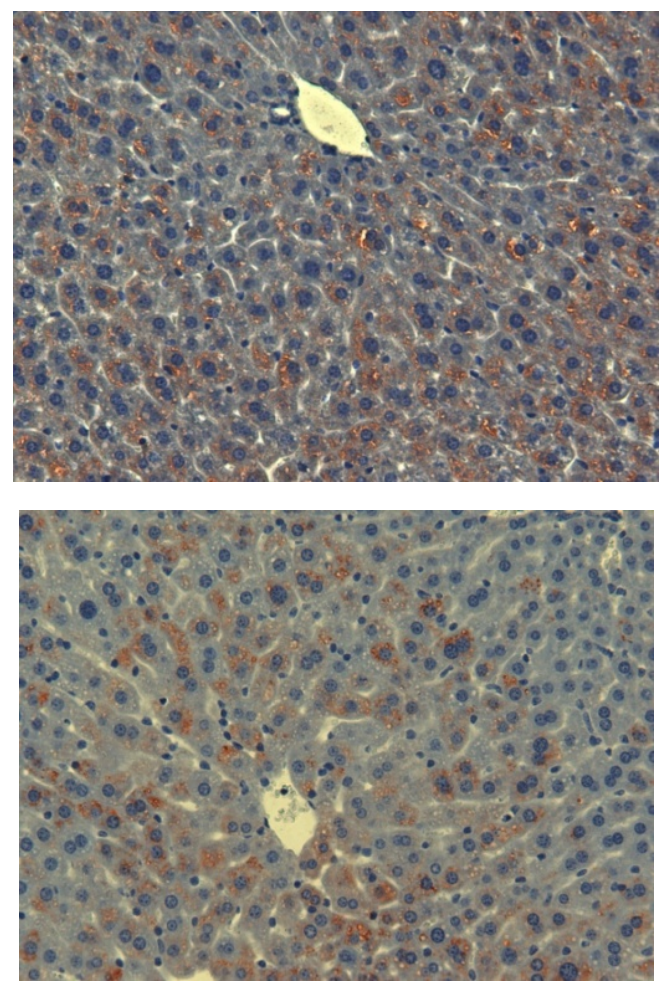

Alcohol+Tributyrin

Fig.(8): Effect of Tributyrin administration on hepatic CPT-1 protein expression in mice liver. Mice were pair-fed with either control diet or ethanol $(5 \% \mathrm{v} / \mathrm{v})$ containing diet along with tributyrin $(2 \mathrm{~g} / \mathrm{kg})$ orally gavaged. Immunohistochemical staining with anti-CPT-1 antibody (magnification 20X) demonstrated that hepatic CPT-1 protein levels (staining) were decreased in the liver of alcohol-fed mice and restored by tributyrin administration. 


\section{Evaluation of Alcohol and Butyrate Mediated Transcriptional Regulation of CPT-1 Gene Expression}

To determine the mechanisms underlying the chronic alcohol consumption mediated suppression of CPT-1 gene expression and the therapeutic effects of butyrate, we performed in vitro analysis using freshly isolated primary hepatocytes. As mentioned earlier orally administered tributyrin has shown to increase butyrate levels in the hepato-portal circulation. Hence butyrate was used in all the in vitro studies. Primary hepatocytes were either left untreated (UT) or treated with $50 \mathrm{mM}$ ethanol (for $48 \mathrm{hrs}$ ) with or without sodium butyrate (NaB; $2 \mathrm{mM}) . \mathrm{NaB}$ was added 30 min prior to ethanol treatment. The data obtained from the primary hepatocytes confirmed our in vivo findings on CPT-1 gene expression. Indeed, NaB pretreatment not only prevented the alcoholinduced decrease but further enhanced CPT-1 mRNA expression (Fig. 9).

\section{Effect of Alcohol and Butyrate on PGC-1 - a key co-activator of CPT-1 gene expression}

PGC-1 is an essential transcriptional co-activator of hepatic CPT-1 gene expression and is known to be downregulated by alcohol [34, 42, 49]. 48 hours of alcohol significantly suppressed PGC-1 gene expression, whereas butyrate pretreatment not only reversed but also robustly induced PGC-1 gene expression in primary hepatocytes. (Fig.10).

Biological effects of butyrate are mediated by its ability to primarily function as a Class I/II HDAC inhibitor which can increase histone acetylation and activate gene expression [146]. Hence, to address the butyrate effect of enhancing PGC-1 mRNA expression, promoter histone acetylation was 
assessed. Specifically, histone acetylation in the promoter region encompassing the cAMP response element (CRE) that binds CREB, a known transcription factor for PGC-1 expression was examined (Fig.11). Chromatin was prepared from the rat primary hepatocytes treated with alcohol $50 \mathrm{mM}$ and with/without pretreatment with $\mathrm{NaB} 2 \mathrm{mM}$ as well as from $\mathrm{NaB}$ alone for 12 hours. ChIP analysis was performed using an $\mathrm{Ab}$ that selectively recognizes $\mathrm{H} 3 \mathrm{~K} 9 \mathrm{Ac}$. Commensurate with its effect on PGC-1 mRNA, butyrate was observed to prevent alcohol induced decrease in promoter H3K9Ac levels and in fact increased the levels over base line (Fig.12). 


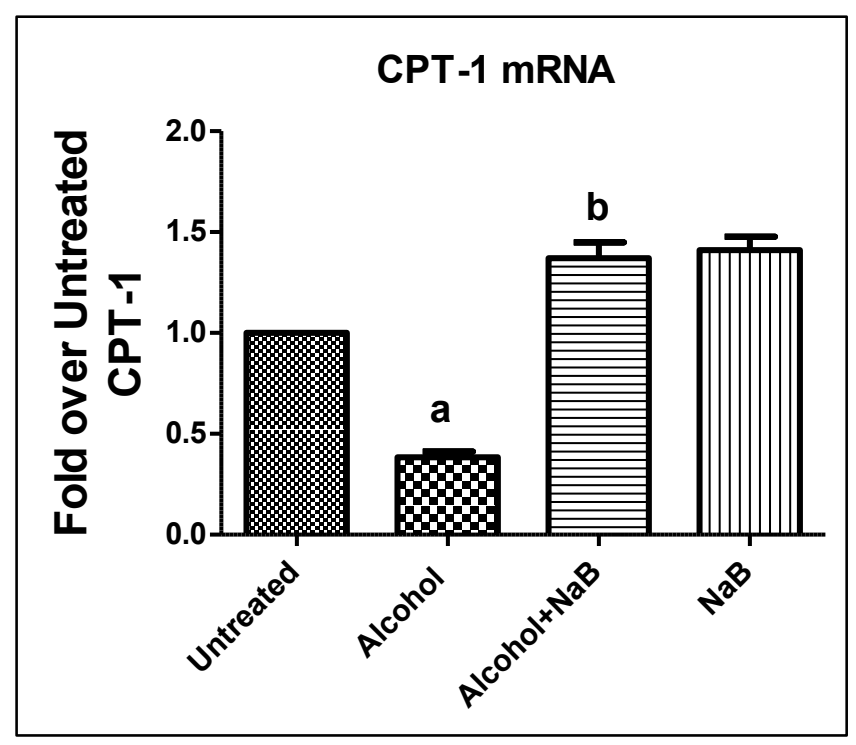

Fig.(9): Effect of butyrate on alcohol mediated down-regulation of CPT-1 gene expression. Primary hepatocytes were untreated (UT) or treated with $50 \mathrm{mM}$ alcohol (A) for $48 \mathrm{~h}$. Cells were pretreated with sodium butyrate for 30 min prior to alcohol. CPT-1 mRNA levels were analyzed by RT-qPCR and normalized to $\beta$-actin mRNA. Data are expressed as mean \pm SEM, $n=3$. $a=$ compared to untreated, $b=$ compared to alcohol. 


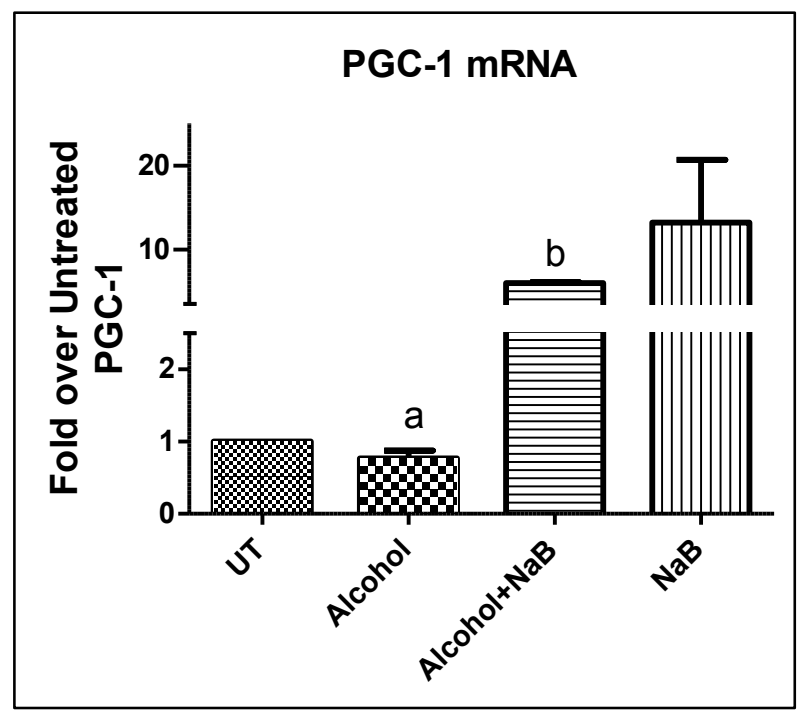

Fig.(10): Effect of butyrate on alcohol mediated down-regulation of PGC-1 gene expression Primary hepatocytes were untreated (UT) or treated with $50 \mathrm{mM}$ alcohol $(A)$ for $48 \mathrm{~h}$. Cells were pretreated with sodium butyrate for $30 \mathrm{~min}$. PGC-1 mRNA levels were analyzed by RT-qPCR and normalized to $\beta$-actin mRNA. Data are expressed as mean \pm SEM, $n=3$. $a=$ compared to untreated, $b=$ compared to alcohol. 


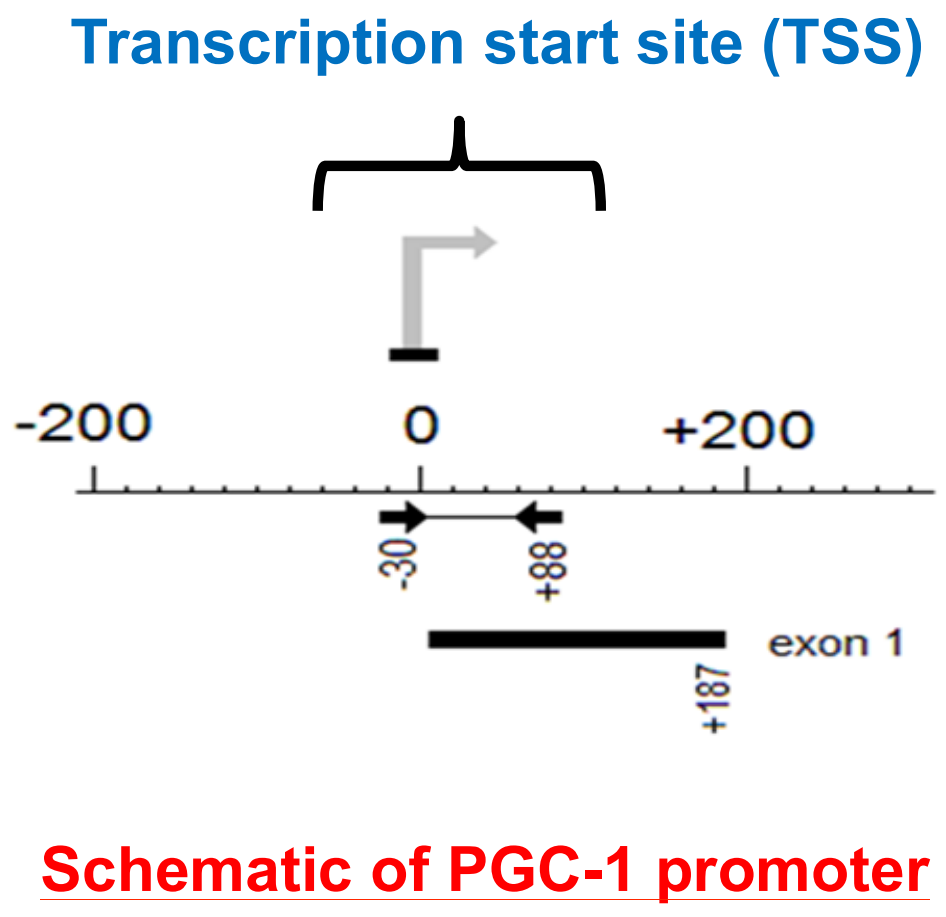

Fig.(11) PGC-1 promoter schematic. Location of ChIP PCR primer pair for analysis of epigenetic modifications is shown in the schematic with respect to the +1 start site REFSEQ NM_000639.1 


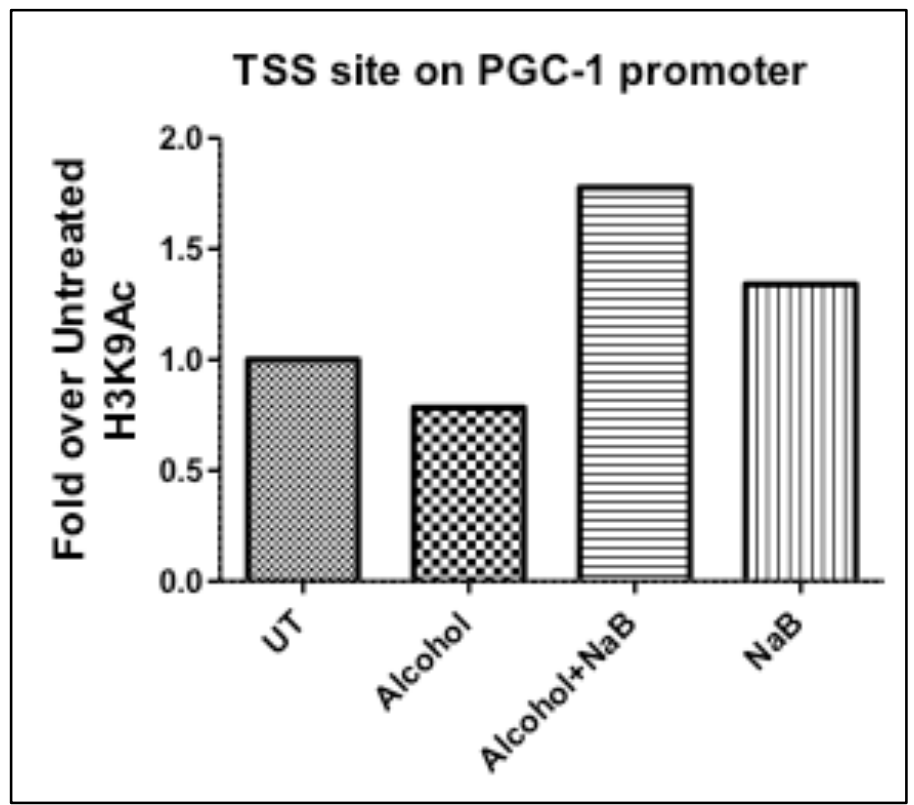

Fig.(12) H3K9Ac enhancement at PGC-1 promoter by butyrate: Primary hepatocytes were untreated (UT) or treated with $50 \mathrm{mM}$ alcohol for $12 \mathrm{~h}$. Sodium butyrate was pretreated for $30 \mathrm{~min}$. Chromatin fragments were immunoprecipitated with $\mathrm{H} 3 \mathrm{~K} 9 \mathrm{Ac}$ antibody and ChIP-qPCR was performed using primer pair specific for TSS site on PGC-1 promoter. A representative PCR data is shown. Non-immunoprecipitated chromatin was used as input. 


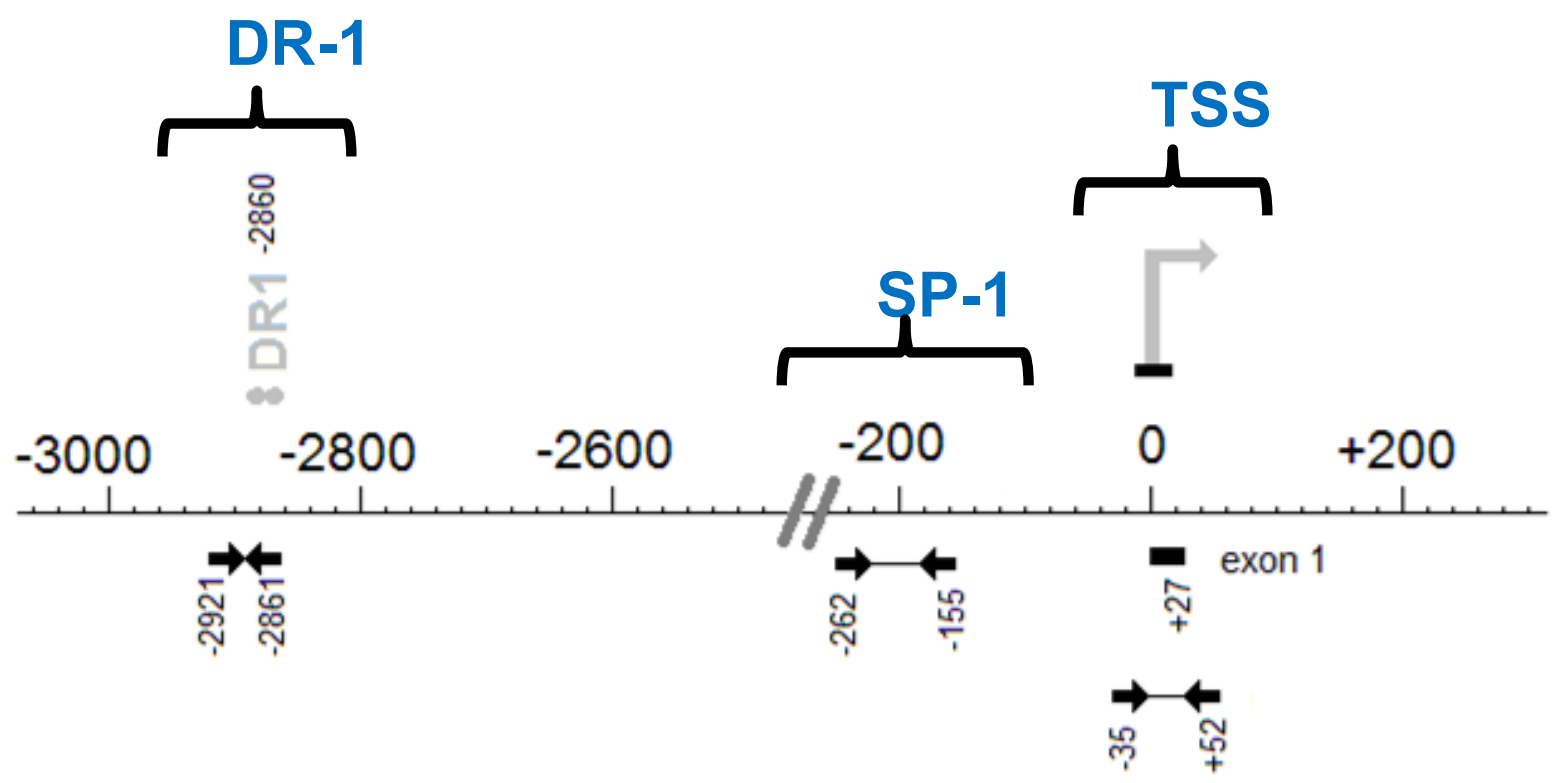

\section{Schematic of CPT-1 promoter}

Fig.(13) CPT-1 promoter schematic: Locations of key transcriptionally active regions and ChIP PCR primer pairs for analysis of epigenetic modifications are denoted. The coordinate locations shown are with respect to the +1 start site REFSEQ NM_000639.1 


\section{Effect of Alcohol and Butyrate on CPT-1 Transcriptional Mechanisms Effect on the recruitment of co-activators PGC-1and p300}

PGC-1 is a key transcriptional coactivator of CPT-1 gene expression. As a coactivator, PGC-1 can recruit and facilitate interactions with other coactivators such histone acetyl transferases (HATS; e.g. SRC-1, p300) that can induce chromatin remodeling via histone acetylation $[147,148]$. Hence, in correlation with the observed CPT-1 and PGC-1 mRNA expression we examined the effect of alcohol and butyrate on PGC-1 and p300 protein recruitment to the transcriptionally relevant CPT-1 promoter regions denoted as DR-1 site and SP-1 site (Fig. 13) Chromatin from alcohol and $\mathrm{NaB}$ treated hepatocytes was obtained as mentioned above and ChIP analysis was performed using PGC-1 specific antibodies. Relative to CPT-1 mRNA expression, alcohol decreased PGC-1 binding which was prevented by $\mathrm{NaB}$ (Fig.14). Following PGC-1, recruitment of HAT - p300 to the relevant CPT-1 promoter region was evaluated. Proportional to PGC-1 recruitment and its coactivator function, alcohols diminishing effect on p300 binding was counteracted by $\mathrm{NaB}$ resulting in an increase in its binding (Fig.15).

Subsequent to examining the effects of alcohol and butyrate on PGC-1 and p300 recruitment, we examined their effect on promoter histone modifications as pertinent epigenetic mechanisms dictating CPT-1 transcription. Promoter histone modifications were examined by ChIP qPCR analysis [149] using PCR primer sets for region that overlaps the transcription state site (TSS site). Specifically, the status of histone $\mathrm{H} 3$ lysine 9 acetylation (H3K9Ac) was assessed which plays a key role in the transcriptional activation of gene expression. 
Chromatin from primary hepatocytes treated with ethanol and $\mathrm{NaB}$ was evaluated by ChIP analysis by using an Ab that selectively recognizes H3K9Ac. Commensurate with the observed decrease in CPT-1 mRNA levels as well as PGC-1 and p300 binding, ethanol treatment led to a decrease in the promoter H3K9Ac levels indicative of a transcriptionally repressive chromatin state. In comparison, $\mathrm{NaB}$ pre-treatment not only inhibited the ethanol induced decrease, but also increased H3K9Ac levels over base-line. Interestingly, NaB alone markedly increased CPT-1 promoter H3K9Ac levels and induced CPT-1 transcription (Fig.16). These data suggest that the protective effect of $\mathrm{NaB}$ is significantly mediated through its ability to function as an HDAC inhibitor. 


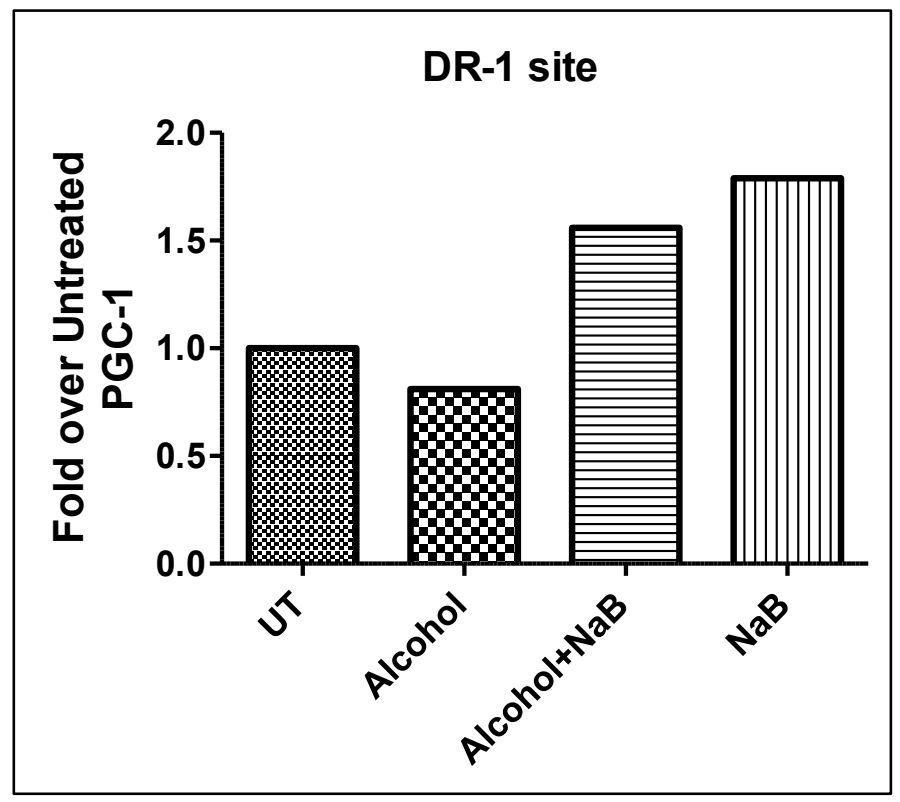

Fig: (14) Increase in PGC-1 binding at the CPT-1 promoter by butyrate: Primary hepatocytes were untreated (UT) or treated with $50 \mathrm{mM}$ alcohol for $12 \mathrm{~h}$. Sodium butyrate was pretreated for $30 \mathrm{~min}$ prior to alcohol. Chromatin fragments were immunoprecipitated with PGC-1 antibody and ChIP-qPCR was performed using primer pair specific for DR-1 site on CPT-1 promoter. Representative PCR data is shown. Non-immunoprecipitated chromatin was used as input to normalize the data. 


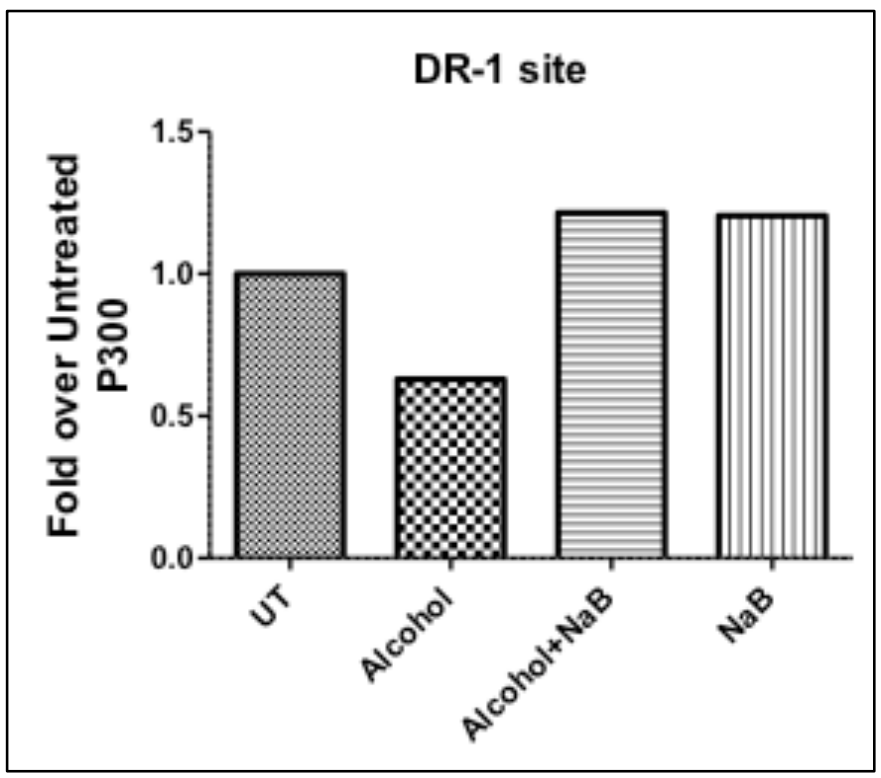

Fig.(15) Increase in p300 recriuitment by PGC-1 at the CPT-1 promoter by butyrate: Primary hepatocytes were untreated (UT) or treated with $50 \mathrm{mM}$ alcohol for $12 \mathrm{~h}$. Sodium butyrate was pretreated for $30 \mathrm{~min}$. Chromatin fragments were immunoprecipitated with p300 antibody and ChIP-qPCR was performed using primer pairs specific for DR-1 site on CPT-1 promoter. Representative PCR data is shown. Non-immunoprecipitated chromatin was used as input to normalize the data. 


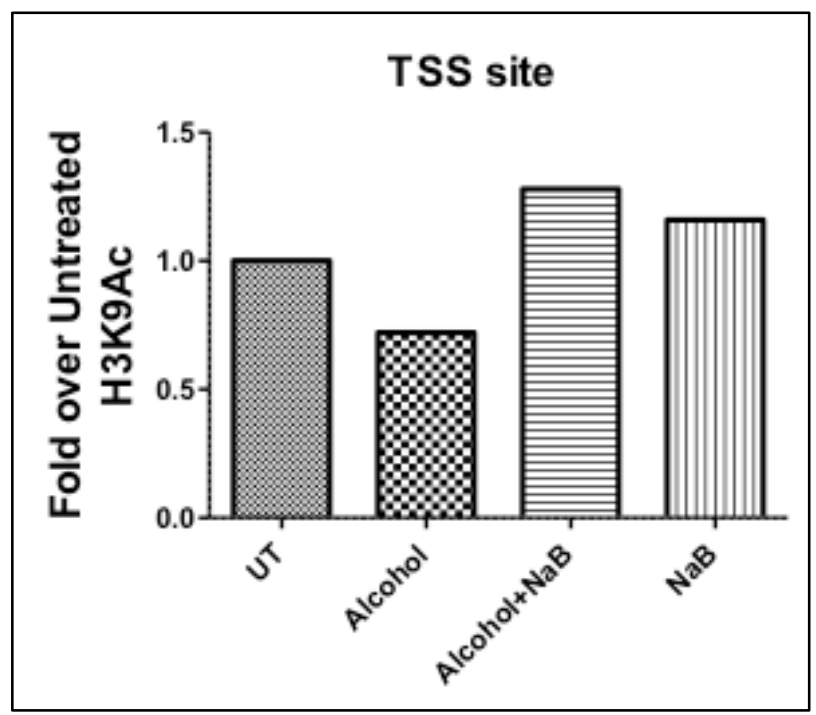

Fig.(16) Increase in H3K9Ac levels at the TSS site of the CPT-1 promoter by butyrate: Primary hepatocytes were untreated (UT) or treated with $50 \mathrm{mM}$ alcohol for $12 \mathrm{~h}$. Sodium butyrate was pretreated for $30 \mathrm{~min}$ prior to alcohol. Chromatin fragments were immunoprecipitated with H3K9Ac antibody and ChIP-qPCR was performed using primer pairs specific for TSS site and SP-1 site on CPT-1 promoter. A representative PCR data is shown. Nonimmunoprecipitated chromatin was used as input. 


\section{Effect on CPT-1 transcription pre-initiation complex}

Earlier studies have demonstrated that CPT-1 is a TATA-less gene and transcription factor $\mathrm{Sp} 1$ is significantly involved in the regulation of basal as well as inducible transcriptional activation of CPT-1 gene expression [43, 44]. Hence, having determined the impact of alcohol and butyrate on the recruitment of coactivators and promoter histone acetylation affecting CPT-1 mRNA expression, we then examined the effects on Sp1 and RNA Pol II binding that constitutes the relevant components of the CPT-1 transcription pre-initiation complex. Chromatin from primary hepatocytes treated with ethanol and $\mathrm{NaB}$ was evaluated by ChIP analysis by using antibodies that selectively recognize Sp1 and RNA Pol II. Sp1 binding was examined at Sp1 site and RNA Polll binding was examined at the TSS site (Fig.13). In correlation with the decrease in CPT-1 mRNA expression, alcohol induced a disruption of the transcriptional preinitiation complex as indicated by the decline in transcription factor - Sp1 binding and recruitment of RNA Pol II at the CPT-1 promoter (Fig.17, 19). Importantly, butyrate in correlation with an increase in CPT-1 promoter histone acetylation indicative of an increase in the transcriptionally active state, led to an increase in Sp1 binding and RNA Pol II recruitment and recommencement of the formation of the transcriptional pre-initiation complex. Moreover Sp1 is known to promote transcription by interacting and recruiting p300 [150]. Indeed, a butyrate mediated increase in $\mathrm{p} 300$ binding to the Sp1 region of the CPT-1 promoter was also observed in alcohol treated hepatocytes (Fig.18). 
These data strongly suggest that p300 recruitment via Sp1, besides PGC-1, could have also contributed to the net dynamic increase in CPT-1 promoter histone acetylation and transcriptional activation.

Overall, oral administration of tributyrin significantly protects gut barrier function and attenuates chronic alcohol-induced hepatic steatosis. Specifically, tributyrin induces transcriptionally permissive chromatin modifications at the CPT-1 promoter and thus improves CPT-1 gene expression and $\beta$-oxidation in the chronic alcohol fed animals. 


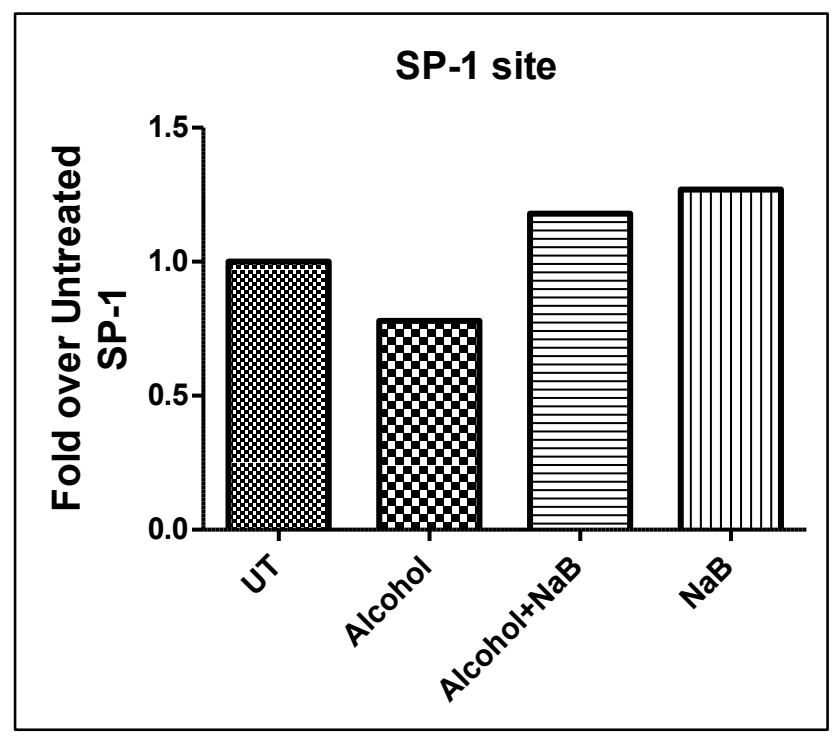

Fig.(17) Increase in Sp-1 binding at the Sp-1 site on the CPT-1 promoter by butyrate: Increase in PGC-1 binding at the CPT-1 promoter by butyrate Primary hepatocytes were untreated (UT) or treated with $50 \mathrm{mM}$ alcohol for $12 \mathrm{~h}$. Sodium butyrate was pretreated for $30 \mathrm{~min}$. Chromatin fragments were immunoprecipitated with SP-1 antibody and ChIP-qPCR was performed using primer pair specific for SP-1 site on CPT-1 promoter. Representative PCR data is shown. Non-immunoprecipitated chromatin was used as input to normalize the data. 


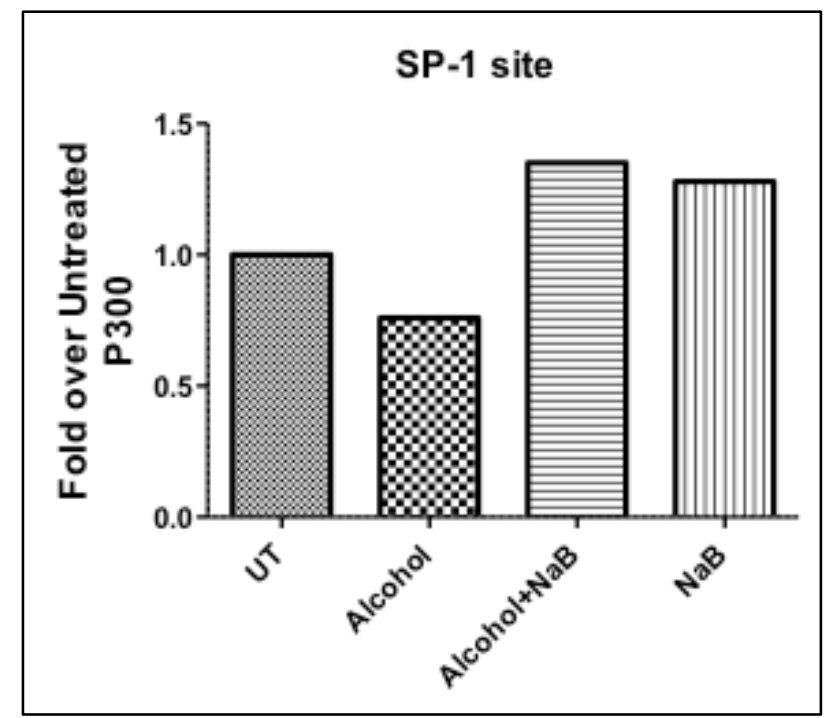

Fig.(18) Increase in p300 recruitment by SP-1 at the SP-1 site on the CPT-1 promoter: Primary hepatocytes were untreated (UT) or treated with $50 \mathrm{mM}$ alcohol for $12 \mathrm{~h}$. Sodium butyrate was pretreated for $30 \mathrm{~min}$. Chromatin fragments were immunoprecipitated with p300 antibody and ChIP-qPCR was performed using primer pairs specific for SP-1 site on CPT-1 promoter. Representative PCR data is shown. Non-immunoprecipitated chromatin was used as input to normalize the data. 


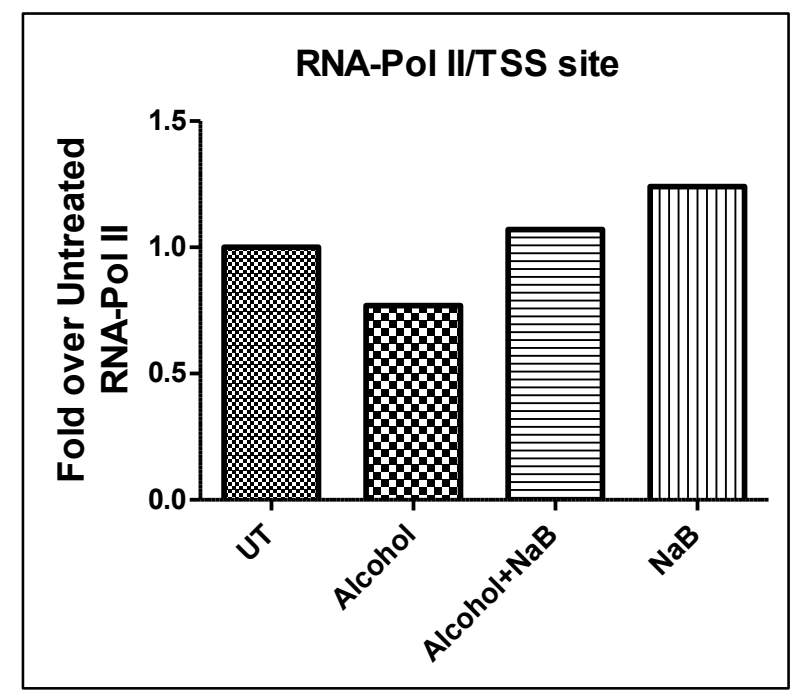

Fig.(19) Increase in RNA pol II at the TSS site on the CPT-1 promoter: Primary hepatocytes were untreated (UT) or treated with $50 \mathrm{mM}$ alcohol (A) for $12 \mathrm{~h}$.

Sodium butyrate was pretreated for $30 \mathrm{~min}$. Chromatin fragments were immunoprecipitated with RNA-Pol II antibody and ChIP-qPCR was performed using primer pair specific for TSS site on CPT-1 promoter. Representative PCR data is shown. Non-immunoprecipitated chromatin was used as input to normalize the data. 


\section{Tributyrin administration protects from alcohol-induced hepatic inflammation and injury}

Studies have shown that $10-30 \%$ heavy drinkers progresses from hepatic steatosis to hepatic inflammation and injury. Activation of innate immune response either by kupffer cells which are resident macrophages or infiltrating macrophages and resultant pro-inflammatory cytokine production such as TNF- $\alpha$, IL-8, IL-1 $\beta$ have been well documented in alcoholic liver disease. Since tributyrin showed protection against alcohol induced steatosis we next investigated its impact on hepatic inflammation and injury.

\section{Tributyrin inhibits the alcohol-mediated macrophage activation and pro- inflammatory cytokine -TNF- $\alpha$ expression}

The effect of tributyrin on macrophage activation was evaluated by immunohistochemistry for F4/80-positive macrophages in the liver tissue (Fig.20). Data showed that there are more F4/80 positive macrophages as seen by brown stained cells in alcohol fed animals but that number was markedly less in the tributyrin and alcohol together treated mice. Additionally, the macrophage activation was further confirmed by measuring pro-inflammatory cytokine, tumor necrosis factor- $\alpha$ (TNF- $\alpha$ ) production (Fig.21). In accordance with macrophage activation, the TNF- $\alpha$ mRNA levels were high at 2 weeks alcohol feeding but were significantly decreased in mice that received tributyrin administration along with alcohol feeding. Tributyrin alone did show marginal increase in TNF- $\alpha$ mRNA levels but difference was not significant as compared to pair-fed controls. 


\section{Butyrate down-regulated LPS inducible TNF-a gene expression in RAW cells}

Since increase TNF- $\alpha$ expression was seen in two week alcohol fed animals and Aldred et al. have shown that after chronic alcohol consumption, macrophages/Kupffer cells demonstrate enhanced LPS-stimulated TNF- $\alpha$ production[151]. We next examine the effect of butyrate on LPS inducible TNF- $\alpha$ gene expression in RAW cells (Fig.22). The data showed that LPS (100ng/ml) treatment caused robust upregulation ( $\sim 20$ fold $)$ in the TNF- $\alpha$ mRNA levels as compared to untreated cells; however the Na-butyrate $(1 \mathrm{mM})$ pretreatment inhibited LPS-stimulated TNF- $\alpha$ gene expression. These data further support the mechanistic role of tributyrin in the down-regulation of alcohol induced hepatic inflammation. 


\section{Control}
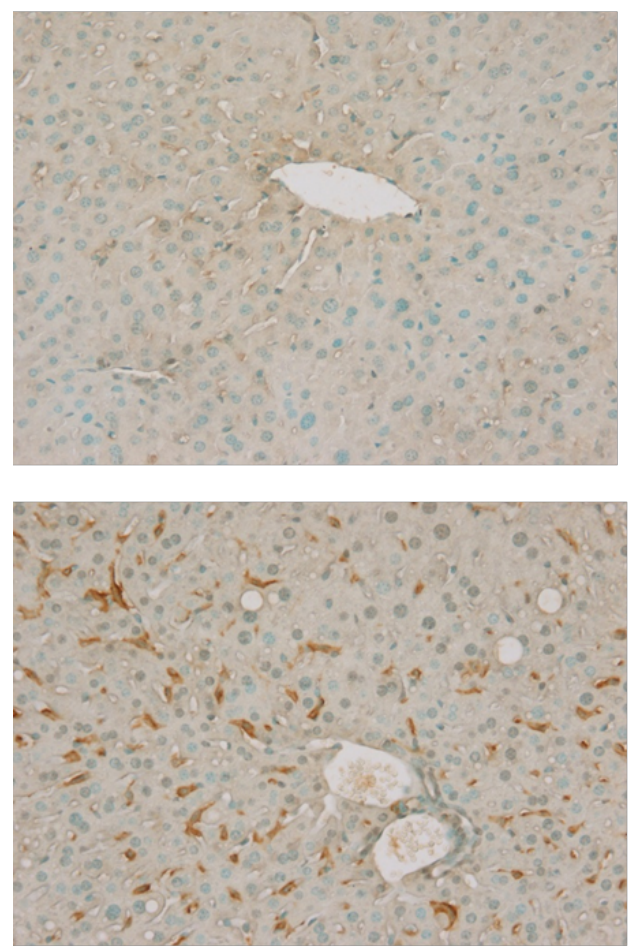

Alcohol
Tributyrin
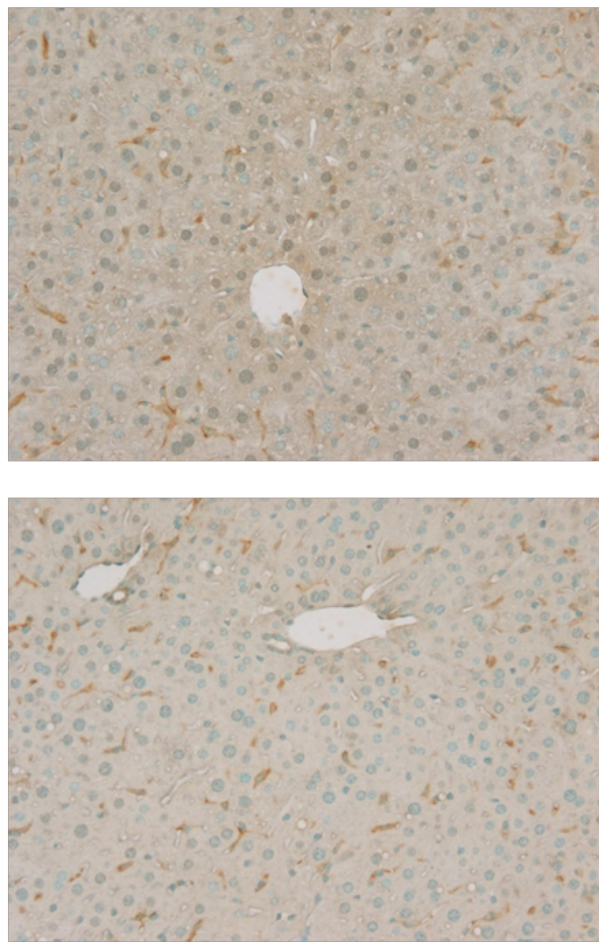

Alcohol+Tributyrin

Fig.(20) Effect of tributyrin administration on $\mathrm{F} 4 / 80$ positive cells in mice chronically fed alcohol for 2 weeks. Representative micrographs are shown depicting F4/80 staining of liver sections revealed increased macropage infiltration in liver of alcohol fed mice which is attenuated in tributyrin treated mice 


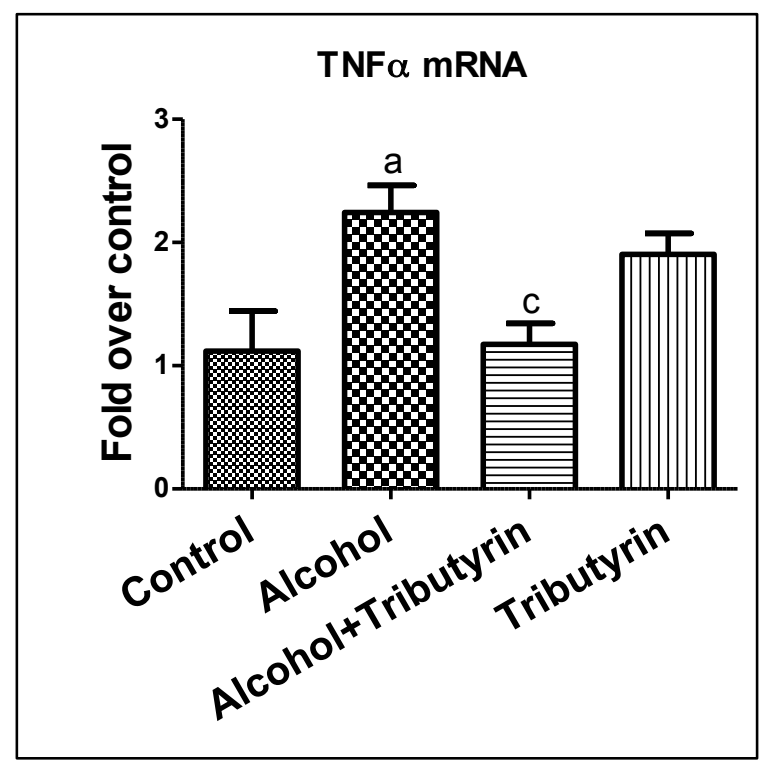

Fig.(21) Chronic alcohol-induced TNF- $\alpha$ gene expression: Alcohol significantly induced TNF- $\alpha$ gene expression which was attenuated by tributyrin treatment. TNF- $\alpha$ mRNA levels were analyzed by RT-qPCR and normalized to $\beta$-actin $m R N A$. Data are expressed as mean $\pm S E M, n=5$ animals/per group. $\mathrm{a}=$ compared to control and $\mathrm{b}=$ alcohol. 


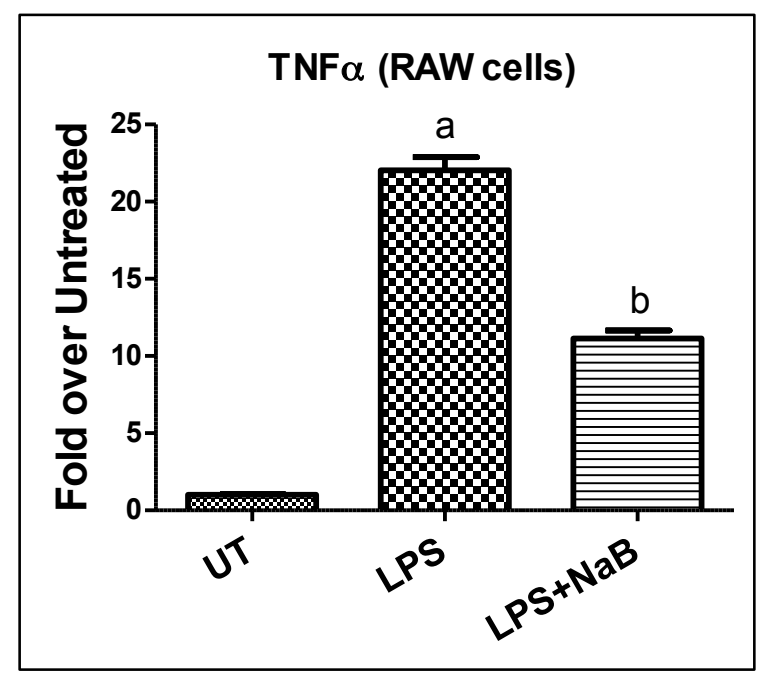

Fig.(22) LPS inducible TNF- $\alpha$ gene expression in RAW 264.7: LPS (100 $\mathrm{ng} / \mathrm{ml})$ inducible TNF- $\alpha$ gene expression was attenuated by butyrate $2 \mathrm{mM}$ tributyrin treatment. TNF- $\alpha$ mRNA levels were analyzed by RT-qPCR and normalized to $\beta$-actin mRNA. Data are expressed as mean \pm SEM, $n=3 . a=U T$ and $b=$ LPS 


\section{Tributyrin administration significantly attenuates chronic alcohol-induced}

\section{MCP-1 (CCL-2) gene expression in mice and rat hepatocytes}

MCP-1 upregulation plays a critical pathogenic role in the progression of alcoholinduced hepatic inflammation and injury in C57BL/6 mice as well as alcoholic hepatitis patients [93]. Hence in our animal model we investigated the effect of tributyrin/butyrate on the alcohol-induced regulation CCL-2 gene expression. In this model C57BL/6 mice were fed the Leiber-Decarli diet or its isocaloric control (Pair-fed PF) to determine the expression of CCL-2 in the whole liver. Two weeks of chronic alcohol feeding significantly induced ( $\sim 3$ fold) CCL-2 gene expression in the liver. This CCL-2 induction was further upregulated ( 7 fold) at the end 7 weeks. Tributyrin co-administration markedly suppressed the induction of alcohol-induced hepatic CCL-2 at 2 as well as 7 weeks of alcohol feeding to baseline pair-fed level (Fig.23). To isolate the role of hepatocytes in the alcoholinduced CCL-2 expression, rat primary hepatocytes were treated with alcohol (50mM) for $48 \mathrm{hrs}$ and TNF- $\alpha(20 \mathrm{ng} / \mathrm{ml})$ for $4 \mathrm{hrs}$. with/without pretreatment of butyrate (2mM, 30min). TNF- $\alpha$ exposure alone induced $\sim 10$ fold up-regulation in CCL-2 gene expression over untreated. Moreover, alcohol further primed the cells to TNF- $\alpha$ induced CCL-2 expression upto 35 fold as compared to untreated cells. Pre-treatment with $\mathrm{NaB}$ significantly attenuated $\mathrm{CCL}-2$ gene expression induced in response to TNF-a as well as combination of alcohol and TNF- $\alpha$ treatment (Fig. 24). These data indicates that tributyrin administration significantly down-regulates chronic-alcohol induced CCL-2 gene expression in 
the whole liver as well as in CCL-2 producing hepatocytes leading to decrease in alcohol-mediated hepatic inflammation and injury. 


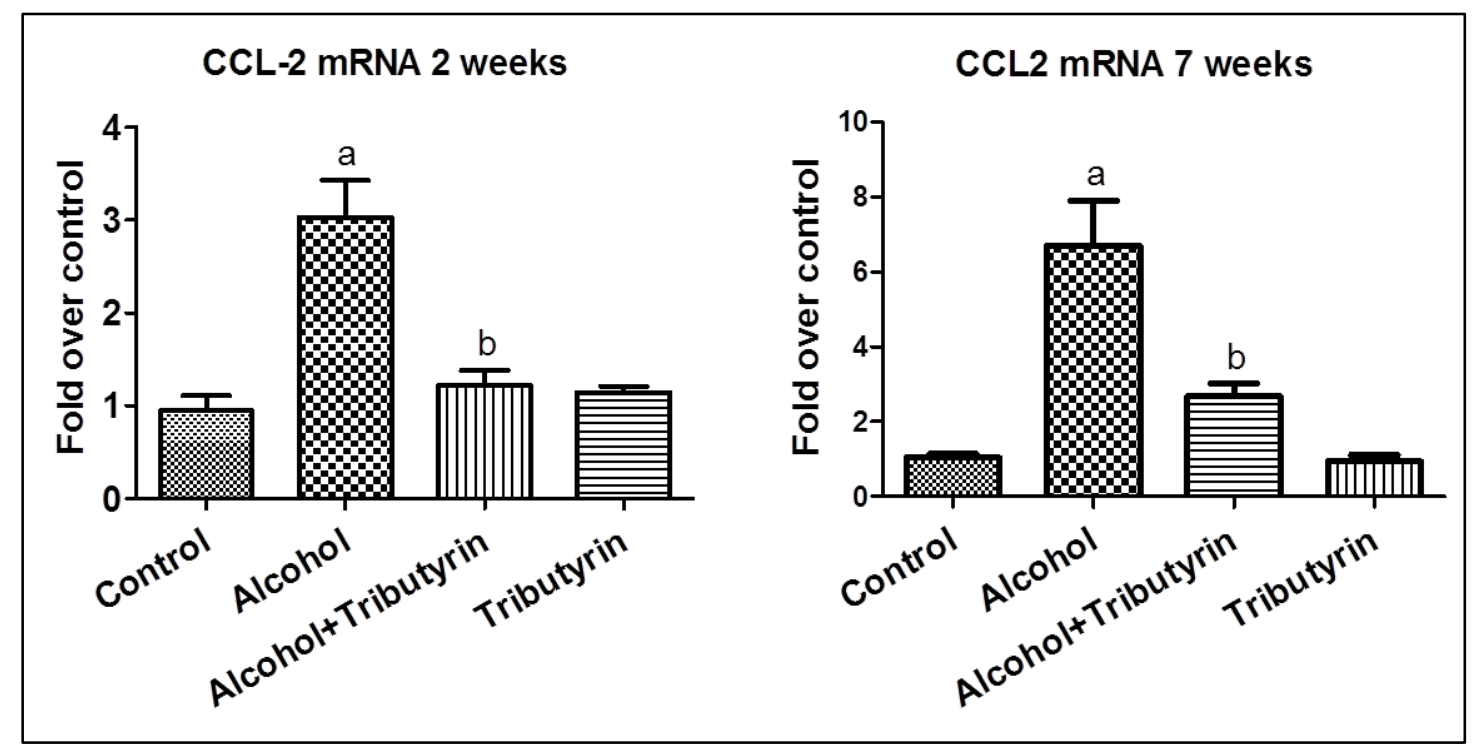

Fig.(23)Chronic alcohol induced CCL2 gene expression in alcohol fed mice: CCL2 mRNA levels were up-regulated in alcohol fed mice livers and was attenuated by tributyrin treatment. CCL2 mRNA levels were analyzed by RTqPCR and normalized to $\beta$-actin mRNA. Data are expressed as mean \pm SEM, $\mathrm{n}=5$ animals/per group. $\mathrm{p}$ value $\mathrm{a}=$ when compared to control and $\mathrm{b}=$ when compared to alcohol. 


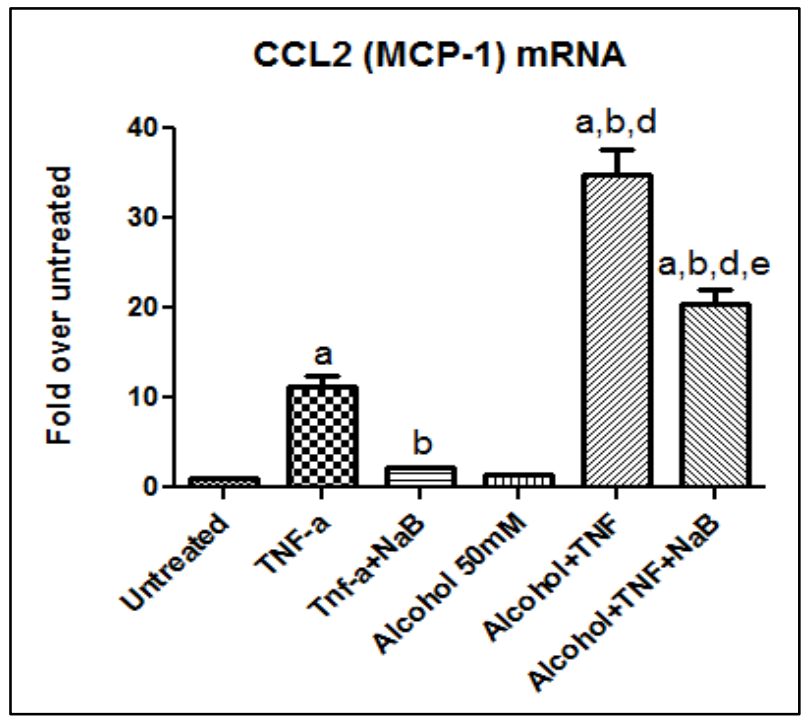

Fig.(24) Chronic alcohol induced CCL2 gene expression in rat primary hepatocytes: Hepatocytes were treated with alcohol $50 \mathrm{mM}$ for $48 \mathrm{hrs}$ and TNF$\alpha(20 \mathrm{ng} / \mathrm{ml})$ for $4 \mathrm{hrs}$ with/without Sodium butyrate (NaB). CCL2 mRNA levels were analyzed by RT-qPCR and normalized to $\beta$-actin mRNA. Data are expressed as mean \pm SEM, $n=3$. $p$ value a when compared to control; $b$ when compared to TNF- $\alpha$; $d$ when compared to alcohol and e when compared to Alcohol +TNF- $\alpha$. 


\section{Tributyrin administration significantly attenuates chronic alcohol-induced}

CXCL-2 (MIP-2) gene expression in mice

One of the key pro-inflammatory cytokines involved in modulating the inflammatory response is the CXC chemokine interleukin-8 (IL-8), which functions as a critical chemoattractant and activator for neutrophils, basophils, and T cells. Sustained consumption of ethanol was shown to be associated with elevated serum as well as hepatic MIP-2 [87, 152]. Hence we examined the expression of CXCL2 in the alcohol-fed mice with/without tributyrin coadministration. Two weeks of alcohol-feeding did not induce CXCL2 gene expression in the liver although a trend was observed. However, sustained alcohol consumption upto 7 weeks significantly induced CXCL-2 gene expression. This alcohol-induced CXCL2 gene expression was significantly suppressed by tributyrin administration suggesting that tributyrin attenuated hepatic inflammation caused due to CXCL2 cytokine production (Fig.25). 


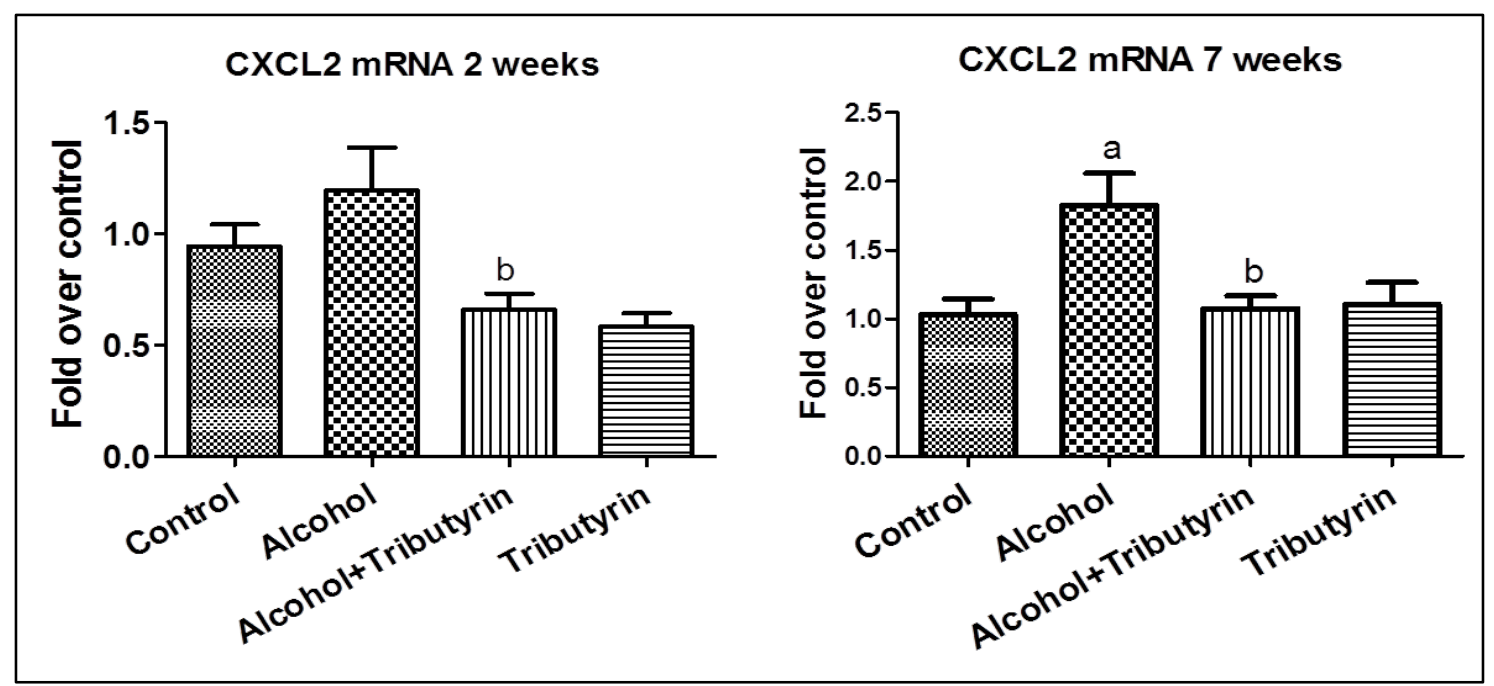

Fig.(25) Chronic alcohol induced chemokine CXCL2 gene expression: CXCL2 mRNA levels were up-regulated in alcohol fed mice and were attenuated by tributyrin treatment. CXCL2 mRNA levels were analyzed by RT-qPCR and normalized to $\beta$-actin mRNA. Data are expressed as mean \pm SEM, $n=5$ animals/per group. $p$ value $a=$ control and $b=$ when compared to alcohol. 
Tributryin administration significantly down-regulates adhesion molecule (ICAM-1) in alcohol-fed mice

It has been shown ICAM-1 plays an important role in alcohol-induced liver injury by facilitating adhesion of leukocytes such as neutrophils [78]. In our chronic alcohol feeding model ICAM-1 mRNA expression was significantly induced compared to pair-fed controls. Tributyrin co-administration significantly decreased alcohol-induced ICAM-1 gene expression in C57BL/6 mice (Fig. 26).

\section{Tributyrin administration decreases neutrophil infiltration in alcohol-fed mice}

Neutrophil infiltration is a hallmark of hepatic inflammation. The above data show that expression of MCP-1 and MIP-2, which are potent chemoattractants for monocytes and/or neutrophils, was unregulated after chronic-alcohol feeding. Therefore, we stained the liver with CAE to scan neutrophils infiltration. As expected, seven weeks of alcohol exposure increased hepatic neutrophils. However, tributyrin administration suppressed neutrophil infiltration as seen by decreased CAE staining (Fig. 27). Neutrophil infiltration was further confirmed by MPO activity assay. MPO activity was significantly increased in alcohol-fed mice at 7 weeks of alcohol feeding (Fig. 28). Tributyrin co-administration significantly reduced MPO activity in the liver. This data indicate that tributyrin administration significantly reduces hepatic inflammation in alcohol-fed mice. 


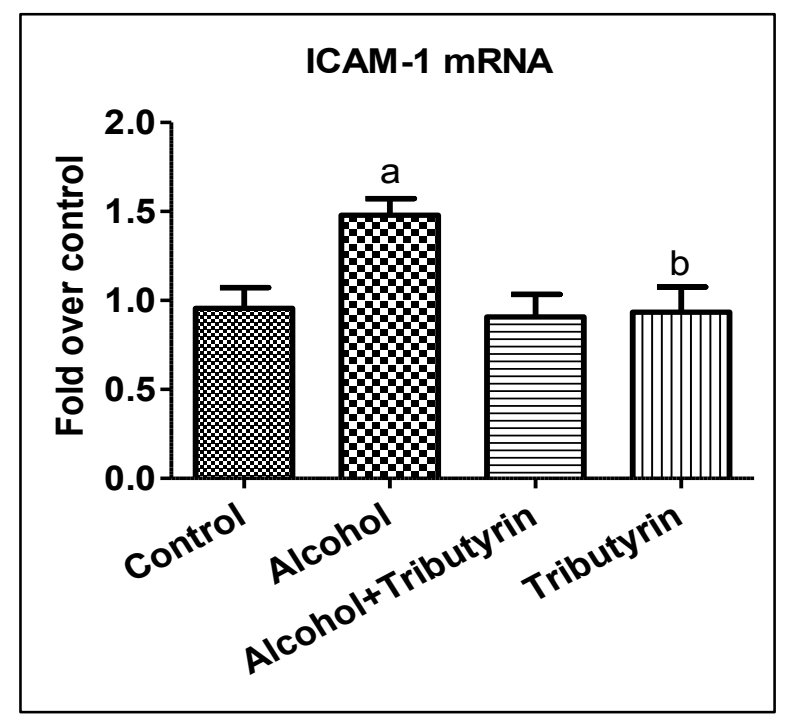

Fig.(26) Chronic alcohol-induced chemokine ICAM-1 gene expression: ICAM-1 mRNA levels were up-regulated in alcohol fed mice and was attenuated by tributyrin treatment. ICAM-1 mRNA levels were analyzed by RT-qPCR and normalized to $\beta$-actin mRNA. Data are expressed as mean \pm SEM, $n=5$ animals/per group. $a=$ control and $b=$ when compared to alcohol. 

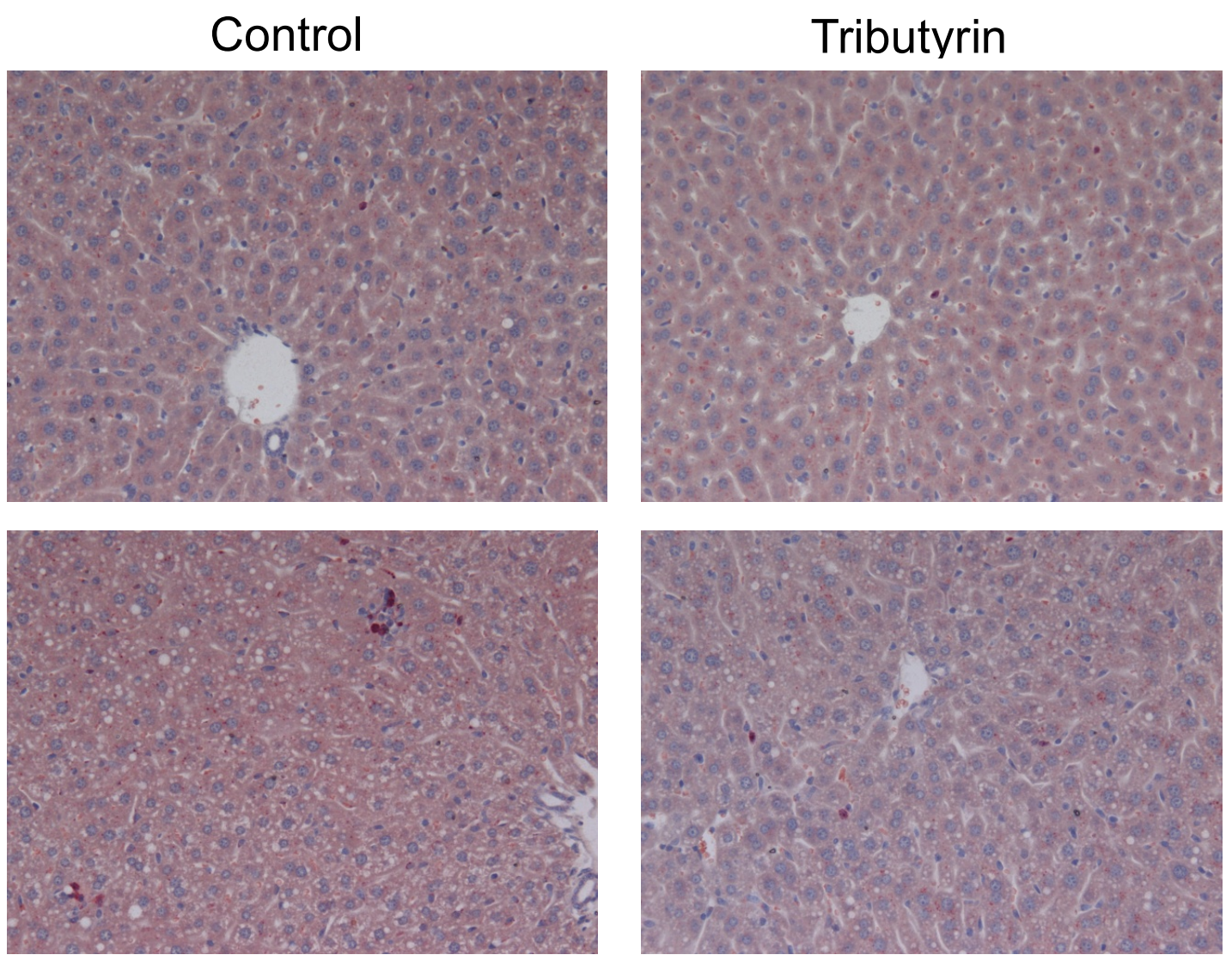

Alcohol

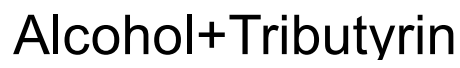

Fig.(27) Effect of tributyrin administration on liver histopathology in mice chronically fed alcohol for 7 weeks. Representative micrographs are shown depicting CAE staining of the liver sections revealing neutrophil infiltration in the alcohol fed mice livers 


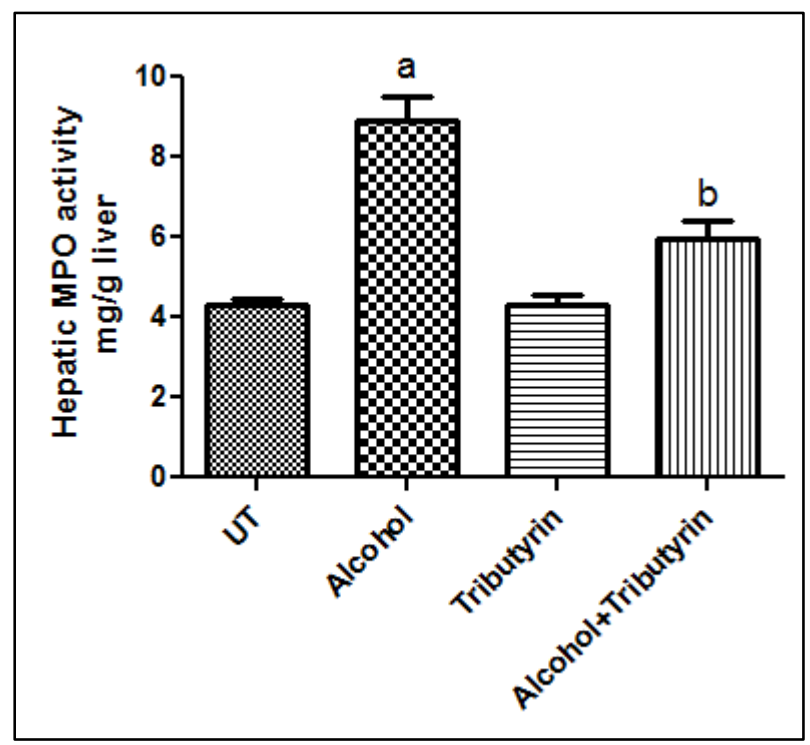

Fig.(28) Hepatic MPO activity in response to alcohol and tributyrin: Hepatic MPO activity in mice was significantly elevated in 7 weeks of alcohol feeding and attenuated by tributyrin treatment. Data are expressed as mean \pm SEM, $\mathrm{n}=4$ to 6 animals/per group. $\mathrm{a}=$ compared to control, $\mathrm{b}=$ compared to alcohol. 
Administration of Tributyrin attenuated Aspartate Transaminase (AST) and Alanine Transaminase (ALT) in alcohol-fed mice

Serum ALT and AST levels are the hallmark of liver injury. It has been well documented that chronic as well as binge alcohol consumption increases AST and ALT levels in the blood indicating liver injury. Hepatic steatosis and inflammation predisposes the liver to cause injury. Hence we measured serum AST and ALT levels in these mice and as expected AST and ALT levels were significantly elevated compared to pair-fed control mice. Co-administration of tributyrin along with alcohol markedly suppressed AST and ALT in mice. This data demonstrates that along with markedly reducing alcohol-induced inflammatory responses in the liver, tributyrin administration attenuated hepatocyte damage and liver injury in chronic alcohol fed mice (Fig. 29). 


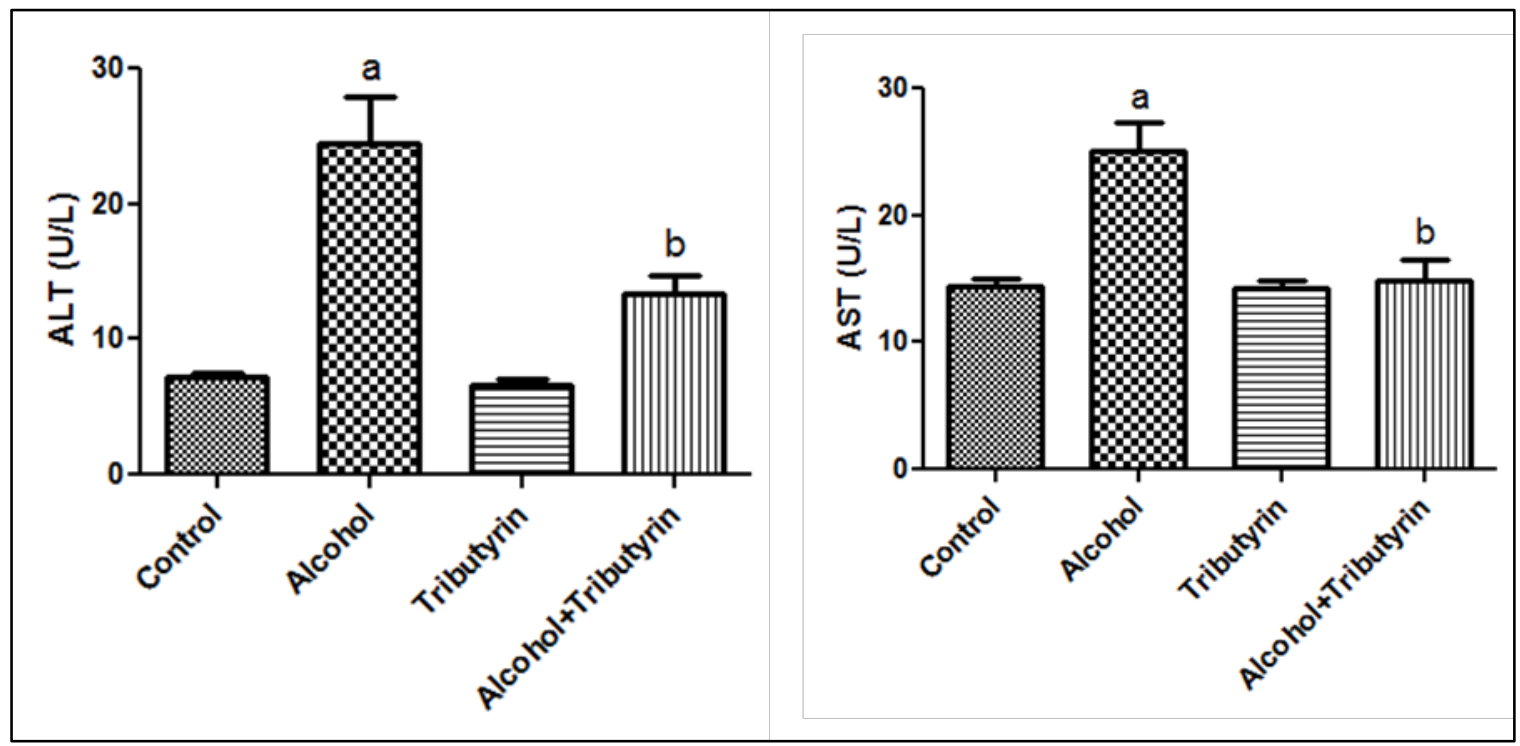

Fig.(29) Effect of tributyrin administration on liver injury in mice chronically fed alcohol for 7 weeks: ALT and AST activity was significantly elevated in 7 weeks of alcohol feeding mice and markedly attenuated by tributyrin treatment. Data are expressed as mean \pm SEM, $n=4$ to 6 animals/per group. $a=$ compared to control, $b=$ compared to alcohol. 


\section{DISCUSSION}

Understanding the pathophysiology and the mechanisms of chronic alcohol-mediated steatosis and steatohepatitis has led to the advancement of new preventive and beneficial strategies in treating alcoholic liver disease. Recent studies have shown that alcohol decreases short-chain fatty acids (SCFAs) [153]. SCFAs, predominantly acetate, propionate and butyrate, are the end-products of anaerobic bacterial fermentation of carbohydrates in the colon. SCFAs, especially butyrate, play important roles in the biology of colonocytes, by acting as the principal energy source and maintaining the integrity of the intestinal environment $[122,154]$. Tributyrin is a butyrate prodrug, and oral administration of tributyrin has been shown to increase butyrate concentration in the hepato-portal circulation [155]. Hence the present study examined the role of tributyrin supplementation in the prevention of alcohol-induced hepatic steatosis, inflammation and injury.

Alcohol consumption causes gut microbial dysbiosis and affect intestinal tight junction protein expression leading to systemic endotoxemia [156]. Since tributyrin is metabolized to butyrate by the intestinal bacteria and pancreatic lipases and has been shown to have protective effects on gut barrier integrity we initially investigated the role of orally administered tributyrin on intestinal ileum tight junction. In accordance to the previous studies [156], tributyrin supplementation prevented the loss of alcohol-induced TJ proteins. Previous 
studies have shown that butyrate treatment improves TJ protein expression by AMPK [157]. Chronic alcohol exposure can decrease the phosphorylation of AMPK leading to its inactivation. Butyrate could be increasing phosphorylation of AMPK during chronic alcohol exposure. This could be a possible mechanism by which butyrate improves the alcohol-mediated suppression of TJ-proteins. In accordance to the literature, alcohol increased systemic endotoxin and was significantly attenuated by tributyrin co-administration.

Hepatic steatosis predisposes the liver to second hits and injuries allowing the progression to severe forms of disease. Alterations in the gut-liver axis are known to drive liver damage. Importantly, it has been shown that oral administration of tributyrin increases the concentration of butyrate in the hepatoportal vein exerting its beneficial effects on the liver [155]. Since we observed tributyrin protection on gut associated changes, we further examined the effect of tributyrin on liver. Immunohistochemical staining and biochemical parameters of hepatic steatosis such as hepatic triglycerides and free fatty acids were significantly reduced by tributyrin. Hepatic triglyceride accumulation and steatosis can occur as a consequence of increased fatty acid synthesis as well as decreased $\beta$-oxidation of fatty acids. Recent studies have shown that alcohol consumption affects both these pathways in hepatic steatosis $[7,158,159]$. Previous studies from our lab have shown that a decrease in CPT-1 plays a critical role in binge alcohol-induced hepatic steatosis [17, 145]. Notably, in the present study Tributyrin/butyrate treatment not only reversed CPT-1 gene expression in chronic alcohol-fed mice and primary hepatocytes but also 
augmented its baseline expression. Because CPT-1 catalyzes a rate limiting step in mitochondrial free fatty acid $\beta$-oxidation, upregulation by tributyrin/butyrate is a significant contributory factor in preventing the development of chronic alcoholinduced liver steatosis. Hence we further investigated the mechanisms underlying the therapeutic effects of butyrate on CPT-1 gene expression by alcohol.

PGC-1 is shown to be a critical co-activator in regulating CPT-1 gene expression [160]. Importantly, it has been reported that PGC-1deficiency leads to the development of hepatic steatosis whereas overexpression of PGC-1 alone is sufficient to induce CPT-1 transcription. Butyrate treatment robustly induced PGC-1 gene expression in the alcohol treated cells indicating that increase in PGC-1 could be a major player in tributyrin mediated up-regulation of CPT-1. In the context of CPT- 1 transcriptional activation, PGC-1 acts as a co-activator and interacts with transcription factors such as PPAR- $\alpha, \mathrm{HNF} 4 \alpha$ increasing promoter histone acetylation [42][35][34]. Interestingly, alcohol treatment was observed to decrease PGC-1 recruitment only at the HNF4 $\alpha$ binding region (DR-1 site)

(fig.15) with minimal to no effect at the PPAR- $\alpha$ binding site (PPRE site) (data not shown). These data indicate that butyrate mediated transcriptional activation of CPT-1 occurs in a PPAR- $\alpha$ independent manner. her paper) - A significant component of butyrate's biological activity is its ability to inhibit HDACs. The role of butyrate as a HDAC inhibitor was evaluated on acetylation state of the CPT-1 and PGC-1 promoter. Particularly, the states of $\mathrm{H} 3 \mathrm{~K} 9 \mathrm{Ac}$ were assessed since this histone modification plays a key role in establishing transcriptionally 
permissive chromatin states. Specifically, H3K9Ac actively neutralizes the basic charge of the affected lysine and ultimately leads to chromatin de-condensation, making the DNA more accessible to transcription factors and RNA polymerases leading to gene transcription. HDAC inhibitory property of butyrate increased the H3K9 acetylation in the alcohol treated cells at TSS site on the CPT-1 and PGC1 promoter. The data obtained strongly supports that the changes in PGC-1 and CPT-1 promoter H3K9Ac levels is achieved by the butyrate HDAC inhibitory function. Additionally, the data support the notion that butyrate mediated increase in $\mathrm{H} 3 \mathrm{~K} 9 \mathrm{Ac}$ results into active transcription as seen by up regulation in CPT-1 and PGC-1 mRNA levels.

The states of CPT-1 promoter H3K9Ac can be significantly enhanced by PGC-1 co-activator function. PGC-1 is known to interact and recruit HATS such as SC-1 and p300 [147, 148]. Besides PGC-1, p300 HAT can also be recruited by the TF SP-1 which plays a role in maintaining the basal and inducible CPT-1 gene expression [150]. P300 is a well-known histone acetyl transferease (HAT) and co-activator that plays a pivotal role in the transcription regulation of gene expression [161, 162]. Indeed, commensurate with the butyrate mediated increase in PGC-1 binding and H3K9Ac levels at the CPT-1 promoter, there was an increase in the recruitment of $p 300$. This reflected by the transcriptionally permissive state of the CPT-1 promoter. This was shown by an increase in the assembly of the pre-initiation complex represented by increased Sp-1 binding and RNA-polymerase II. It is noteworthy that Sp-1 binds and recruits p300 HAT and further influence the transcriptional state. According it is possible increase 
sp-1 binding would further contribute to a net increase in the histone acetylation state of the promoter ultimately contributing to transcriptional activation of CPT-1 gene.

Overall, this data confirms the role of butyrate as an HDAC inhibitor by increasing the net histone acetylation state of the CPT-1 promoter thereby recruiting co-activators PGC-1 and p300 along with TFs and RNA-Pol II, engaging the transcription machinery required for CPT-1 gene expression.

Further, after confirming the role of butyrate in alcohol-induced hepatic steatosis we investigated the effect of tributyrin/butyrate on alcohol-induced hepatic inflammation. TNF- $\alpha$, a key cytokine involved in the development of alcoholic liver disease was significantly down-regulated by tributyrin administration as early as 2 weeks indicating that butyrate suppresses initial inflammation caused due to alcohol consumption. Additionally, chemokines such CCL-2/MCP-1 has been shown to be significant factors in various chronic inflammatory diseases [163]. Recent studies have shown that MCP-1 regulation shares a common link between inflammatory cytokines and lipid metabolism in alcoholic liver disease [93]. In our animal model we have shown that CCL-2 was induced by 2 weeks of alcohol-feeding and its high expression was maintained up to 7 weeks. This indicates that CCL-2 regulation is involved in sustained inflammation. Moreover, alcohol primed the hepatocytes and increases their sensitivity to TNF- $\alpha$ inducible CCL-2 expression. Butyrate significantly downregulated CCL-2 induction indicating that butyrate can directly act on the hepatocytes to suppress inflammatory chemokines. Potentially, butyrate being as 
HDAC inhibitor, could also be affecting acetylation of non-histone proteins including certain transcription factors responsible for the induction of CCL-2. $\mathrm{NF \kappa B}$ is known to play a central role in immune and inflammatory responses and is involved in transcriptional regulation of many chemokines and cytokines including TNF- $\alpha$. Suppression of LPS inducible TNF- $\alpha$ by butyrate in RAW cells indicated that butyrate inhibits pro-inflammatory cytokine production in macrophages. Hence we speculate that butyrate inhibits NFKB activation via affecting its acetylation state and/or promoting $\mathrm{I}_{\kappa} \mathrm{B} \alpha$ degradation [133] leading to resultant down-regulation of CCL-2 gene expression. In addition to $\mathrm{NF} \kappa \mathrm{B}, \mathrm{CCL}-2$ is also regulated by Activator protein-1. Hence butyrate could suppress CCL-2 expression by decreasing the DNA binding activity of NFKB and AP-1 onto the CCL-2 promoter. Immunohistochemical analysis showed that butyrate suppressed alcohol-induced neutrophil infiltration and macrophage activation as shown by F4/80+ cells. Finally liver injury markers, AST and ALT were significantly down-regulated by triburyrin administration indicating that tributyrin prevented alcohol-induced early liver injury at 2 weeks as well as sustained injury at 7 weeks.

Overall this data indicates that tribtyrin/butyrate through HDAC inhibition significantly prevented alcohol-induced hepatic steatosis, inflammation and injury. 


\section{SUMMARY AND CONCLUSIONS}

Work done in this thesis examines the role and the underlying molecular mechanisms of Tributyrin in the attenuation of Alcoholic liver disease in mice. Decades of research have shown that chronic alcohol abuse is associated with alcoholic liver disease (ALD). Clinically, stages of alcoholic liver disease include fatty liver, steatohepatitis followed by fibrosis and cirrhosis leading to hepatocellular carcinoma. Hepatic steatosis is transient and temporary. If not treated, it can progress into steatohepatitis leading to the development of alcoholic liver disease. There is no FDA-approved therapy for any stage of alcoholic liver disease. Hence it is of prime importance to understand the mechanisms of Alcoholic liver disease which would lead us to a better understanding of therapeutic intervention and further developing novel strategies/therapies in the treatment of ALD. Hence in this project, we have investigated the effects of Tributyrin supplementation; a pro-drug of butyrate in attenuating alcohol induced hepatic steatosis, inflammation injury in mice. Previous studies have shown that increase in the butyrate levels in the hepatoportal circulation prevents LPS-induced liver injury in rats. The hypothesis of this project is that oral administration of tributryin targets the 'gut-liver' axis and significantly blocks the progression of alcoholic liver disease (ALD).

We have used a well-established animal model of alcoholic liver disease to examine the effects of $\mathrm{Tb}$ oral administration on ethanol-induced changes in 
intestinal permeability and hepatic steatosis, inflammation and injury. 8-10-week old C57BL/6 male mice were pair-fed the Lieber-DeCarli liquid diet containing alcohol or isocaloric maltose dextrin for a period of 7 weeks. Tributyrin was administered to a sub-group of alcohol-fed animals by oral gavage to assess its effects. In order to monitor the temporal changes in the progression of ALD, these animals were sacrificed at 2, 4 and 7 weeks.

Initial analysis revealed Tributyrin attenuated the ethanol-induced gut barrier dysfunction, as shown by the significant reduction in endotoxemia. Mechanisms of chronic alcohol-induced gut-barrier dysfunction were examined by the immune-histochemical analyses of the ileum section of mice chronically fed alcohol. Specifically, alcohol induced a robust down-regulation of intestinal tight junction proteins ZO-1 and occludin which was markedly attenuated by Tributyrin. Additionally, Tributyrin treatment significantly decreased alcohol induced hepatic steatosis as well as hepatic triglycerides. Tributyrin prevented ethanol-induced down-regulation of CPT-1 gene expression (RT-PCR), a key enzyme in fatty acid $\beta$-oxidation. Tributyrin being a prodrug is metabolized to butyrate by pancreatic lipases. Butyrate is a HDAC inhibitor. To understand the regulation of CPT-1 gene expression under pathogenic conditions experiments were performed using primary rat hepatocytes. Hence acetylation at CPT-1 promoter was investigated. Acetylation of histone 3 lysine 9 (H3K9), a critical feature of the active promoter state was suppressed by alcohol treatment in hepatocytes reversed by butyrate. PGC-1 a critical co-activator of CPT-1 and Sp1, transcription factor binding to DNA was robustly increased by butyrate. 
Additionally, p300 a histone acetyl transferase was shown to be recruited at the DR-1 binding site as well as TSS site on the CPT-1 promoter region indicating an increase in the net acetylation. Hence butyrate being an HDAC inhibitor increases the net acetylation state at the promoter region of CPT-1 gene leading to its transcriptional activation and regulation.

Hepatic inflammation was markedly decreased in Tributyrin administered alcoholic mice seen by liver immunohistochemistry staining. Myeloperoxidase activity, a quantifiable measure of inflammation was significantly dampened by Tributyrin. Tributyrin also attenuated the ethanol induced hepatic expression of the critical inflammatory cytokine, TNF- $\alpha$. Liver injury markers such as AST and ALT were significantly attenuated at the end of 7 weeks in Tributyrin treated mice. Moreover, Tributyrin also prevented the ethanol-induced expression of chemo-attractant chemokines ( $\mathrm{CXCl} 2, \mathrm{CCl} 2$ and ICAM-1), and neutrophil recruitment that are critical for the development of ALD. Future studies are aimed to understand the underlying mechanisms of how butyrate protects the gut and the liver in alcohol fed animals.

Overall, the present work demonstrates that tributyrin may be useful in preventing the ethanol-induced gut barrier dysfunction and associated pathologic hepatic changes, and may prove to be a useful therapy for the prevention/treatment for ALD. 


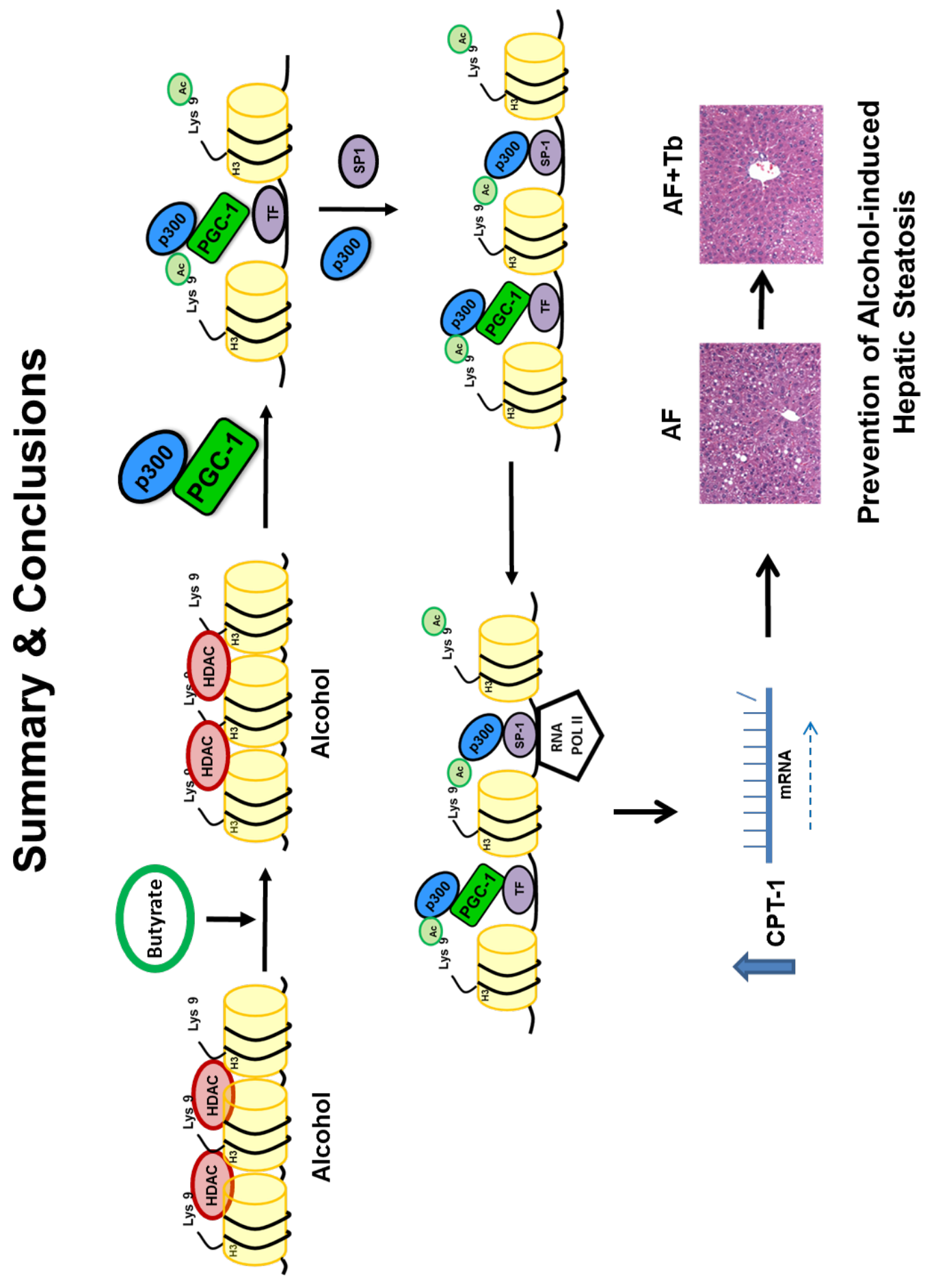




\section{SECTION II}

\section{DETERMINE THE MECHANISMS OF HIV-ANTIRETROVIRAL THERAPY INDUCED LIVER INJURY}




\section{INTRODUCTION}

\section{HIVIAIDS- An overview}

HIV (Human Immunodeficiency Virus) infection is considered pandemic by the World Health Organization (WHO), claiming more than 25 million lives over the past three decades. Thirty four million people are affected worldwide. Centre for Disease Care (CDC) estimates 1.2 million people in the United States (US) are living with HIV infection (www.cdc.gov). One in five (20\%) of those people are unaware of their infection. By race, African Americans face the most severe HIV burden. Men having sex with men (MSM), particularly the young, are most severely affected. HIV infection leads to AIDS (Acquired Immunodeficiency Syndrome). AIDS was first recognized in the United States in the summer of 1981, when the U.S. (CDC) reported the unexplained occurrence of Pneumocystis jiroveci (formerly P. carinii) pneumonia in five previously healthy homosexual men in Los Angeles. In 1983, human immunodeficiency virus (HIV) was isolated from a patient with lymphadenopathy, and by 1984 it was demonstrated clearly to be the causative agent of AIDS. Since the epidemic began, an estimated 1,108,611 people in the US have been diagnosed with AIDS (www.cdc.gov). 


\section{HIV and HAART}

In order to prevent or delay resistance development in HIV infected patients, physicians are using a regimen which consists of a combination of several types of antiretroviral drugs that act on different sites on a retro virus and also target different stages in the lifecycle of the virus. Such treatments are called highly active antiretroviral therapy (HAART) [164]. The development of multi-drug combination therapy for treatment of HIV disease is considered one of the great success stories of modern medicine. In a period of approximately ten years, the death rate from HIV disease was reduced by 50 to $80 \%$ [165]. HAART as treatment for HIV infection has greatly improved mortality and morbidity for adults and children living with HIV around the world [166, 167]. HAART is the only treatment available to suppress the viral load. Currently used HAART medication include nucleoside reverse transcriptaseitnhibitors (NRTIs) (zidovudine, didanosine, abacavir), non-nucleoside reverse transcriptase inhibitors (NNRTI) (nevirapine, etravirine), protease inhibitors (PI) (indinavir, ritonavir, lopinavir, atazanavir), Fusion inhibitors (Enfuvirtide), Integrase Inhibitors (Raltegravir), and CCR5 inhibitors all of which act on different sites on HIV facilitating a decrease in viral load. Current treatment guidelines strongly recommends the use of tripledrug regimens which consists of two nucleoside reverse transcriptase inhibitors (NRTIs) with either a protease inhibitor $(\mathrm{PI})$ or a non-nucleoside reverse transcriptase inhibitor (NNRTIs) as a first line option for the initial treatment of early and advance disease progression [95]. Since the introduction of highly active antiretroviral therapy (HAART) in the mid-1990s, the life expectancy of 
patients with HIV has increased significantly $[168,169]$. Although HAART has been widely used in the treatment of HIV, HIV infected patients have experienced complications associated with HAART, which cannot be ignored. Antiretroviral toxicity, resistance, and adherence rank high on the list of problems that must be overcome if all, or at least the vast majorities, of HIV-infected patients are to gain the long-term benefits associated with HAART [170]. Among these problems, toxicity, real or perceived, is a major reason for patients either refusing or prematurely discontinuing HAART [171]. The drop in mortality and morbidity due to opportunistic infection has been accompanied by a concomitant increase in HAART-related hepatotoxicity which caused discontinuation of HAART in increasing numbers of patients. HAART hepatotoxicity, including lactic acidosis, hepatic steatosis and lipodystrophy, occurs in approximately $10 \%$ of patients and is higher in those with underlying liver disease [172].

Nucleoside Reverse Transcriptase Inhibotors (NRTI)- Nucleoside/nucleotide reverse transcriptase inhibitors (NRTIs), inhibit the formation of viral DNA by incorporating into the newly formed DNA molecules, thereby preventing further elongation of those molecules [173].

Protease Inhibitor (PI) - Protease inhibitors block the activity of the protease enzyme, which HIV uses to degrade large polyproteins into the smaller pieces required for assembly of new viral particles. All PIs are metabolized in the liver by CYP3A isoenzymes; consequently their metabolic rates may be altered in the presence of CYP inducers or inhibitors. Co-administration of Pls with a potent CYP3A inducer may lead to suboptimal drug concentrations and reduced 
therapeutic effects of the PI. Hence co-administration of Pls with ritonavir (RTV), a potent CYP3A inhibitor will optimally increase PI exposure. [174]. While HIV can still replicate in the presence of protease inhibitors, the resulting virions are immature and unable to infect new cells. PI -based regimen has seen demonstrated to have anti-virologic potency. Unlike NRTI resistance, virologic failure rarely selects for PI-resistance, which confers it with increased efficiency in decreasing the viral load.

\section{Ritonavir and Lopinavir- Protease inhibitors}

Lopinavir is a novel PI developed from ritonavir. It is structurally related to ritonavir with the same mechanism of action. Lopinavir has poor oral bioavailability coupled with its extensive and rapid metabolism in the liver, resulting in a short elimination half-life (t1/2). However, co-administration with subtherapeutic doses of ritonavir improves these pharmacokinetic properties of lopinavir allowing the drug to exert its highly potent antiretroviral activity [175, 176].

\section{Mechanism of action of Protease inhibitors}

Lopinavir is a higly potent inhibitor of HIV-1 protease $[177,178]$. This enzyme is a homodimeric aspartic protease involved in the post-translational processing of viral gag and gag-pol polyprotein products into functional core proteins and viral enzymes [179]. HIV PIs, including lopinavir, prevent cleavage of gag and gag-pol protein precursors in acutely and chronically infected cells, arresting maturation and thereby blocking the infectivity of nascent virions [180, 181]. 


\section{HAART associated hepatotoxicity and liver injury}

HAART causes a variety of adverse effects like cardiovascular diseases, neurological disorders, liver disease, lipodystrophy, non-Hodgkin lymphoma to name a few. Liver disease is recognized as an increasingly important problem for the HIV population, and may be due to a variety of factors including co-infection with viral hepatitis, alcohol abuse, and antiretroviral hepatotoxicity. Liver disease is now a leading cause of death for patients with HIV. In patients co-infected with HIV and hepatitis C, cirrhosis was the underlying cause of death in nearly $50 \%$ [171]. In a more general HIV population, liver disease was the second most common non-HIV-related cause of death, trailing only cancer [182]. The earlier retrospective study showed that the discontinuation of ART due to hepatotoxicity increased from 6\% in 1996 to $31.8 \%$ in $1998-1999$ and was the main cause of death in HIV infected individuals [183]. The severity of liver toxicity ranges from the absence of symptoms to liver decompensation, and the reported incidence of severe liver toxicity after initiating HAART ranges from $2 \%$ to $18 \%$. Hepatotoxicity due to HAART is also common and up to $30 \%$ of patients on HAART experience World Health Organization grade 3 liver enzyme elevations. Hepatotoxicity induced by these agents has been identified on the basis of circulating liver enzymes (ALT, AST, and GGT) in association with increased lactate levels. In more dramatic situations, liver failure has occurred leading to discontinuation of treatment. The study of hepatotoxicity induced by HAART is complicated by the diverse chemical nature of the agents and by the differences between the effects of agents used singly or in combination. Hepatotoxicity from antiretroviral drugs 
has led to adverse patient outcomes either from fulminant hepatic failure, or more commonly, AIDS following discontinuation of HAART. Mechanisms contributing to the adverse effects of HAART induced hepatotoxicity are still largely undetermined. Hence, it is highly relevant to study the underlying mechanisms of HAART-induced hepatotoxicity.

\section{Role of ER-stress in HIV-PI induced liver injury}

Recent evidence suggests that HIV-PIs induce ER-stress and promote liver injury [184-186]. The ER is a critical organelle responsible for proper protein folding, cellular calcium levels, lipid synthesis and the secretory pathway. ER stress is primarily mediated by the ER protein BiP/GRP78 (Glucose regulated protein 78) via three ER sensors ATF6 (activating transcription factor 6), IRE-1 (inositol-requiring enzyme1), and PERK (protein kinase RNA-like endoplasmic reticulum kinase). ER stress comprises both pathologic and adaptive responses; UPR adaptive responses reduce the protein burden by decreased synthesis and increased ER-associated degradation (ERAD) of proteins, and by inducing ER chaperones to enhance folding capacity. If the adaptive/protective reactions are inadequate or if the ER stress is prolonged/extreme, apoptotic cell death ensues via pathways involving activation of JNK (cJun N-terminal kinase) and caspase12, and upregulation of proapoptotic proteins like CHOP (CCAAT/enhancerbinding protein homologous protein)[187]. CHOP also known as growth-arrest DNA damage-inducible gene 153 (GADD153) is a major transcription factor involved in ER-tress mediated apoptosis [188]. 
HIV PIs, individually or in combination were shown to increase ER-stress markers such as active sterol regulatory element-binding proteins (SREBPs), $\mathrm{X}$ box binding protein 1 (Xbp1), activating transcription factor 4 (ATF-4), C/EBP homologous protein (CHOP) and caspase-12, leading to apoptosis in macrophages and rat hepatocyte. Additionally, ER calcium store depletion and lipid dysregulation in the ER by HIV PIs were shown to activate unfolded protein response in the hepatocytes $[184,185,189]$. Co-factors such as alcohol or Hepatitis C or B virus may exacerbate HIV-PIs induced ER-stress and liver injury.

\section{Role of Fas/FasL pathway in drug induced liver injury}

Liver is an important organ for metabolism and elimination of foreign substances. Hence it is targeted for drug toxicity. Excessive hepatocyte cell death has been shown to be the central mechanism of liver damage in conditions such as viral hepatitis; alcoholic steatohepatitis, non-alcoholic steatohepatitis (ASH and NASH) as well as drug induced liver injury (DILI). The Fas/FasL signaling pathway has been implicated in various liver pathologies including Wilson's disease, alcoholic liver disease (ALD), acute fulminant hepatitis, as well as chronic viral hepatitis [190-192]. Fas (CD95) is a death receptor in the tumor necrosis factor receptor (TNFR) family and is expressed in a variety of tissue[193]. Upon binding, the receptor undergoes trimerization and forms the intracellular death-inducing signaling complex (DISC) [193, 194]. This DISC include Fas-associated protein with death domain (FADD). This activates caspases signaling cascade via mitochondria dependent and independent pathways leading to apoptotic cell death [193]. Typically hepatocytes express 
high levels of Fas receptor and respond to FasL signals from activated immune cells; however, under pathologic conditions such as alcoholic cirrhosis, hepatocytes also express high levels of FasL, leading to fratricide and liver injury. Moreover, endotoxin-induced liver injury in mice occurs via the Fas/FasL pathway following induction of Fas by IL-18 from Kupffer cells, and hepatocyte death induced in mice following exposure to anti-Fas antibody (JO-2) is an accepted model of fulminant hepatitis. With regards to HIV-positive individuals, increased expression of FasL is seen in cardiomyocytes, which contributes to AZT-Induced cardiomyopathy [195].

\section{Role of Phosphodiesterase 4 (PDE4)/cAMP in liver injury}

cAMP is a second messenger that plays a critical role in regulating multiple cellular functions and its levels are tightly regulated through biosynthesis by adenyl cylases (ACs) and hydrolysis by phosphodiesterase (PDEs). cAMP exerts its effect through effector molecules protein kinase $A(P K A)$ and Exchange Protein directly Activated by cAMP (EPAC), also known as cAMPGEF. There are 11 different members of the mammalian class I PDE super family, (PDE1-PDE11); within the families there are multiple genes and a large number of PDE splicing variants. Distinct kinetic and functional properties are attributed to PDE families with some specifically hydrolyzing cAMP, some both cAMP and cGMP, and others only cGMP [196, 197]. Of the cAMP specific PDEs, the PDE4 family is widely expressed and is the current therapeutic target for the treatment of inflammatory diseases, such as asthma and chronic obstructive pulmonary disease, as well as depression and cognitive deficit [197, 198]. 
PDE4 accounts for the majority of cAMP hydrolysis activity in cells[199]. PDE4 represents a large and complex family, with 4 genes (PDE4A/B/C/D) encoding over 20 distinct PDE4 isoforms as a consequence of distinct promoter usage and mRNA splicing; notably, these isoforms have similar catalytic activities but distinct cellular functions. PDE4 isoforms are regulated by transcriptional and post-translational mechanisms. Work done in our lab has shown that PDE4 expression is significantly increased in response to alcohol exposure in monocytes/macrophages including hepatic Kupffer cells [200]. Notably, Work done by us and others has shown that regulation of cellular cAMP levels via degradation by phosphodiesterase (PDEs), particularly cAMP-specific

PDE4B plays an essential role in LPS-induced TLR4 signaling and inflammatory cytokine expression by monocytes/ macrophages [201-203](Jin and Conti, 2002; Jin et al., 2005; Gobejishvili et al., 2011). Additionally, in cholestatic liver injury, besides downregulating systemic and hepatic inflammatory cytokines, increases in cAMP have been shown to protect hepatocytes from apoptosis due to several related stimuli, including bile acids, LPS, Fas, and TNF- $\alpha$ [204-207].

In another study done by our lab bile duct ligation mediated liver injury and apoptosis was significantly attenuated by PDE4 inhibition as assessed by reduced liver enzyme levels, decreased TUNEL staining, and PARP-1 cleavage. Hence, in addition to its anti-inflammatory effects, PDE4 inhibition, which leads to increased cellular cAMP levels, could also attenuate hepatocyte death. Indeed, cAMP has been shown to protect hepatocytes from bile acid-induced death by 
affecting protein kinase $\mathrm{A}(\mathrm{PKA})$ and $\mathrm{CAMP}$-guanine exchange factor-mediated signaling [206-208]. 


\section{OVERALL HYPOTHESIS}

We hypothesized that Protease inhibitor induced PDE4 expression and altered cAMP metabolism plays a major role in the induction of hepatocyte ER-stress, apoptotic gene expression and injury. (Fig. 30)

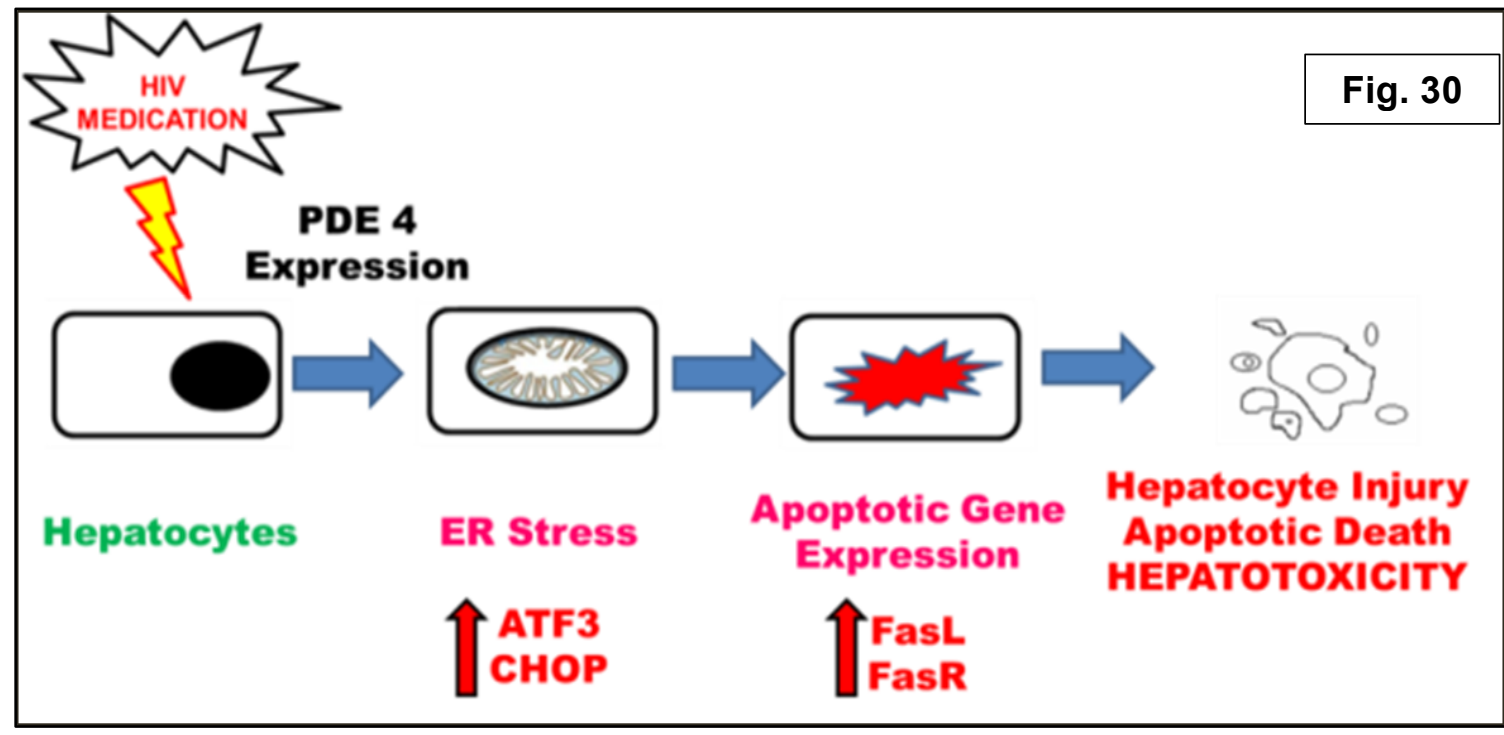




\section{SIGNIFICANCE \& CLINICAL RELEVANCE}

In the United States, approximately 2000 cases of acute liver failure occur annually and drugs account for over $50 \%$ of them (39\% are due to acetaminophen, $13 \%$ are idiosyncratic reactions due to other medications).

Drugs account for $2-5 \%$ of cases of patients hospitalized with jaundice and approximately $10 \%$ of all cases of acute hepatitis. HAART has been associated with increase in morbidity and mortality due to HAART-related hepatotoxicity and finally discontinuation of therapy. This is especially true in patients who are coinfected or have other cofactors such as alcohol abuse. The mechanisms for HAART hepatotoxicity have received very limited attention. There is no wellaccepted therapeutic intervention except for abstinence and HAART discontinuation. Since liver is the first organ exposed and metabolized by the drugs it becomes highly relevant to understand the mechanism of drug toxicity. Findings from this project could provide important mechanisms by which HAART$\mathrm{PI}$ induced pathology causes cell death in hepatocyte often observed by prolonged drug intake in HAART medication. Moreover, the findings from this study would be significant in the development of successful preventive and therapeutic strategies in most-at-risk populations. Finally, the proposed studies will focus on direct hepatotoxicity due to HAART by the combinations of ritonavir and lopinavir (HAART PIs drugs), defining the disease mechanisms and generate therapeutic targets. 


\section{MATERIALS AND METHODS}

\section{Reagents}

PI drugs Ritonavir and Lopinavir were purchased from Selleckchem (Houston TX). Rolipram was purchased from Enzo Life sciences (Farmingdale, NY). H-89 and Dimethyl sulfoxide (DMSO) were purchased from Sigma-Aldrich (St. Louis, MO). Horse serum was purchased from ATCC. Fetal bovine serum was purchased from Atlanta Biologicals (Norcross, GA). DMEM, penicillin, streptomycin and TRIzol ${ }^{\circ}$ were obtained from Invitrogen (Carlsbad, CA). All RTPCR reagents were purchased from Applied Biosystems (Foster City, CA). MTT (Thiazolyl Blue Tetrazolium Bromide) was obtained from Sigma-Aldrich (St. Louis, MO).

\section{Cell Culture}

H4IIEC3 rat hepatoma cells (ATCC, Rockville, MO) were cultured in Dulbecco's modified eagle medium (DMEM) supplemented with 10\% Horse Serum and 5\% fetal bovine serum (FBS), $10 \mathrm{U} / \mathrm{ml}$ penicillin, and 10 g/ml streptomycin. Cells were maintained in an incubator at $37^{\circ} \mathrm{C}$ with humidified $5 \% \mathrm{CO}$. Cells were plated and incubated overnight before treatment, in all experiments.

\section{Cell Survival Assay}

The MTT assay was used to assess cell survival. For the MTT assay, cells were plated in 96 well microplates, incubated overnight and treated with the treatments 
mentioned in results for 24 hours. Treatments were then removed and MTT made in fresh DMEM $(1 \mathrm{mg} / \mathrm{ml})$ was added to the wells $(100 \mu \mathrm{l} /$ well $)$. After incubation at $37^{\circ} \mathrm{C}$ with humidified $5 \%$ CO2 for $1-3$ hours, DMSO (200 $\mu \mathrm{l} /$ well) was added. Cell viability was quantified colorimetrically by measuring $0 . D$. at 570 $\mathrm{nm}$.

\section{RNA Isolation and Real-Time PCR Analysis}

Total RNAs were isolated from liver tissue and cell cultures using TRIzol reagent (Invitrogen, Carlsbad, CA) and treated with DNase I to remove any contaminating genomic DNA (RQ1 RNase-Free DNase; Promega). For RT-qPCR, the firststrand cDNA was synthesized using qScript cDNA SuperMix (Quanta Biosciences, Inc., Gaithersburg, MD). qRT-PCR was performed in triplicate with an ABI Prism 7500 sequence detection system and Perfecta SYBR Green FastMix, Low ROX reagents (Quanta Biosciences). The RT conditions were 10 mins at $25^{\circ} \mathrm{C}, 30 \mathrm{mins}$ at $48^{\circ} \mathrm{C}$ and 5 mins at $85^{\circ} \mathrm{C}$. Each sample was run in duplicate reactions. Reverse transcriptase polymerase chain reaction (RT-PCR) assays were used to assess mRNA levels of desired genes in liver tissues as well as hepatocytes H4IIEC3 cells. The primers amplifying the genes examined were designed in the lab and obtained from Intergrated DNA Technologies, Inc (Coralville, IA). The parameter threshold cycle (Ct) was defined as the fraction cycle number at which the fluorescence passed the threshold. The relative gene expression was analyzed using the 2-AACt method by normalizing with $\beta$-actin mRNA gene expression in all the experiments and is presented as fold change over Untreated which is set at 1. 


\section{Western Blot analysis}

Total cellular extracts were prepared by lysing the cells in RIPA lysis buffer (25mM Tris/HCL pH 7.5, 150mM NaCl, 1\% NP-40, 0.1\% SDS, $1 \mathrm{mM} \mathrm{DTT,} 10 \%$ glycerol, $1 x$ protease inhibitor cocktail, $0.5 \%$ sodium deoxycholate, $1 \mathrm{mM} \mathrm{Na}_{2} \mathrm{VO}_{3}$ and $10 \mathrm{mM} \mathrm{NaF}$ ). Protein samples were prepared in SDS loading buffer and proteins were separated by electrophoresis on $10 \%$ polyacrylamide gels and transferred onto a PVDF membrane. The blots were blocked with $5 \%(\mathrm{w} / \mathrm{v})$ nonfat dry milk constituted in 1x TBS-T (Tris buffered saline, 10mM Tris HCL pH 8.0, $150 \mathrm{mM} \mathrm{NaCl}, 0.1 \%$ Tween 20) for 1 hour at room temperature. Membranes were incubated at $4^{\circ} \mathrm{C}$ overnight with primary antibodies directed against proteinof-interest in $5 \%(\mathrm{w} / \mathrm{v})$ nonfat dry milk in $1 \mathrm{x}$ TBS-T. After overnight incubation in primary antibod, blots were washed and incubated with appropriate horseradish peroxidase-conjugated secondary antibodies for an hour. Immunoreactive bands were visualized using the enhanced chemiluminescence light $(E C L)$ detection reagents (Amersham, Arlington Heights, IL). Data shown are representative of 2 separate experiments showing similar results. Primary antibodies for AFT3, 4 CHOP, PDE4A, B D and FasL were purchased from cell signaling. $\beta$-actin antibody was obtained from Sigma-Aldrich (St. Louis, MO). Secondary antibodies against mouse and rabbit were obtained from Santacruz Biotechnologies, Inc. (Santacruz, CA).

\section{Statistical Analysis}

Data are presented as means \pm SD for the indicated number of independently performed experiments. Student's t-test and one-way ANOVA with Bonferroni 
multiple comparison test were used for the determination of statistical significance. $P<0.05$ was considered significant.

mRNA PCR Primer Sequences

\begin{tabular}{|l|l|}
\hline primername & Sequence \\
\hline rATF3_F1 & GGGCCACCTCAGACTTGGTGACT \\
\hline rATF3_R1 & CATCGGATGTCCTCTGCGCTGG \\
\hline rATF4_F1 & AAGCCATGGCGCTCTTCACGA \\
\hline rATF4_R1 & AGTCCCCCGCCAACACTTCG \\
\hline & \\
\hline rDDIT3_GADD_CHOP_F1 & TGTTGAAGATGAGCGGGTGGCAG \\
\hline rDDIT3_GADD_CHOP_R1 & TGGACCGGTTTCTCTCTCCTCAGGT \\
\hline & \\
\hline r.n_FasL_F1 & CTCTGGTTGGAATGGGGTTA \\
\hline r.n_FasL_R1 & TTGGTTTCAGAGGGTGTGCT \\
\hline & \\
\hline r.n._Fas_F2 & CATTTTGCTGTCAACCGTGT \\
\hline r.n._Fas_R2 & CGTGTACTCCTCCCCTTCTG \\
\hline & \\
\hline rActb_F2 & CAGCTGAGAGGGAAATCGTG \\
\hline rActb_R2 & CTCCAGGGAGGAAGAGGATG \\
\hline & \\
\hline rPde4acm1_F & ATGGCTGAGTTCTTCCAGCA \\
\hline rPde4acm1_R & CCATGTCTCCCACAATGGAT \\
\hline & \\
\hline rPde4bcm2_F & GGAGTTGTATCGGCAATGGA \\
\hline rPde4bcm2_R & CAATGAAACCAACCTGGGACT \\
\hline & \\
\hline rPde4dcm1_F & TGGATCATCCTGGTGTGTCA \\
\hline rPde4dcm1_R & TTAAAGCCCACAGCCAAATG \\
\hline
\end{tabular}




\section{RESULTS}

In this study we investigated the mechanisms involved in HIV-PI induced hepatotoxicity. We specifically examined the role of ER-stress and apoptotic Fas/Fas expression in the HIV-PI mediated hepatocyte death. Importantly, we determined the pathogenic role of PDE4 /CAMP metabolism in the development of HIV-PI-induced hepatotoxicity.

\section{HIV-protease inhibitors induces PDE4 expression in H4IIE rat hepatoma cell}

\section{line}

Work done by our research group has demonstrated that PDE4 expression leading to a decline in cellular cAMP levels plays a major pathogenic role in the development of alcohol induced liver disease and cholestasis liver injury and fibrosis [200, 209]. Hence we investigated the involvement of PDE4 enzymes in Pl-induced hepatotoxicity. mRNA gene expression was carried out to profile the expression of PDE4 sub-families A,B and D in H4IIE hepatocytes treated with Ritanovir (Rit) and Lopinavir (Lop) $(20 \mu \mathrm{M})$ each singly and in combination. Differential pattern of the gene expression in PDE4 sub-family member was observed (Fig.31). Specifically, PDE4B gene expression was induced as early as 3hrs by the combination treatment of Rit and Lop. PDE4B expression was observed to go back to baseline by 6 hours. In contrast PDE4A and PDE4D mRNA expression was only observed to be significantly induced by the PI combination at $24 \mathrm{hrs}$ in the combinatorial treatment of Ritanovir and Lopinavir 
$(20 \mu \mathrm{M})$. Further, in correspondent to the changes in mRNA expression the combination of PI drugs showed an increase in PDE4B and PDE4D protein expression as shown by western blot analysis (Fig31); interestingly PDE4A protein expression did not show an appreciable change in the cells treated with Pls together at $24 \mathrm{hours}$. Since western blot data was done only at $24 \mathrm{hrs}$, possibility of PDE4A induction by PI drugs at early time-points or time-points after $24 \mathrm{hrs}$ cannot be ruled out. Overall this data suggests that PIs in combination induce PDE4 expression in hepatocytes that can affect cAMP metabolism.

\section{Inhibition of PDE4 led to a significant increase in the survival of HIV-PI treated H4IIE rat hepatocytes}

Cell viability was measured in hepatocytes treated with individual drugs (Ritanovir 20uM or Lopinavir 20uM) and combination (Ritanovir and Lopinavir 20uM each) for $24 \mathrm{hrs}$. The effect of PDE4 inhibition was examined by adding rolipram along with Rit+Lop combined treatment. Individual drugs Ritanovir and Lopinavir decreased cell viability by $30 \%$ whereas combination of drugs $(R+L 20 u M)$ further significantly decreased it by $55 \%$ as compared to untreated (Fig.32). However addition of rolipram along with (Rit+Lop) prevented the loss of hepatocyte and increased survival upto 65\% (Fig.32). This data indicates that (Rit and Lop) singly and in combination induces cell death in hepatocytes whereas inhibition of PDE4 by the addition of rolipram significantly increased the cell survival in H4IIE cell line. 
3 hours
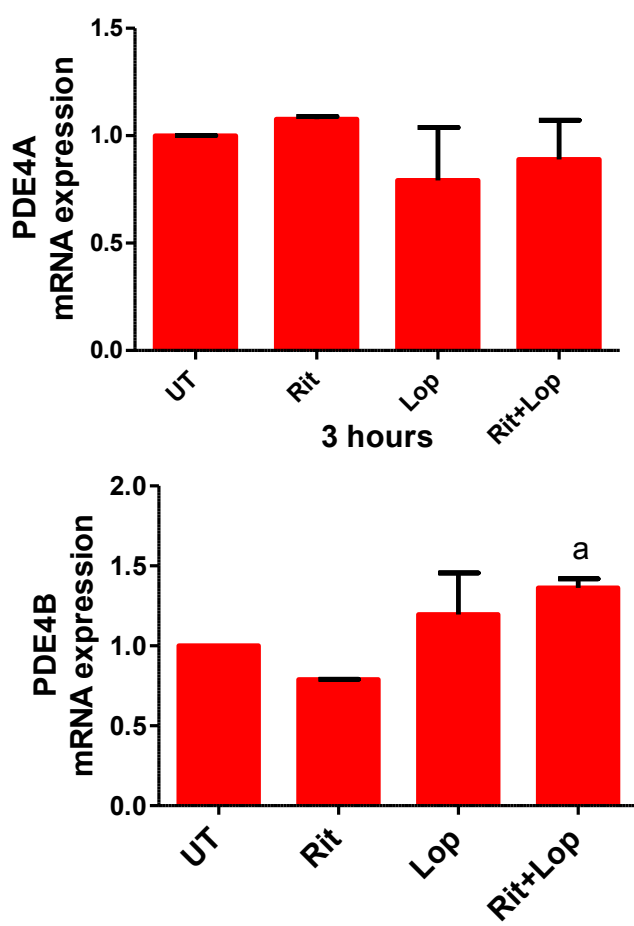

3 hours

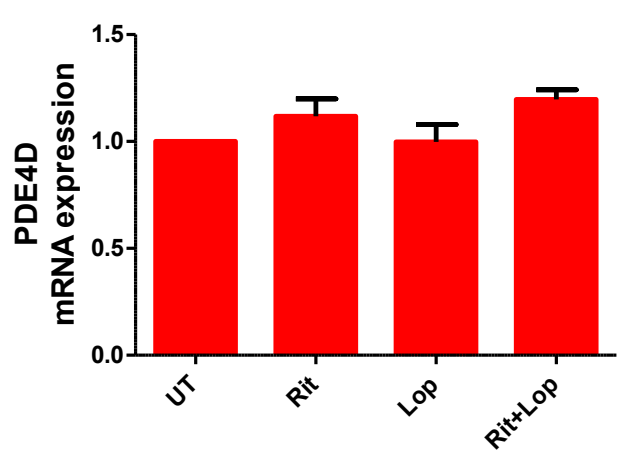

24 hours
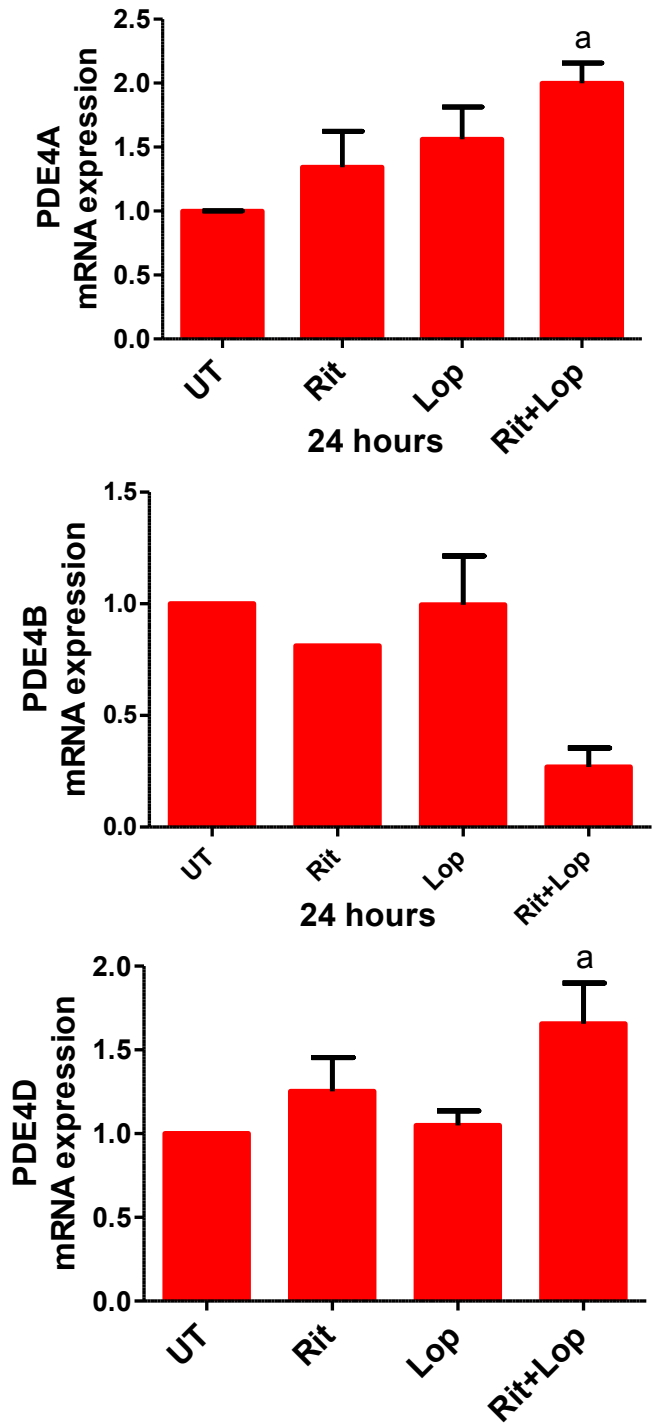

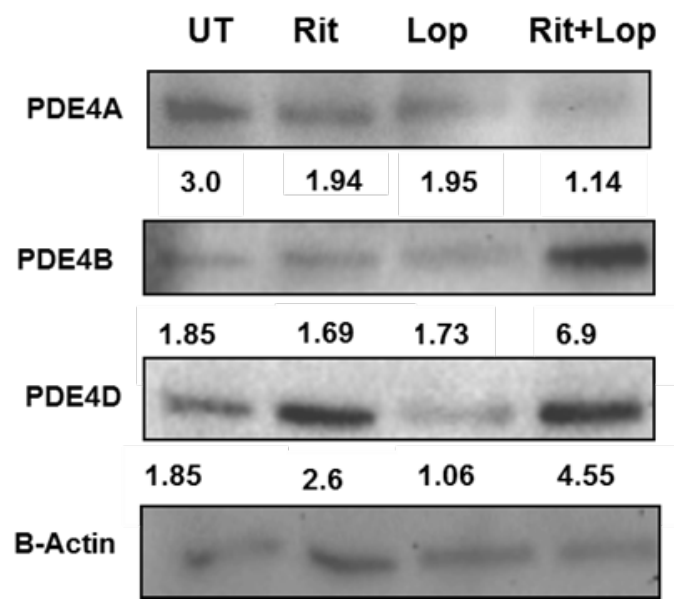


Fig. (31) HIV-PI drugs induce PDE4A, B and D mRNA and protein expression: H4IIE cells were exposed to Ritonavir (Rit) $20 \mu \mathrm{M}$ and Lopinavir (Lop)20 $\mu \mathrm{M}$ singly and in combination with or without Rolipram (Rol)10 $\mu \mathrm{M}$ at the above mentioned time points. mRNA expression was examined by real-time PCR using primers specific for PDE4A, B and D. Data represented as the mean $\pm \operatorname{SE~}(n=5)$. Statistical analysis was performed using GraphPad Prism software using oneway ANOVA followed by Bonferroni posttest. $P<0.01$. ' $a$ ' when compared to untreated (UT) 'd' when compared to Rit+Lop. Western blot analysis of PDE4A, B and $D$ was performed using total cell lysates. GAPDH was probed to ensure equivalent protein loading. Densitometry analysis was performed using ImageLab software. Density ratio was calculated using $\beta$-actin as control. Blots are representative of two independent experiments. 


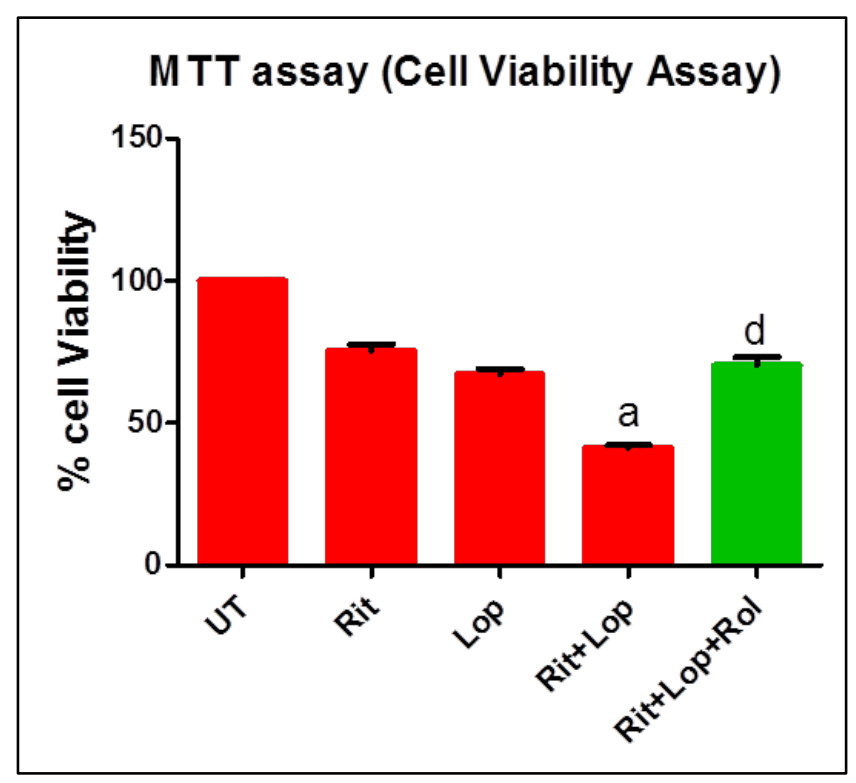

Fig.(32) HIV-PI induced hepatocyte death is partially reversed by Rolipram: H4IIE cells were exposed to Ritonavir (Rit)20 $\mu \mathrm{M}$ and Lopinavir (Lop)20 $\mu \mathrm{M}$ singly and in combination with or without Rolipram (Rol)10 $\mu \mathrm{M}$ in a 96 well format for $24 \mathrm{hrs}$. Loss of hepatocyte survival was measured by MTT assay and plotted as a bar graph with $100 \%$ survival rate in UT cells. Data represented as the mean \pm SE $(n=4)$. Statistical analysis was performed using GraphPad Prism software using one-way ANOVA followed by Bonferroni posttest. $P<0.01$. 'a' when compared to untreated (UT) 'd' when compared to Rit+Lop. 


\section{HIV-PIs induced ER-Stress was significantly attenuated by PDE4 inhibition}

Previous studies have shown that HIV-PI induces ER-stress and unfolded protein response (UPR) and apoptosis in hepatocytes [185, 210]. Specifically, the combination of HIV-PI ritonavir and lopinavir significantly activated UPR in various hepatic cells; hence, we investigated the role of phosphodiesterase in HIV-PI induced ER-stress. Studies have also shown that CHOP knockout mice were protected from HIV-PI induced ER-stress, inflammation and hepatic lipotoxicity [211]. Prolonged translation of ATF4 and 3 was shown to be responsible for CHOP transcription [212]. We investigated the effect on ATF3, 4 and $\mathrm{CHOP}$ as hallmark ER-stress proteins in HIV-PI treated cultured hepatocytes. H4IIEC3 cells were treated with Rit and Lop (20uM each) singly and in combination for a period of 6,1224 and $48 \mathrm{hrs}$. Our results showed a sustained induction of ER-stress genes ATF3, ATF4 and CHOP from 6hrs to 48hrs of PI drug exposure. Although, we did not report any significant increase of ER-stress genes by individual drugs, a trend was seen. Additionally this data was further confirmed by western blot analysis (Fig.33a,b,c,d). To address the causal role of PDE4 expression in the induction of ER-stress by PI drugs, PDE4 specific/selective inhibitor Rolipram was used. Rolipram (10uM) treatment was shown to significantly down-regulate the induction of PI-induced ER-stress markers at gene as well as protein level for all the time-points. This data indicated that PDE4 expression induced by $\mathrm{PI}$ drugs is playing regulatory role in the induction of ER-stress. 


\section{Pls induces Fas Receptor and FasL gene expression in hepatocytes}

Fas/Fas Ligand signaling is one of the most critical pathways involved in apoptosis induced cell death in hepatocytes[213]. Moreover, previous reports have shown the mechanistic link between proapoptotic ER-stress and the Fas and mitochondrial pathways of apoptosis via CamKII signaling [214]. CamKII signaling activates Fas receptor mediated apoptosis process in ER-stressed macrophages thus providing an unifying mechanistic link between UPR and apoptosis. Hence along with ER-stress expression we also investigated the role of Fas/FasL pathway in the PI-mediated apoptotic cell death in the hepatocytes. We did not report any significant up-regulation of FasL in Ritanovir and Lopinavir at $20 \mu \mathrm{M}$ treated individually. However, cells treated with the combination of Ritanovir and Lopinavir at $20 \mu \mathrm{M}$ significantly induced FasL gene expression at $24 \mathrm{hrs}$ that was further up regulated at $48 \mathrm{hrs}$. We did not get any significant induction of FasL gene expression at 6 and $12 \mathrm{hrs} \mathrm{suggesting} \mathrm{that} \mathrm{pro-apoptotic}$ FasL signaling was occurring only at later time-points (data not shown). Moreover, Western blot analysis revealed that FasL protein expression was markedly increased in the combinatorial treatment of $\mathrm{PI}$ as compared to untreated in the hepatocytes. Similarly, Fas R was also significantly up-regulated by PI combinatorial treatment on the hepatocytes. Rolipram treatment significantly reversed this PI-drug induced up-regulation. This data showed for the first time that cAMP depletion/PDE4 inhibition could have an influence on Fas/FasL mediated drug-induced cell death in the hepatocytes (Fig 34). 


\section{PDE4 inhibition down-regulates FasL expression in the hepatocytes}

Previously, we have shown that PIs modulate PDE4/cAMP levels in the hepatocytes. Hence we wanted to investigate the role of PDE4/cAMP metabolism in regulating FasL gene expression. Rat hepatocytes were treated with Rit/Lop and rolipram. As expected, FasL gene expression as well as protein was completely abrogated by Rolipram (Fig.34). This data indicates that FasL plays a major role in PI induced cell death in hepatocytes and more importantly, PDE4 inhibition via rolipram significantly protected hepatocytes by downregulating FasL-induced cell death.

\section{Role of cAMP-PKA signaling in PI induced ER-stress and FasL mediated cell death in hepatocytes}

Previously, we have shown that ER-Stress as well as FasL-mediated cell death is regulated by PDE4 expression. In an assumption that the PI drugs compromised cellular cAMP levels we investigated the role of cAMP signaling in $\mathrm{Pl}$-induced upregulation of ER-stress and FasL expression. cAMP exerts its downstream effects by phosphorylating and activating its targets such as protein kinase $A(P K A)$ and Exchange proteins activated by cAMP (EPAC). Hence to determine whether cAMP-PKA signaling is involved in PI-induced ER-stress in hepatocyte we examined the effects of a PKA inhibitor $(\mathrm{H}-89)(5 \mathrm{uM})$ along with HIV-PI and rolipram. H4IIEC3 cells were treated with Rit +Lop with/without rolipram. As shown previously, rolipram treatment significantly down-regulated ER-stress genes ATF3, 4 and $\mathrm{CHOP}$ as well as FasL at 24hrs. Addition of $\mathrm{H}-89$ to rolipram and PI-treated cells significantly reversed the rolipram effect of ERstress and FasL suppression (Fig. 35). This data suggests that cAMP-PKA could 
play a key role in regulating ER-stress and FasL mediated cell death in hepatocytes. We also speculate that PI drugs could be affecting PKA phosphorylation leading to its inactivation in the hepatocytes and thus affecting cAMP/PKA signaling. 
6 hours

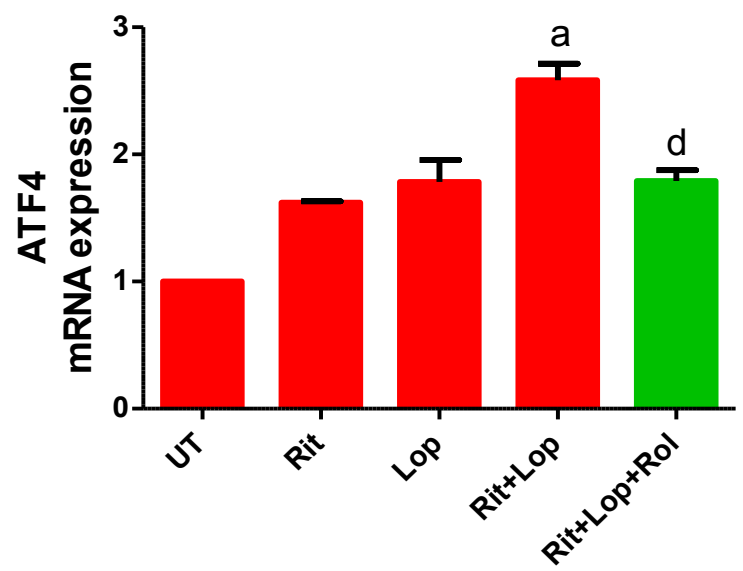

24 hours

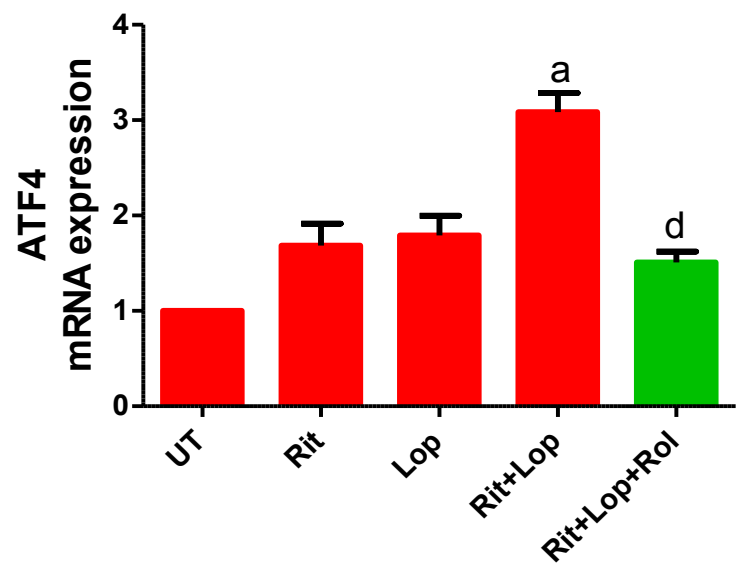

12 hours

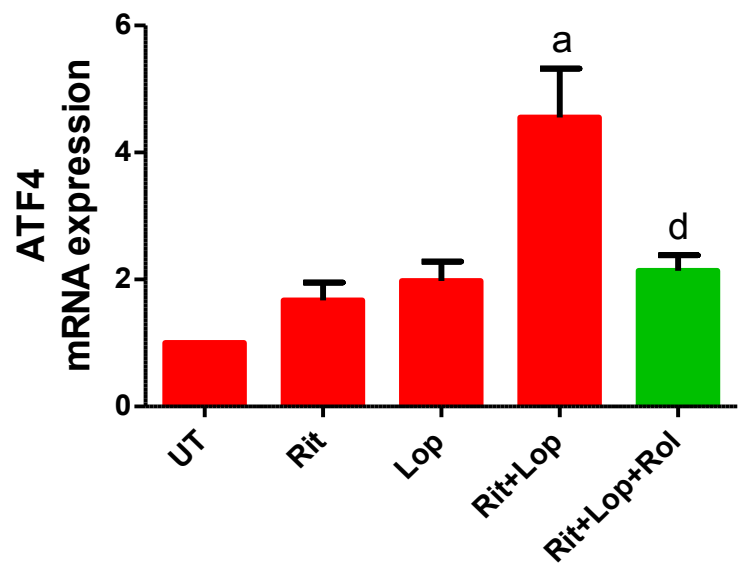

48 hours

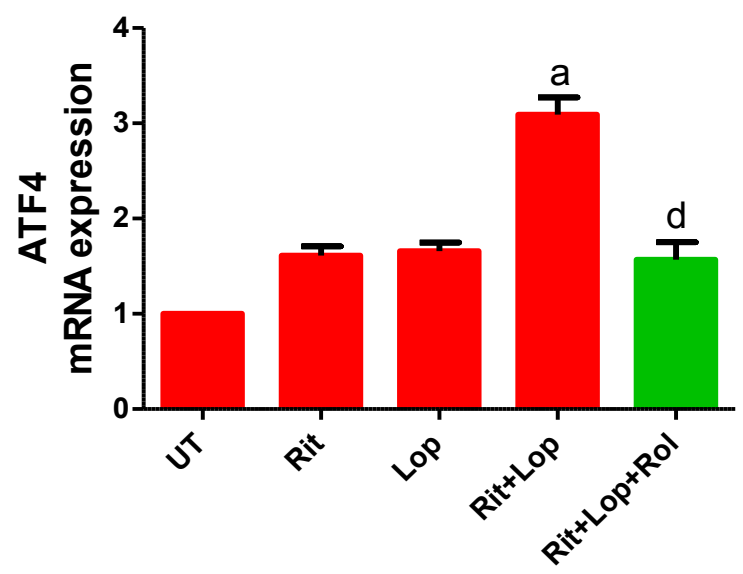

Fig. (33a) HIV-PI mediated ER-stress gene, ATF4 expression is down-regulated by PDE4 inhibitor, Rolipram: H4IIE cells were exposed to Ritonavir (Rit) $20 \mu \mathrm{M}$ and Lopinavir (Lop) $20 \mu \mathrm{M}$ singly and in combination with or without Rolipram (Rol) $10 \mu \mathrm{M}$ at the above mentioned time points. mRNA expression was examined by real-time PCR using primers specific for ATF4. Data represented as the mean $\pm S E(n=5)$. Statistical analysis was performed using GraphPad Prism software using one-way ANOVA followed by Bonferroni posttest. $P<0.01$. ' $a$ ' when compared to untreated (UT) 'd' when compared to Rit+Lop. 
6 hours

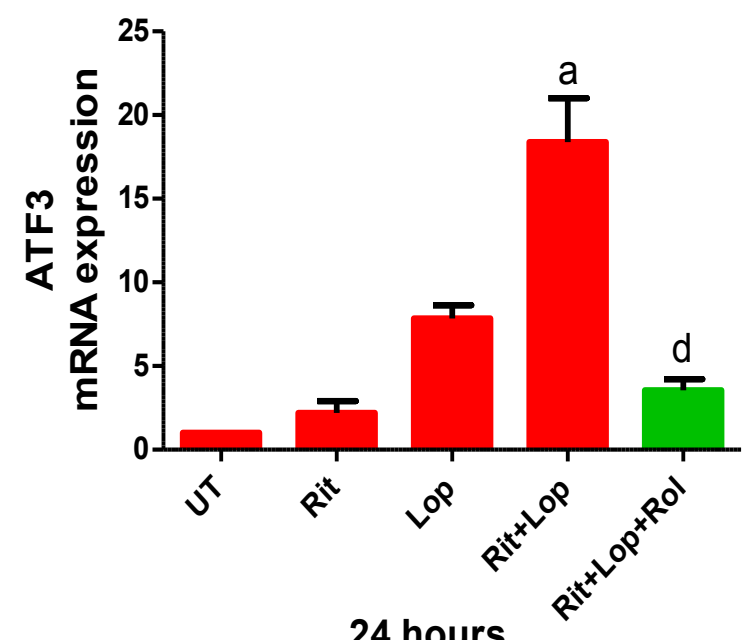

24 hours

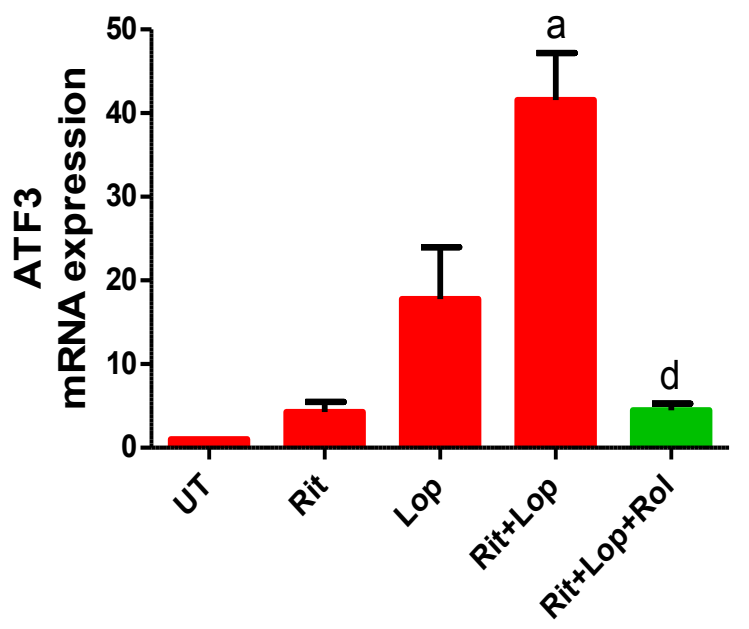

12 hours

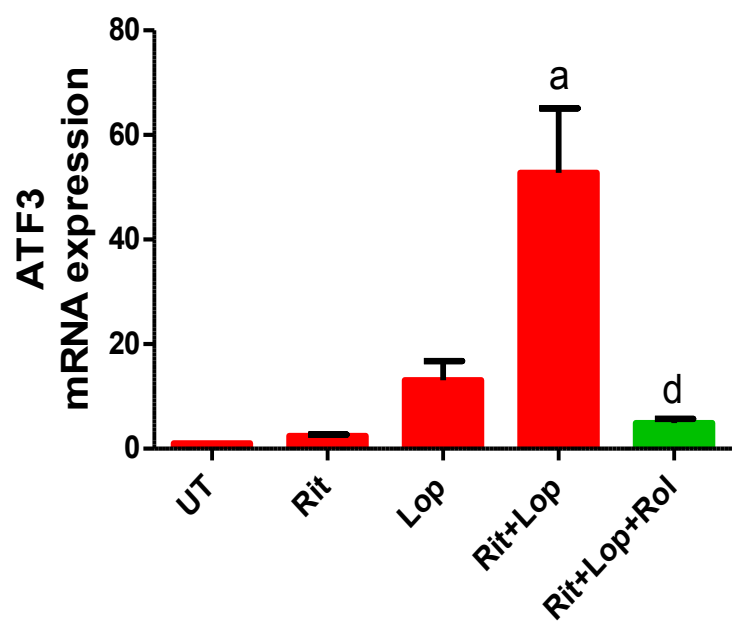

48 hours

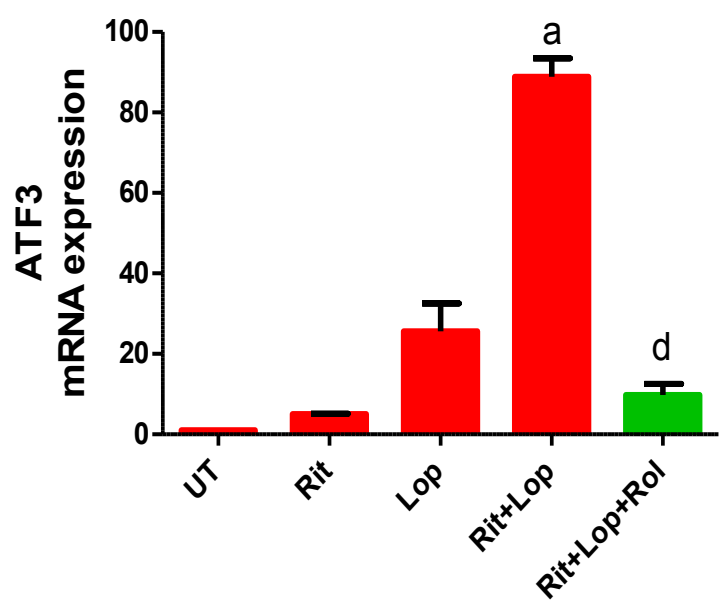

Fig. (33b) HIV-PI mediated ER-stress gene ATF3 expression is down-regulated by PDE4 inhibitor, Rolipram: H4IIE cells were exposed to Ritonavir (Rit)20 $\mu \mathrm{M}$ and Lopinavir (Lop)20 $\mu \mathrm{M}$ singly and in combination with or without Rolipram (Rol) $10 \mu \mathrm{M}$ at the above mentioned time points. mRNA expression was examined by real-time PCR using primers specific for ATF3. Data represented as the mean $\pm S E(n=5)$. Statistical analysis was performed using GraphPad Prism software using one-way ANOVA followed by Bonferroni posttest. $P<0.01$. ' $a$ ' when compared to untreated (UT) 'd' when compared to Rit+Lop. 
6 hours

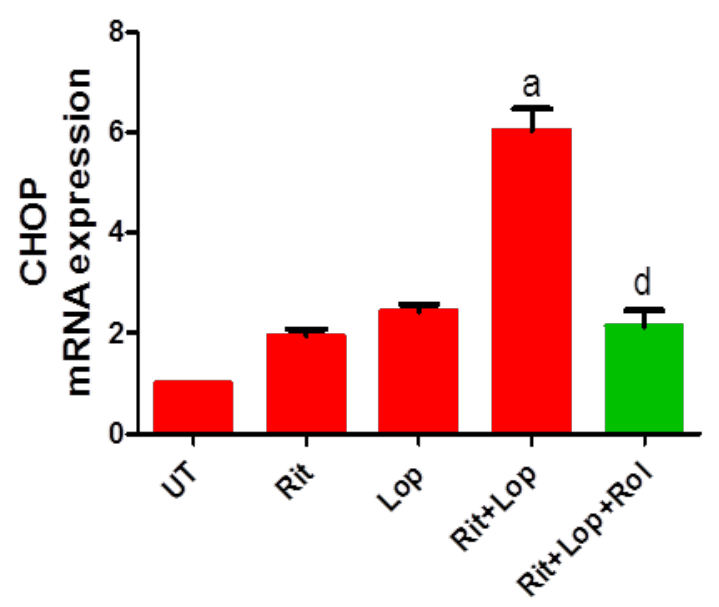

24 hours

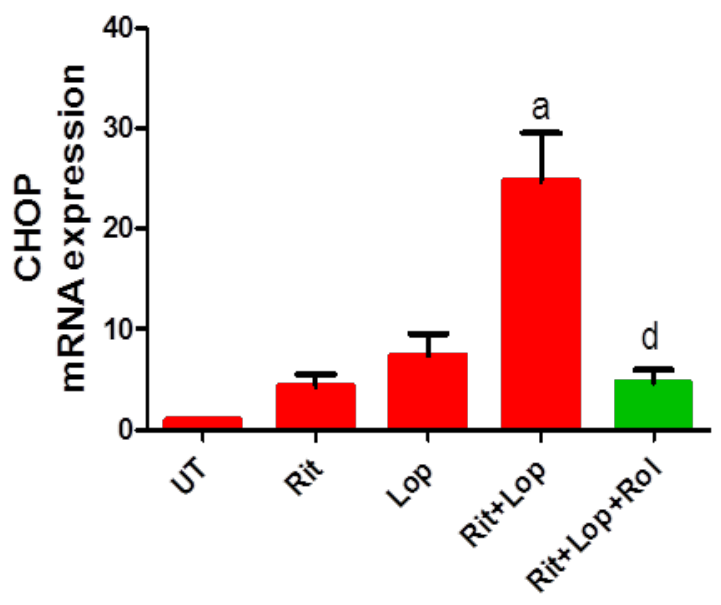

12 hours

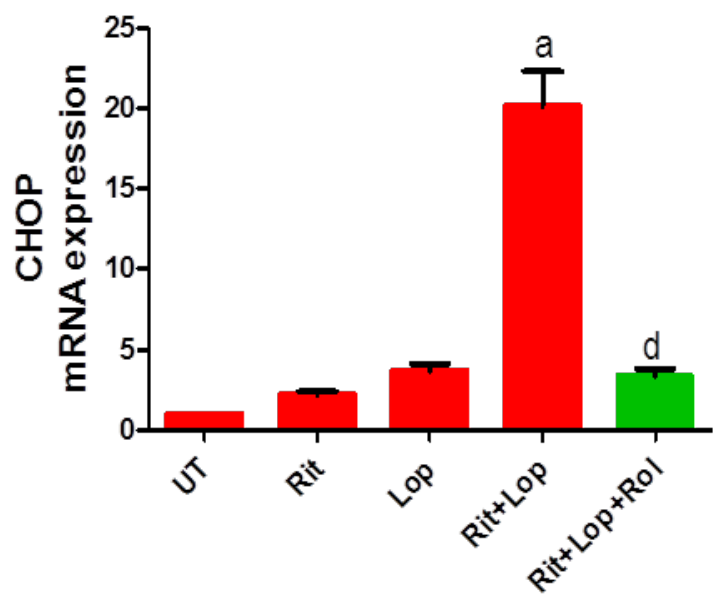

48 hours

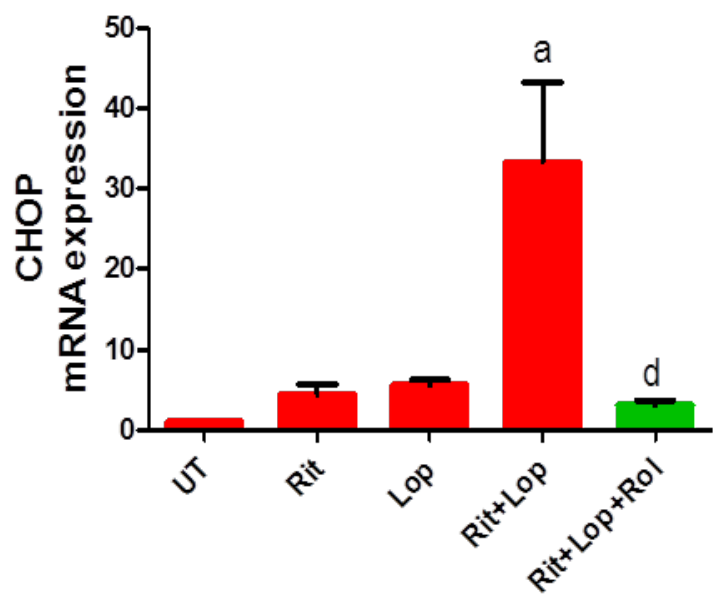

Fig. (33c) HIV-PI mediated ER-stress gene CHOP expression is down-regulated by PDE4 inhibitor, Rolipram: H4IIE cells were exposed to Ritonavir (Rit)20 $\mu \mathrm{M}$ and Lopinavir (Lop)20 $\mu \mathrm{M}$ singly and in combination with or without Rolipram (Rol)10 $\mu \mathrm{M}$ at the above mentioned time points. mRNA expression was examined by real-time PCR using primers specific for CHOP. Data represented as the mean \pm SE $(n=5)$. Statistical analysis was performed using GraphPad Prism software using one-way ANOVA followed by Bonferroni posttest. $P<0.01$. 'a' when compared to untreated (UT) 'd' when compared to Rit+Lop. 


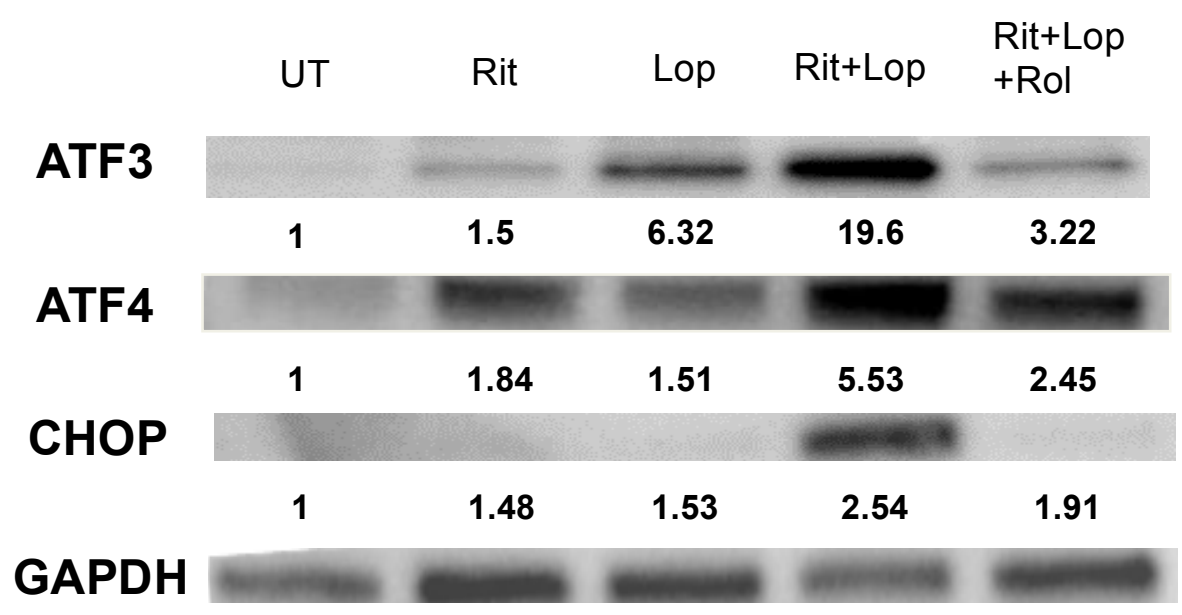

Fig. (33d) HIV-PI mediated ER-stress protein expression is significantly attenuated by PDE4 inhibitor, Rolipram: H4IIE cells were exposed to Ritonavir (Rit)20 $\mu \mathrm{M}$ and Lopinavir (Lop)20 $\mu \mathrm{M}$ singly and in combination with or without Rolipram (Rol) $10 \mu \mathrm{M}$ at $24 \mathrm{hrs}$. Western blot analysis of ATF3, ATF4 and CHOP was performed using total cell lysates. GAPDH was probed to ensure equivalent protein loading. Densitometry analysis was performed using ImageLab software. Density ratio was calculated using GAPDH as control. Blots are representative of two independent experiments. 
24 hours

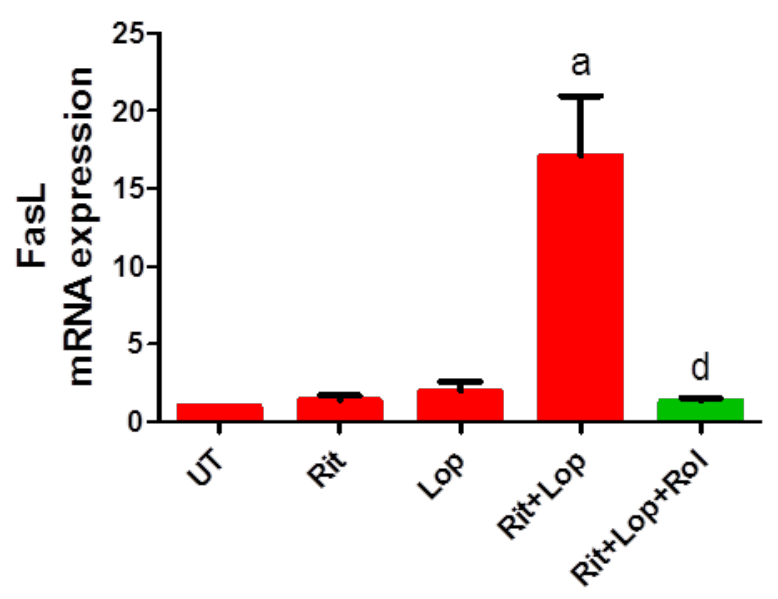

FasL

B-actin

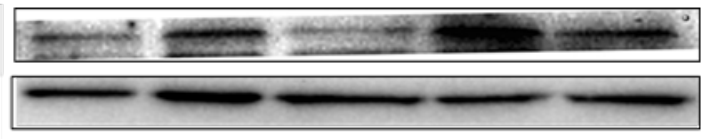

UT
Rit
Rit+Lop Rit+Lop + Rol

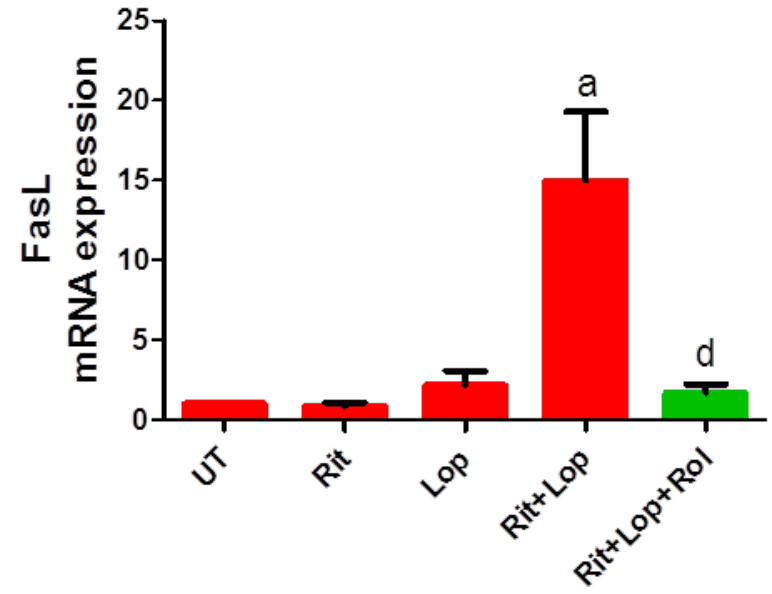

24 hours

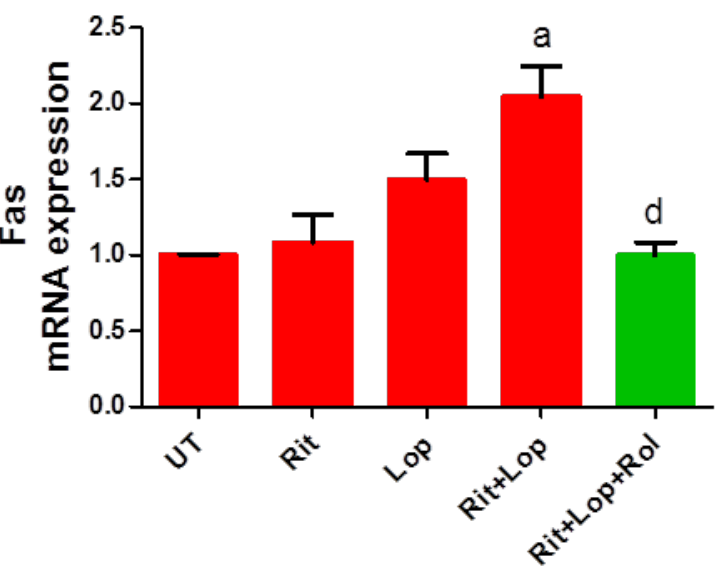

Fig (34) HIV-PI mediated Fas and FasL expression is down-regulated by PDE4 inhibitor, Rolipram: H4IIE cells were exposed to Ritonavir (Rit)20 $\mu \mathrm{M}$ and Lopinavir (Lop)20 $\mu \mathrm{M}$ singly and in combination with or without Rolipram (Rol) $10 \mu \mathrm{M}$ at the above mentioned time points. mRNA expression was examined by real-time PCR using primers specific for FasL and Fas. Data represented as the mean $\pm S E(n=5)$. Statistical analysis was performed using GraphPad Prism software using one-way ANOVA followed by Bonferroni posttest. $P<0.01$. ' $a$ ' when compared to untreated (UT) 'd' when compared to Rit+Lop. Western blot analysis of FasL was performed using total cell lysates. $\beta$-actin was probed to ensure equivalent protein loading. Densitometry analysis was performed using ImageLab software. Density ratio was calculated using GAPDH as control. Blots are representative of two independent experiments. 

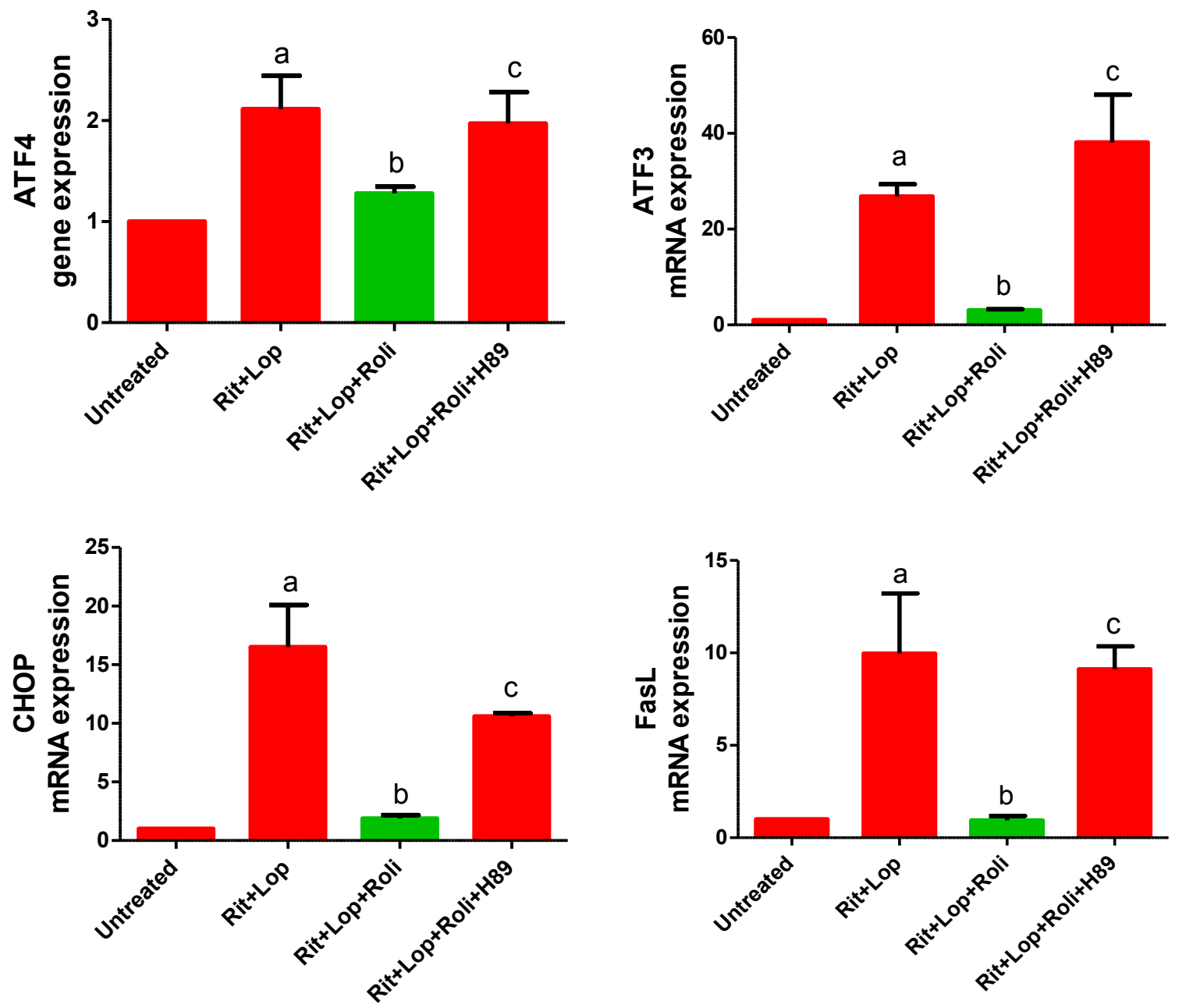

Fig (35) PKA inhibitor $\mathrm{H}-89$ significantly reversed the protective effect of Rolipram on ER-stress and FasL gene expression: H4IIE cells were exposed to Ritonavir (Rit)20 $\mu \mathrm{M}$ and Lopinavir (Lop)20 $\mu \mathrm{M}$ singly and in combination with or without Rolipram (Rol) $10 \mu \mathrm{M}$ along with PKA inhibitor $\mathrm{H}-89(5 \mu \mathrm{M})$ at 24hrs. mRNA expression was examined by real-time PCR using primers specific for ER stress gene ATF4,3, CHOP and FasL. Data represented as the mean \pm SE $(n=5)$. Statistical analysis was performed using GraphPad Prism software using oneway ANOVA followed by Bonferroni posttest. $P<0.01$. ' $a$ ' when compared to untreated (UT) 'b' when compared to Rit+Lop and 'c' when compared to Rit+Lop+Rol. 


\section{DISCUSSION}

HIV-protease inhibitors are used as combinatorial therapy and have significant therapeutic potential in the treatment of HIV-infection [215]. However, the prolong use of Pls has significant hepatotoxic effects [183]. Although, PIinduced hepatotoxicity has been examined, the underlying mechanisms are not yet fully elucidated. Previous studies have shown that PI-induced endoplasmic reticulum (ER) stress response play an important role in the development of inflammation, dyslipidemia, and insulin resistance [185, 216-219]. In the present work, molecular mechanisms driving PI-induced ER stress were investigated. Specifically, since combination of Rit and Lop constitutes a common PI-treatment regimen for HIV patients, their hepatotoxic effects were examined. The dose of Rit $(20 \mu \mathrm{M})$ and Lop $(20 \mu \mathrm{M})$ employed in the present studies was based on earlier clinical and experimental findings of anti-viral efficacy and hepatotoxicity $[184,185,220-222]$. As seen in earlier studies, our initial examination of the effect of Rit and Lop on hepatocytes showed that their combination was able to significantly induce ER stress. Particularly, expression of critical ER-Stress proteins such as ATF3, 4 and a known pro-apoptotic protein GADD153/CHOP were significantly up-regulated. To address the mechanisms underlying PIinducible ER stress we investigated the potential role of PDE4 expression that leads to a decline in cellular cAMP levels. The rationale for examining PDE4 expression was provided by the earlier studies done by our research group [203]. 
These studies have demonstrated the major pathogenic role of PDE4 expression and cAMP metabolism in experimental alcoholic liver disease and cholestatic liver inflammation, injury and fibrosis [203, 209, 223]. Additionally, work done by others has shown that increased cellular cAMP levels can effectively block hepatocyte apoptotic death induced by various stimuli including bile-acids, Fas and TNF $[209,223,224]$. Our data showed that Phosphodiesterase 4 (PDE4) plays a pathogenic role in PI-induced ER stress and apoptotic cell death in hepatocytes. Treatment of hepatocytes with the combination of Rit and Lop (Rit+Lop) differentially induced the expression of PDE4 subfamily members. Specifically, PDE4B mRNA expression was observed to be induced as early as $3 \mathrm{hrs}$ post-treatment, whereas, there was a significantly delayed induction of PDE4A and D mRNA expression occurring at $24 \mathrm{~h}$. Interestingly, examination of protein expression revealed only PDE4B and D subfamily members at $24 \mathrm{~h}$. Since PDE4 members are the major cAMP-specific phosphodiesterase, their rapid and sustained induction by PIs potentially represents a pathogenic event decreasing hepatocyte cAMP levels and downstream signaling. Since we observed an increase in the expression of both PDE4s and ER stress proteins in Rit+Lop treated hepatocytes, it is possible that PDE4 upregulation and ER-Stress are causally linked. The causal role of PDE4 was investigated by pre-treating the hepatocytes with Rolipram - a PDE4 specific inhibitor. Notably, PDE4 inhibition was able to abrogate Rit+Lop induced expression of ER stress genes and significantly enhanced hepatocyte survival. These data identify for the first time, PDE4 expression as the key mechanism that underlies PI-inducible ER stress 
and hepatotoxicity. With regards to ER stress mediated cell death, the induction of pro-apoptotic GADD153/CHOP is well correlated with the onset of ER stressassociated apoptosis and has been observed to be induced by Rit+Lop in hepatocytes [225-227]. However, besides induction of GADD153/CHOP, ER stress has also been linked to Fas and mitochondrial apoptosis pathways [214]. Importantly, Fas-FasL mediated hepatocyte death has been demonstrated to contribute to drug induced hepatocyte cytotoxicity [228, 229]. Also, in relevance to this study, it has been demonstrated that cAMP inhibits FasL expression and apoptotic cell death in lymphocytes [230]. Since we identified that Rit+Lop significantly induces CAMP degrading PDE4 expression that is causally linked with the development of ER stress, we further examined the effect on Fas and FasL expression. The data obtained showed for the first time that Rit+Lop can significantly induce the expression of both Fas and FasL that are known to induce apoptosis and hepatocyte death. Notably, PDE4 expression was also determined to play a causal role in the Rit+Lop-inducible Fas and FasL expression. Inhibition of PDE4 leads to increased cellular levels of cAMP and a major component of the cellular effects of cAMP are mediated by cAMP dependent PKA (cAMP-PKA) activation and signaling [231]. Accordingly, our data suggested that the inhibitory effect of PDE4 inhibition decreasing ER stress, Fas and FasL expression could be mediated via cAMP-PKA activation. This aspect was evaluated by examining the effect of cAMP-PKA inhibitor on hepatocytes that were treated with Rit+Lop and Rolipram. The data obtained strongly suggests that CAMP-PKA activity is essential for the prevention of ER 
stress, Fas and FasL expression and hepatotoxicity induced by combinatorial PI (Rit+Lop) treatment. Taken together, these data demonstrate that PDE4 expression and consequent decrease in cAMP-PKA activation/signaling, plays a key pathogenic role in the induction of Rit+Lop hepatotoxicity by both individually or coordinately activating ER stress and Fas/FasL expression. Significantly, these data also suggest that targeting PDE4 activity has therapeutic potential in the treatment of Pl-induced hepatotoxicity in HIV-infected patients. 


\section{SUMMARY AND CONCLUSIONS}

In the present study we investigated the role of Phosphodiesterase 4 (PDE4) and ER-Stress as significant pathogenic mechanism in Highly Active Anti-retroviral Therapy (HAART) Induced Hepatotoxicity. The main purpose of HAART is to significantly improve the quality of life by suppressing the viral load and its replication in the body of an HIV (Human Immunodeficiency Virus) infected individual. This is achieved by administering multiple drug therapy used in HAART. Protease Inhibitors (PIs) are being used as one of the therapies in the initial treatment of HIV infection. Even though these drugs have a positive impact on the patients, they are associated with several undesirable consequences in their long-term treatment. Some of the major complications of HAART are hepatotoxicity and lipodystrophy leading to its discontinuation. Hence it becomes highly relevant to investigate the mechanisms involved in PI induced hepatotoxicity. Recent studies have shown the involvement of ER stress and subsequent activation of the unfolded protein response (UPR) resulting in apoptosis/hepatocyte death as one of the cellular mechanisms of HIV-PI induced metabolic disorders.

Additionally, cAMP is a known anti-apoptotic or a pro-survival signal in many different cell types including hepatocytes. Specifically in hepatocytes, cAMP is involved in the protective mechanism from various insults such as Fas Ligand, TNF- $\alpha$, and hydrophobic bile acids. Our recent work shows that 
PDE4/CAMP metabolism plays a significant role in alcohol-induced hepatic steatosis and injury. Phosphodiesterase (PDEs), a family of enzymes precisely regulates intracellular levels of cAMP via adenylyl cyclase are involved in their synthesis as well as degradation. Our recent work shows that PDE4/cAMP metabolism plays a significant role in alcohol-induced hepatic steatosis and injury. Hence in the present study, we examined the mechanisms underlying HIV-PI induced hepatic ER stress and toxicity with a particular emphasis on PDE4 family of enzymes.

The effects of clinically used HIV-PIs (Ritonavir and Lopinavir) were examined both in a rat hepatoma cell line as well as rat primary hepatocytes. These studies demonstrated that Pls led to a significant loss of hepatocyte survival. Notably, inhibition of PDE4 by a specific PDE4-inhibitor, rolipram, markedly attenuated hepatotoxicity induced by PIs. Mechanistic role of PDE4 showed that PDE4 inhibition significantly decreases the expression of the ER stress related proteins C/EBP homologous protein (CHOP), Activating transcription factor (ATF-4 and -3) induced by Pls. Furthermore, examination of the PDE4/cAMP-regulated downstream signaling demonstrated the involvement of PKA. Specifically, cAMP-PKA inhibitor H-89 reversed the rolipram effect of down-regulating the expression of the Pl-induced ER-stress genes. These data identify an important pathogenic/mechanistic role for PDE4 regulated cAMP-PKA in the development of HIV-PI induced ER-stress leading to hepatic steatosis and injury. 
SUMMARY AND CONCLUSIONS
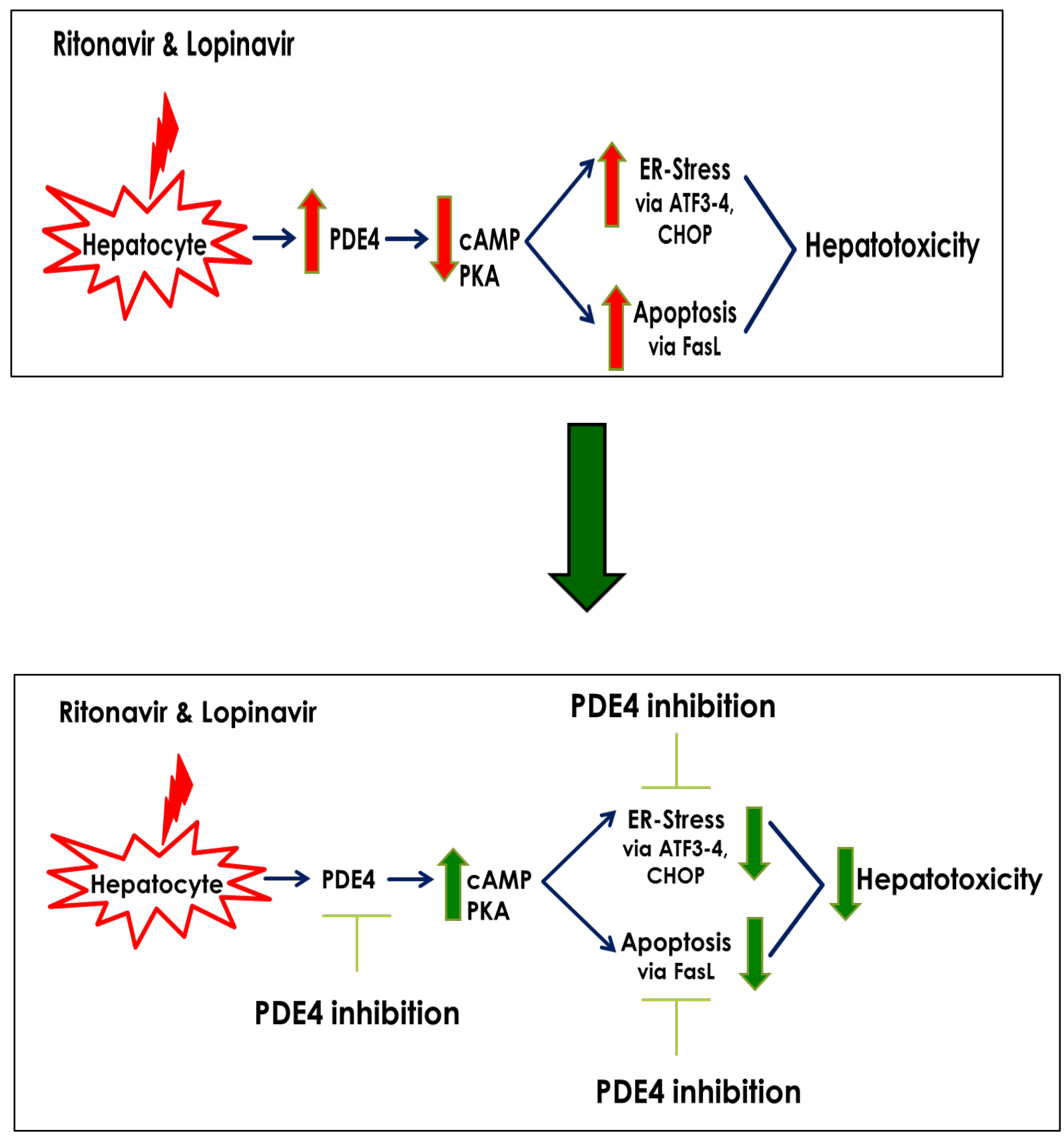


\section{REFERENCES}

1. Mathurin, P., et al., Fibrosis progression occurs in a subgroup of heavy drinkers with typical histological features. Aliment Pharmacol Ther, 2007. 25(9): p. 1047-54.

2. Lefkowitch, J.H., Morphology of alcoholic liver disease. Clin Liver Dis, 2005. 9(1): p. 3753.

3. O'Shea, R.S., et al., Alcoholic liver disease. Hepatology, 2010. 51(1): p. 307-28.

4. Tsukamoto, H., et al., "Second hit" models of alcoholic liver disease. Semin Liver Dis, 2009. 29(2): p. 178-87.

5. Wilfred de Alwis, N.M. and C.P. Day, Genetics of alcoholic liver disease and nonalcoholic fatty liver disease. Semin Liver Dis, 2007. 27(1): p. 44-54.

6. Postic, C. and J. Girard, Contribution of de novo fatty acid synthesis to hepatic steatosis and insulin resistance: lessons from genetically engineered mice. J Clin Invest, 2008.

118(3): p. 829-38.

7. Browning, J.D. and J.D. Horton, Molecular mediators of hepatic steatosis and liver injury. J Clin Invest, 2004. 114(2): p. 147-52.

8. Reddy, J.K. and M.S. Rao, Lipid metabolism and liver inflammation. II. Fatty liver disease and fatty acid oxidation. Am J Physiol Gastrointest Liver Physiol, 2006. 290(5): p. G852-8.

9. McGarry, J.D., et al., Regulation of ketogenesis and the renaissance of carnitine palmitoyltransferase. Diabetes Metab Rev, 1989. 5(3): p. 271-84.

10. McGarry, J.D. and D.W. Foster, Regulation of hepatic fatty acid oxidation and ketone body production. Annu Rev Biochem, 1980. 49: p. 395-420.

11. Esser, V., et al., Inhibitors of mitochondrial carnitine palmitoy/transferase I limit the action of proteases on the enzyme. Isolation and partial amino acid analysis of a truncated form of the rat liver isozyme. J Biol Chem, 1993. 268(8): p. 5810-6.

12. Esser, V., et al., Expression of a CDNA isolated from rat brown adipose tissue and heart identifies the product as the muscle isoform of carnitine palmitoyltransferase I (M-CPT I). $M-C P T I$ is the predominant CPT I isoform expressed in both white (epididymal) and brown adipocytes. J Biol Chem, 1996. 271(12): p. 6972-7.

13. Yamazaki, N., et al., High expression of a novel carnitine palmitoy/transferase I like protein in rat brown adipose tissue and heart: isolation and characterization of its CDNA clone. FEBS Lett, 1995. 363(1-2): p. 41-5.

14. Serviddio, G., et al., Oxidation of hepatic carnitine palmitoyl transferase-I (CPT-I) impairs fatty acid beta-oxidation in rats fed a methionine-choline deficient diet. PLoS One, 2011. 6(9): p. e24084.

15. Stefanovic-Racic, M., et al., A moderate increase in carnitine palmitoyltransferase $1 a$ activity is sufficient to substantially reduce hepatic triglyceride levels. Am J Physiol Endocrinol Metab, 2008. 294(5): p. E969-77.

16. Kim, M.J., et al., Dietary umbelliferone attenuates alcohol-induced fatty liver via regulation of PPARalpha and SREBP-1c in rats. Alcohol, 2014. 48(7): p. 707-15.

17. Kirpich, I., et al., Binge ethanol-induced HDAC3 down-regulates Cpt1alpha expression leading to hepatic steatosis and injury. Alcohol Clin Exp Res, 2013. 37(11): p. 1920-9.

18. Ajmo, J.M., et al., Resveratrol alleviates alcoholic fatty liver in mice. Am J Physiol Gastrointest Liver Physiol, 2008. 295(4): p. G833-42. 
19. Kang, X., et al., Zinc supplementation reverses alcohol-induced steatosis in mice through reactivating hepatocyte nuclear factor-4alpha and peroxisome proliferator-activated receptor-alpha. Hepatology, 2009. 50(4): p. 1241-50.

20. Lazarow, P.B. and Y. Fujiki, Biogenesis of peroxisomes. Annu Rev Cell Biol, 1985. 1: p. 489-530.

21. Wanders, R.J. and J.M. Tager, Lipid metabolism in peroxisomes in relation to human disease. Mol Aspects Med, 1998. 19(2): p. 69-154.

22. Wanders, R.J. and H.R. Waterham, Biochemistry of mammalian peroxisomes revisited. Annu Rev Biochem, 2006. 75: p. 295-332.

23. Delille, H.K., et al., Pex11pbeta-mediated growth and division of mammalian peroxisomes follows a maturation pathway. J Cell Sci, 2010. 123(Pt 16): p. 2750-62.

24. Fagarasanu, A., M. Fagarasanu, and R.A. Rachubinski, Maintaining peroxisome populations: a story of division and inheritance. Annu Rev Cell Dev Biol, 2007. 23: p. 32144.

25. Koch, J., et al., PEX11 family members are membrane elongation factors that coordinate peroxisome proliferation and maintenance. J Cell Sci, 2010. 123(Pt 19): p. 3389-400.

26. Weng, H., et al., Pex11alpha deficiency impairs peroxisome elongation and division and contributes to nonalcoholic fatty liver in mice. Am J Physiol Endocrinol Metab, 2013. 304(2): p. E187-96.

27. Braissant, O., et al., Differential expression of peroxisome proliferator-activated receptors (PPARs): tissue distribution of PPAR-alpha, -beta, and-gamma in the adult rat. Endocrinology, 1996. 137(1): p. 354-66.

28. Napal, L., P.F. Marrero, and D. Haro, An intronic peroxisome proliferator-activated receptor-binding sequence mediates fatty acid induction of the human carnitine palmitoyltransferase 1A. J Mol Biol, 2005. 354(4): p. 751-9.

29. Ip, E., et al., Central role of PPARalpha-dependent hepatic lipid turnover in dietary steatohepatitis in mice. Hepatology, 2003. 38(1): p. 123-32.

30. Yu, S., S. Rao, and J.K. Reddy, Peroxisome proliferator-activated receptors, fatty acid oxidation, steatohepatitis and hepatocarcinogenesis. Curr Mol Med, 2003. 3(6): p. 56172.

31. Aoyama, T., et al., Altered constitutive expression of fatty acid-metabolizing enzymes in mice lacking the peroxisome proliferator-activated receptor alpha (PPARalpha). J Biol Chem, 1998. 273(10): p. 5678-84.

32. Hardwick, J.P., Cytochrome P450 omega hydroxylase (CYP4) function in fatty acid metabolism and metabolic diseases. Biochem Pharmacol, 2008. 75(12): p. 2263-75.

33. Knight, B.L., et al., A role for PPARalpha in the control of SREBP activity and lipid synthesis in the liver. Biochem J, 2005. 389(Pt 2): p. 413-21.

34. Song, S., et al., Peroxisome proliferator activated receptor alpha (PPARalpha) and PPAR gamma coactivator (PGC-1alpha) induce carnitine palmitoyltransferase IA (CPT-1A) via independent gene elements. Mol Cell Endocrinol, 2010. 325(1-2): p. 54-63.

35. Vega, R.B., J.M. Huss, and D.P. Kelly, The coactivator PGC-1 cooperates with peroxisome proliferator-activated receptor alpha in transcriptional control of nuclear genes encoding mitochondrial fatty acid oxidation enzymes. Mol Cell Biol, 2000. 20(5): p. 1868-76.

36. Galli, A., et al., The transcriptional and DNA binding activity of peroxisome proliferatoractivated receptor alpha is inhibited by ethanol metabolism. A novel mechanism for the development of ethanol-induced fatty liver. J Biol Chem, 2001. 276(1): p. 68-75. 
37. Fischer, M., et al., Peroxisome proliferator-activated receptor alpha (PPARalpha) agonist treatment reverses PPARalpha dysfunction and abnormalities in hepatic lipid metabolism in ethanol-fed mice. J Biol Chem, 2003. 278(30): p. 27997-8004.

38. Nanji, A.A., et al., Alcoholic liver injury in the rat is associated with reduced expression of peroxisome proliferator-alpha (PPARalpha)-regulated genes and is ameliorated by PPARalpha activation. J Pharmacol Exp Ther, 2004. 310(1): p. 417-24.

39. Hayhurst, G.P., et al., Hepatocyte nuclear factor 4 alpha (nuclear receptor $2 \mathrm{A1}$ ) is essential for maintenance of hepatic gene expression and lipid homeostasis. Mol Cell Biol, 2001. 21(4): p. 1393-403.

40. Inoue, Y., et al., Hepatocyte nuclear factor 4 alpha is a central regulator of bile acid conjugation. J Biol Chem, 2004. 279(4): p. 2480-9.

41. Martinez-Jimenez, C.P., et al., Hepatocyte nuclear factor 4alpha coordinates a transcription factor network regulating hepatic fatty acid metabolism. Mol Cell Biol, 2010. 30(3): p. 565-77.

42. Louet, J.F., et al., The coactivator PGC-1 is involved in the regulation of the liver carnitine palmitoyltransferase I gene expression by CAMP in combination with HNF4 alpha and CAMP-response element-binding protein (CREB). J Biol Chem, 2002. 277(41): p. 379918000.

43. Steffen, M.L., et al., Expression of the rat liver carnitine palmitoy/transferase I (CPTlalpha) gene is regulated by Sp1 and nuclear factor Y: chromosomal localization and promoter characterization. Biochem J, 1999. 340 ( Pt 2): p. 425-32.

44. Wei, T., et al., Flavonoid ingredients of Ginkgo biloba leaf extract regulate lipid metabolism through Sp1-mediated carnitine palmitoyltranferase $1 \mathrm{~A}$ up-regulation. J Biomed Sci, 2014. 21: p. 87.

45. Sadana, P. and E.A. Park, Characterization of the transactivation domain in the peroxisome-proliferator-activated receptor gamma co-activator (PGC-1). Biochem J, 2007. 403(3): p. 511-8.

46. Rodgers, J.T., et al., Nutrient control of glucose homeostasis through a complex of PGC1alpha and SIRT1. Nature, 2005. 434(7029): p. 113-8.

47. Gerhart-Hines, Z., et al., Metabolic control of muscle mitochondrial function and fatty acid oxidation through SIRT1/PGC-1alpha. EMBO J, 2007. 26(7): p. 1913-23.

48. Puigserver, P., et al., Activation of PPARgamma coactivator-1 through transcription factor docking. Science, 1999. 286(5443): p. 1368-71.

49. Lieber, C.S., et al., Effect of chronic alcohol consumption on Hepatic SIRT1 and PGC1alpha in rats. Biochem Biophys Res Commun, 2008. 370(1): p. 44-8.

50. Alenghat, T., et al., Nuclear receptor corepressor and histone deacetylase 3 govern circadian metabolic physiology. Nature, 2008. 456(7224): p. 997-1000.

51. Horton, J.D., J.L. Goldstein, and M.S. Brown, SREBPs: activators of the complete program of cholesterol and fatty acid synthesis in the liver. J Clin Invest, 2002. 109(9): p. 1125-31.

52. $\mathrm{Ji}, \mathrm{C}$. and N. Kaplowitz, Betaine decreases hyperhomocysteinemia, endoplasmic reticulum stress, and liver injury in alcohol-fed mice. Gastroenterology, 2003. 124(5): p. 1488-99.

53. Schug, T.T. and X. Li, Sirtuin 1 in lipid metabolism and obesity. Ann Med, 2011. 43(3): p. 198-211.

54. Chang, H.C. and L. Guarente, SIRT1 and other sirtuins in metabolism. Trends Endocrinol Metab, 2014. 25(3): p. 138-45.

55. Revollo, J.R. and X. Li, The ways and means that fine tune Sirt1 activity. Trends Biochem Sci, 2013. 38(3): p. 160-7. 
56. You, M., et al., Mammalian sirtuin 1 is involved in the protective action of dietary saturated fat against alcoholic fatty liver in mice. J Nutr, 2008. 138(3): p. 497-501.

57. You, M., et al., Involvement of mammalian sirtuin 1 in the action of ethanol in the liver. Am J Physiol Gastrointest Liver Physiol, 2008. 294(4): p. G892-8.

58. Nascimento, A.F., et al., Aggravation of nonalcoholic steatohepatitis by moderate alcohol consumption is associated with decreased SIRT1 activity in rats. Hepatobiliary Surg Nutr, 2013. 2(5): p. 252-9.

59. Ji, C., C. Chan, and N. Kaplowitz, Predominant role of sterol response element binding proteins (SREBP) lipogenic pathways in hepatic steatosis in the murine intragastric ethanol feeding model. J Hepatol, 2006. 45(5): p. 717-24.

60. Rodgers, J.T. and P. Puigserver, Fasting-dependent glucose and lipid metabolic response through hepatic sirtuin 1. Proc Natl Acad Sci U S A, 2007. 104(31): p. 12861-6.

61. Everitt, H., et al., Ethanol administration exacerbates the abnormalities in hepatic lipid oxidation in genetically obese mice. Am J Physiol Gastrointest Liver Physiol, 2013. 304(1): p. G38-47.

62. Zeng, T., et al., CMZ reversed chronic ethanol-induced disturbance of PPAR-alpha possibly by suppressing oxidative stress and PGC-1alpha acetylation, and activating the MAPK and GSK3beta pathway. PLoS One, 2014. 9(6): p. e98658.

63. Purushotham, A., et al., Hepatocyte-specific deletion of SIRT1 alters fatty acid metabolism and results in hepatic steatosis and inflammation. Cell Metab, 2009. 9(4): p. 327-38.

64. Kong, L., et al., Activation of peroxisome proliferator activated receptor alpha ameliorates ethanol induced steatohepatitis in mice. Lipids Health Dis, 2011. 10: p. 246.

65. Thurman, R.G., II. Alcoholic liver injury involves activation of Kupffer cells by endotoxin. Am J Physiol, 1998. 275(4 Pt 1): p. G605-11.

66. Nanji, A.A., et al., Enhanced cyclooxygenase-2 gene expression in alcoholic liver disease in the rat. Gastroenterology, 1997. 112(3): p. 943-51.

67. Diehl, A.M., Recent events in alcoholic liver disease V. effects of ethanol on liver regeneration. Am J Physiol Gastrointest Liver Physiol, 2005. 288(1): p. G1-6.

68. McClain, C.J. and D.A. Cohen, Increased tumor necrosis factor production by monocytes in alcoholic hepatitis. Hepatology, 1989. 9(3): p. 349-51.

69. Khoruts, A., et al., Circulating tumor necrosis factor, interleukin-1 and interleukin-6 concentrations in chronic alcoholic patients. Hepatology, 1991. 13(2): p. 267-76.

70. Hill, D.B., et al., Increased plasma interleukin-6 concentrations in alcoholic hepatitis. J Lab Clin Med, 1992. 119(5): p. 547-52.

71. Yin, M., et al., Essential role of tumor necrosis factor alpha in alcohol-induced liver injury in mice. Gastroenterology, 1999. 117(4): p. 942-52.

72. Takeda, K. and S. Akira, Toll-like receptors in innate immunity. Int Immunol, 2005. 17(1): p. 1-14.

73. Akira, S., S. Uematsu, and O. Takeuchi, Pathogen recognition and innate immunity. Cell, 2006. 124(4): p. 783-801.

74. Seki, E. and D.A. Brenner, Toll-like receptors and adaptor molecules in liver disease: update. Hepatology, 2008. 48(1): p. 322-35.

75. Fujimoto, M., et al., Plasma endotoxin and serum cytokine levels in patients with alcoholic hepatitis: relation to severity of liver disturbance. Alcohol Clin Exp Res, 2000. 24(4 Suppl): p. 48S-54S. 
76. Fukui, $\mathrm{H}$., et al., Plasma endotoxin concentrations in patients with alcoholic and nonalcoholic liver disease: reevaluation with an improved chromogenic assay. J Hepatol, 1991. 12(2): p. 162-9.

77. Hritz, I., et al., The critical role of toll-like receptor (TLR) 4 in alcoholic liver disease is independent of the common TLR adapter MyD88. Hepatology, 2008. 48(4): p. 1224-31.

78. Kono, H., et al., ICAM-1 is involved in the mechanism of alcohol-induced liver injury: studies with knockout mice. Am J Physiol Gastrointest Liver Physiol, 2001. 280(6): p. G1289-95.

79. Nanji, A.A., et al., Intercellular adhesion molecule-1 expression in experimental alcoholic liver disease: relationship to endotoxemia and TNF alpha messenger RNA. Exp Mol Pathol, 1995. 62(1): p. 42-51.

80. Afford, S.C., et al., Distinct patterns of chemokine expression are associated with leukocyte recruitment in alcoholic hepatitis and alcoholic cirrhosis. J Pathol, 1998. 186(1): p. 82-9.

81. Marra, F., Hepatic stellate cells and the regulation of liver inflammation. J Hepatol, 1999. 31(6): p. 1120-30.

82. Rollins, B.J., Chemokines. Blood, 1997. 90(3): p. 909-28.

83. Matsushima, K., et al., Purification and characterization of a novel monocyte chemotactic and activating factor produced by a human myelomonocytic cell line. J Exp Med, 1989. 169(4): p. 1485-90.

84. Dominguez, M., et al., Hepatic expression of CXC chemokines predicts portal hypertension and survival in patients with alcoholic hepatitis. Gastroenterology, 2009. 136(5): p. 1639-50.

85. Fisher, N.C., et al., Serum concentrations and peripheral secretion of the beta chemokines monocyte chemoattractant protein 1 and macrophage inflammatory protein 1alpha in alcoholic liver disease. Gut, 1999. 45(3): p. 416-20.

86. Nanji, A.A., et al., Increased severity of alcoholic liver injury in female rats: role of oxidative stress, endotoxin, and chemokines. Am J Physiol Gastrointest Liver Physiol, 2001. 281(6): p. G1348-56.

87. Bautista, A.P., Neutrophilic infiltration in alcoholic hepatitis. Alcohol, 2002. 27(1): p. 1721.

88. Colmenero, J., et al., Hepatic expression of candidate genes in patients with alcoholic hepatitis: correlation with disease severity. Gastroenterology, 2007. 132(2): p. 687-97.

89. Lu, B., et al., Abnormalities in monocyte recruitment and cytokine expression in monocyte chemoattractant protein 1-deficient mice. J Exp Med, 1998. 187(4): p. 601-8.

90. Jiang, Y., et al., Monocyte chemoattractant protein-1 regulates adhesion molecule expression and cytokine production in human monocytes. J Immunol, 1992. 148(8): p. 2423-8.

91. Clement, S., et al., Monocyte chemoattractant protein-1 secreted by adipose tissue induces direct lipid accumulation in hepatocytes. Hepatology, 2008. 48(3): p. 799-807.

92. Kanda, H., et al., MCP-1 contributes to macrophage infiltration into adipose tissue, insulin resistance, and hepatic steatosis in obesity. J Clin Invest, 2006. 116(6): p. 1494505.

93. Mandrekar, P., et al., An essential role for monocyte chemoattractant protein-1 in alcoholic liver injury: regulation of proinflammatory cytokines and hepatic steatosis in mice. Hepatology, 2011. 54(6): p. 2185-97.

94. Szabo, G. and S. Bala, Alcoholic liver disease and the gut-liver axis. World J Gastroenterol, 2010. 16(11): p. 1321-9. 
95. Schnabl, B. and D.A. Brenner, Interactions between the intestinal microbiome and liver diseases. Gastroenterology, 2014. 146(6): p. 1513-24.

96. Turner, J.R., Intestinal mucosal barrier function in health and disease. Nat Rev Immunol, 2009. 9(11): p. 799-809.

97. Suzuki, T., Regulation of intestinal epithelial permeability by tight junctions. Cell Mol Life Sci, 2013. 70(4): p. 631-59.

98. Mitic, L.L. and J.M. Anderson, Molecular architecture of tight junctions. Annu Rev Physiol, 1998. 60: p. 121-42.

99. Mitic, L.L., C.M. Van Itallie, and J.M. Anderson, Molecular physiology and pathophysiology of tight junctions I. Tight junction structure and function: lessons from mutant animals and proteins. Am J Physiol Gastrointest Liver Physiol, 2000. 279(2): p. G250-4.

100. Aijaz, S., M.S. Balda, and K. Matter, Tight junctions: molecular architecture and function. Int Rev Cytol, 2006. 248: p. 261-98.

101. Parlesak, A., et al., Increased intestinal permeability to macromolecules and endotoxemia in patients with chronic alcohol abuse in different stages of alcoholinduced liver disease. J Hepatol, 2000. 32(5): p. 742-7.

102. Mathurin, P., et al., Exacerbation of alcoholic liver injury by enteral endotoxin in rats. Hepatology, 2000. 32(5): p. 1008-17.

103. Rao, R., Endotoxemia and gut barrier dysfunction in alcoholic liver disease. Hepatology, 2009. 50(2): p. 638-44.

104. Tamai, H., et al., Long-term ethanol feeding enhances susceptibility of the liver to orally administered lipopolysaccharides in rats. Alcohol Clin Exp Res, 2002. 26(8 Suppl): p. 75S$80 \mathrm{~S}$.

105. Mutlu, E., et al., Intestinal dysbiosis: a possible mechanism of alcohol-induced endotoxemia and alcoholic steatohepatitis in rats. Alcohol Clin Exp Res, 2009. 33(10): p. 1836-46.

106. Keshavarzian, A., et al., Preventing gut leakiness by oats supplementation ameliorates alcohol-induced liver damage in rats. J Pharmacol Exp Ther, 2001. 299(2): p. 442-8.

107. Rao, R.K., A. Seth, and P. Sheth, Recent Advances in Alcoholic Liver Disease I. Role of intestinal permeability and endotoxemia in alcoholic liver disease. Am J Physiol Gastrointest Liver Physiol, 2004. 286(6): p. G881-4.

108. Yan, A.W., et al., Enteric dysbiosis associated with a mouse model of alcoholic liver disease. Hepatology, 2011. 53(1): p. 96-105.

109. Bull-Otterson, L., et al., Metagenomic analyses of alcohol induced pathogenic alterations in the intestinal microbiome and the effect of Lactobacillus rhamnosus GG treatment. PLoS One, 2013. 8(1): p. e53028.

110. Forsyth, C.B., et al., Lactobacillus GG treatment ameliorates alcohol-induced intestinal oxidative stress, gut leakiness, and liver injury in a rat model of alcoholic steatohepatitis. Alcohol, 2009. 43(2): p. 163-72.

111. Purohit, V., et al., Alcohol, intestinal bacterial growth, intestinal permeability to endotoxin, and medical consequences: summary of a symposium. Alcohol, 2008. 42(5): p. 349-61.

112. Ferrier, L., et al., Impairment of the intestinal barrier by ethanol involves enteric microflora and mast cell activation in rodents. Am J Pathol, 2006. 168(4): p. 1148-54.

113. Bode, C., V. Kugler, and J.C. Bode, Endotoxemia in patients with alcoholic and nonalcoholic cirrhosis and in subjects with no evidence of chronic liver disease following acute alcohol excess. J Hepatol, 1987. 4(1): p. 8-14. 
114. Wiest, R. and G. Garcia-Tsao, Bacterial translocation (BT) in cirrhosis. Hepatology, 2005. 41(3): p. 422-33.

115. Guarner, C., et al., Intestinal bacterial overgrowth and bacterial translocation in cirrhotic rats with ascites. J Hepatol, 1997. 26(6): p. 1372-8.

116. Garcia-Tsao, G., et al., Bacterial translocation to mesenteric lymph nodes is increased in cirrhotic rats with ascites. Gastroenterology, 1995. 108(6): p. 1835-41.

117. Adachi, Y., et al., Antibiotics prevent liver injury in rats following long-term exposure to ethanol. Gastroenterology, 1995. 108(1): p. 218-24.

118. Hansen, J., D.L. Cherwitz, and J.I. Allen, The role of tumor necrosis factor-alpha in acute endotoxin-induced hepatotoxicity in ethanol-fed rats. Hepatology, 1994. 20(2): p. 46174.

119. Mandrekar, P., et al., The opposite effects of acute and chronic alcohol on lipopolysaccharide-induced inflammation are linked to IRAK-M in human monocytes. J Immunol, 2009. 183(2): p. 1320-7.

120. Simon, G.L. and S.L. Gorbach, Intestinal flora in health and disease. Gastroenterology, 1984. 86(1): p. 174-93.

121. Bengmark, S., Ecological control of the gastrointestinal tract. The role of probiotic flora. Gut, 1998. 42(1): p. 2-7.

122. Canani, R.B., et al., Potential beneficial effects of butyrate in intestinal and extraintestinal diseases. World J Gastroenterol, 2011. 17(12): p. 1519-28.

123. Velazquez, O.C., H.M. Lederer, and J.L. Rombeau, Butyrate and the colonocyte. Implications for neoplasia. Dig Dis Sci, 1996. 41(4): p. 727-39.

124. Hamer, H.M., et al., Review article: the role of butyrate on colonic function. Aliment Pharmacol Ther, 2008. 27(2): p. 104-19.

125. Louis, P. and H.J. Flint, Diversity, metabolism and microbial ecology of butyrateproducing bacteria from the human large intestine. FEMS Microbiol Lett, 2009. 294(1): p. 1-8.

126. Guilloteau, P., et al., From the gut to the peripheral tissues: the multiple effects of butyrate. Nutr Res Rev, 2010. 23(2): p. 366-84.

127. Davie, J.R., Inhibition of histone deacetylase activity by butyrate. J Nutr, 2003. 133(7 Suppl): p. 2485S-2493S.

128. Scharlau, D., et al., Mechanisms of primary cancer prevention by butyrate and other products formed during gut flora-mediated fermentation of dietary fibre. Mutat Res, 2009. 682(1): p. 39-53.

129. Gibson, P.R., et al., Colonic epithelial cell activation and the paradoxical effects of butyrate. Carcinogenesis, 1999. 20(4): p. 539-44.

130. Kouraklis, G. and S. Theocharis, Histone deacetylase inhibitors: a novel target of anticancer therapy (review). Oncol Rep, 2006. 15(2): p. 489-94.

131. Chen, Y.X., et al., [Regulation of histone acetylation on the expression of cell cycleassociated genes in human colon cancer cell lines]. Zhonghua Yi Xue Za Zhi, 2004. 84(4): p. 312-7.

132. Inan, M.S., et al., The luminal short-chain fatty acid butyrate modulates NF-kappaB activity in a human colonic epithelial cell line. Gastroenterology, 2000. 118(4): p. 724-34.

133. Segain, J.P., et al., Butyrate inhibits inflammatory responses through NFkappaB inhibition: implications for Crohn's disease. Gut, 2000. 47(3): p. 397-403.

134. Schreiber, S., S. Nikolaus, and J. Hampe, Activation of nuclear factor kappa $B$ inflammatory bowel disease. Gut, 1998. 42(4): p. 477-84. 
135. Gao, Z., et al., Butyrate improves insulin sensitivity and increases energy expenditure in mice. Diabetes, 2009. 58(7): p. 1509-17.

136. Daniel, P., et al., Pharmacokinetic study of butyric acid administered in vivo as sodium and arginine butyrate salts. Clin Chim Acta, 1989. 181(3): p. 255-63.

137. Miller, A.A., et al., Clinical pharmacology of sodium butyrate in patients with acute leukemia. Eur J Cancer Clin Oncol, 1987. 23(9): p. 1283-7.

138. Perrine, S.P., et al., A short-term trial of butyrate to stimulate fetal-globin-gene expression in the beta-globin disorders. N Engl J Med, 1993. 328(2): p. 81-6.

139. Sher, G.D., et al., Extended therapy with intravenous arginine butyrate in patients with beta-hemoglobinopathies. N Engl J Med, 1995. 332(24): p. 1606-10.

140. Newmark, H.L., J.R. Lupton, and C.W. Young, Butyrate as a differentiating agent: pharmacokinetics, analogues and current status. Cancer Lett, 1994. 78(1-3): p. 1-5.

141. Clifford, A.J., et al., Effects of dietary triglycerides on serum and liver lipids and sterol excretion of rats. J Nutr, 1986. 116(6): p. 944-56.

142. Su, J., et al., Evaluation of tributyrin lipid emulsion with affinity to low-density lipoprotein: pharmacokinetics in adult male Wistar rats and cellular activity on Caco-2 and HepG2 cell lines. J Pharmacol Exp Ther, 2006. 316(1): p. 62-70.

143. Egorin, M.J., et al., Plasma pharmacokinetics of butyrate after intravenous administration of sodium butyrate or oral administration of tributyrin or sodium butyrate to mice and rats. Cancer Chemother Pharmacol, 1999. 43(6): p. 445-53.

144. Jeong, W.I., et al., Paracrine activation of hepatic CB1 receptors by stellate cell-derived endocannabinoids mediates alcoholic fatty liver. Cell Metab, 2008. 7(3): p. 227-35.

145. Kirpich, I., et al., Binge alcohol-induced microvesicular liver steatosis and injury are associated with down-regulation of hepatic Hdac 1, 7, 9, 10, 11 and up-regulation of Hdac 3. Alcohol Clin Exp Res, 2012. 36(9): p. 1578-86.

146. Delcuve, G.P., D.H. Khan, and J.R. Davie, Roles of histone deacetylases in epigenetic regulation: emerging paradigms from studies with inhibitors. Clin Epigenetics, 2012. 4(1): p. 5.

147. Chen, W., Q. Yang, and R.G. Roeder, Dynamic interactions and cooperative functions of $P G C-1$ alpha and MED1 in TRalpha-mediated activation of the brown-fat-specific UCP-1 gene. Mol Cell, 2009. 35(6): p. 755-68.

148. Wallberg, A.E., et al., Coordination of p300-mediated chromatin remodeling and TRAP/mediator function through coactivator PGC-1alpha. Mol Cell, 2003. 12(5): p. 113749.

149. Ghare, S.S., et al., Coordinated histone H3 methylation and acetylation regulate physiologic and pathologic fas ligand gene expression in human CD4+ T cells. J Immunol, 2014. 193(1): p. 412-21.

150. Deacon, K., et al., Elevated SP-1 transcription factor expression and activity drives basal and hypoxia-induced vascular endothelial growth factor (VEGF) expression in non-small cell lung cancer. J Biol Chem, 2012. 287(47): p. 39967-81.

151. Aldred, A. and L.E. Nagy, Ethanol dissociates hormone-stimulated cAMP production from inhibition of TNF-alpha production in rat Kupffer cells. Am J Physiol, 1999. 276(1 Pt 1): p. G98-G106.

152. Devalaraja, M.N., et al., Increased monocyte MCP-1 production in acute alcoholic hepatitis. Cytokine, 1999. 11(11): p. 875-81.

153. Xie, G., et al., Chronic ethanol consumption alters mammalian gastrointestinal content metabolites. J Proteome Res, 2013. 12(7): p. 3297-306. 
154. Ploger, S., et al., Microbial butyrate and its role for barrier function in the gastrointestinal tract. Ann N Y Acad Sci, 2012. 1258: p. 52-9.

155. Miyoshi, M., et al., Oral administration of tributyrin increases concentration of butyrate in the portal vein and prevents lipopolysaccharide-induced liver injury in rats. Clin Nutr, 2011. 30(2): p. 252-8.

156. Cresci, G.A., K. Bush, and L.E. Nagy, Tributyrin supplementation protects mice from acute ethanol-induced gut injury. Alcohol Clin Exp Res, 2014. 38(6): p. 1489-501.

157. Peng, L., et al., Butyrate enhances the intestinal barrier by facilitating tight junction assembly via activation of AMP-activated protein kinase in Caco-2 cell monolayers. J Nutr, 2009. 139(9): p. 1619-25.

158. Caldwell, S.H., et al., Mitochondrial abnormalities in non-alcoholic steatohepatitis. J Hepatol, 1999. 31(3): p. 430-4.

159. Diraison, F., et al., Increased hepatic lipogenesis but decreased expression of lipogenic gene in adipose tissue in human obesity. Am J Physiol Endocrinol Metab, 2002. 282(1): p. E46-51.

160. Zhang, Y., et al., Peroxisome proliferator-activated receptor-gamma coactivator 1alpha (PGC-1alpha) regulates triglyceride metabolism by activation of the nuclear receptor FXR. Genes Dev, 2004. 18(2): p. 157-69.

161. Crump, N.T., et al., Dynamic acetylation of all lysine-4 trimethylated histone $\mathrm{H} 3$ is evolutionarily conserved and mediated by p300/CBP. Proc Natl Acad Sci U S A, 2011. 108(19): p. 7814-9.

162. An, W., et al., Selective requirements for histone $\mathrm{H} 3$ and $\mathrm{H} 4 \mathrm{~N}$ termini in $\mathrm{p} 300$-dependent transcriptional activation from chromatin. Mol Cell, 2002. 9(4): p. 811-21.

163. Deshmane, S.L., et al., Monocyte chemoattractant protein-1 (MCP-1): an overview. J Interferon Cytokine Res, 2009. 29(6): p. 313-26.

164. Barve, S., et al., Focus on the liver: alcohol use, highly active antiretroviral therapy, and liver disease in HIV-infected patients. Alcohol Res Health, 2010. 33(3): p. 229-36.

165. Delaney, M., History of HAART - the true story of how effective multi-drug therapy was developed for treatment of HIV disease. Retrovirology, 2006.

166. d'Arminio Monforte, A., et al., Insights into the reasons for discontinuation of the first highly active antiretroviral therapy (HAART) regimen in a cohort of antiretroviral naive patients. I.CO.N.A. Study Group. Italian Cohort of Antiretroviral-Naive Patients. AIDS, 2000. 14(5): p. 499-507.

167. Fontaine, E.A., et al., A numerical taxonomic study of anaerobic gram-negative bacilli classified as Bacteroides ureolyticus isolated from patients with non-gonococcal urethritis. J Gen Microbiol, 1986. 132(11): p. 3137-46.

168. Sherman, K.E., M. Peters, and M.J. Koziel, HIV and liver disease forum: conference proceedings. Hepatology, 2007. 45(6): p. 1566-77.

169. Nunez, M. and V. Soriano, Hepatotoxicity of antiretrovirals: incidence, mechanisms and management. Drug Saf, 2005. 28(1): p. 53-66.

170. Samet, J.H., A.Y. Walley, and C. Bridden, Illicit drugs, alcohol, and addiction in human immunodeficiency virus. Panminerva Med, 2007. 49(2): p. 67-77.

171. Bica, I., et al., Increasing mortality due to end-stage liver disease in patients with human immunodeficiency virus infection. Clin Infect Dis, 2001. 32(3): p. 492-7.

172. Johnson, A.A., et al., Toxicity of antiviral nucleoside analogs and the human mitochondrial DNA polymerase. J Biol Chem, 2001. 276(44): p. 40847-57. 
173. Barve, S., Moghe, A.,Kapoor, R., Ramirez, J.A., Eaton, J.W., Gobejishvili, L., Joshi-Barve, S. and McClain, C.J, Alcohol Use, HIV Pharmacotherapy (HAART) and Liver Disease. Alcohol Research and Health, 2010. 33: p. 229-236.

174. 2013; Available from: http://aidsinfo.nih.gov/guidelines.

175. Sham, H.L., et al., ABT-378, a highly potent inhibitor of the human immunodeficiency virus protease. Antimicrob Agents Chemother, 1998. 42(12): p. 3218-24.

176. Murphy, R.L., et al., ABT-378/ritonavir plus stavudine and lamivudine for the treatment of antiretroviral-naive adults with HIV-1 infection: 48-week results. AIDS, 2001. 15(1): p. F1-9.

177. De Clercq, E., New developments in anti-HIV chemotherapy. Biochim Biophys Acta, 2002. 1587(2-3): p. 258-75.

178. Debouck, C., The HIV-1 protease as a therapeutic target for AIDS. AIDS Res Hum Retroviruses, 1992. 8(2): p. 153-64.

179. Kohl, N.E., et al., Active human immunodeficiency virus protease is required for viral infectivity. Proc Natl Acad Sci U S A, 1988. 85(13): p. 4686-90.

180. Roberts, N.A., et al., Rational design of peptide-based HIV proteinase inhibitors. Science, 1990. 248(4953): p. 358-61.

181. Overton, H.A., et al., Effect of two novel inhibitors of the human immunodeficiency virus protease on the maturation of the HIV gag and gag-pol polyproteins. Virology, 1990. 179(1): p. 508-11.

182. Novoa, A.M., et al., Increase in the non-HIV-related deaths among AIDS cases in the HAART era. Curr HIV Res, 2008. 6(1): p. 77-81.

183. Nunez, M., Hepatotoxicity of antiretrovirals: incidence, mechanisms and management. J Hepatol, 2006. 44(1 Suppl): p. S132-9.

184. Parker, R.A., et al., Endoplasmic reticulum stress links dyslipidemia to inhibition of proteasome activity and glucose transport by HIV protease inhibitors. Mol Pharmacol, 2005. 67(6): p. 1909-19.

185. Zhou, H., et al., HIV protease inhibitors activate the unfolded protein response and disrupt lipid metabolism in primary hepatocytes. Am J Physiol Gastrointest Liver Physiol, 2006. 291(6): p. G1071-80.

186. Bruning, A., Analysis of nelfinavir-induced endoplasmic reticulum stress. Methods Enzymol, 2011. 491: p. 127-42.

187. Logue, S.E., et al., New directions in ER stress-induced cell death. Apoptosis, 2013. 18(5): p. 537-46.

188. Chakrabarti, A., A.W. Chen, and J.D. Varner, A review of the mammalian unfolded protein response. Biotechnol Bioeng, 2011. 108(12): p. 2777-93.

189. Ji, C., et al., Liver-specific loss of glucose-regulated protein 78 perturbs the unfolded protein response and exacerbates a spectrum of liver diseases in mice. Hepatology, 2011. 54(1): p. 229-39.

190. Galle, P.R., et al., Involvement of the CD95 (APO-1/Fas) receptor and ligand in liver damage. J Exp Med, 1995. 182(5): p. 1223-30.

191. Strand, S., et al., Hepatic failure and liver cell damage in acute Wilson's disease involve CD95 (APO-1/Fas) mediated apoptosis. Nat Med, 1998. 4(5): p. 588-93.

192. Taieb, J., et al., Raised plasma soluble Fas and Fas-ligand in alcoholic liver disease. Lancet, 1998. 351(9120): p. 1930-1.

193. Alkhouri, N., C. Carter-Kent, and A.E. Feldstein, Apoptosis in nonalcoholic fatty liver disease: diagnostic and therapeutic implications. Expert Rev Gastroenterol Hepatol, 2011. 5(2): p. 201-12. 
194. Peter, M.E. and P.H. Krammer, The CD95(APO-1/Fas) DISC and beyond. Cell Death Differ, 2003. 10(1): p. 26-35.

195. Purevjav, E., et al., Myocardial Fas ligand expression increases susceptibility to AZTinduced cardiomyopathy. Cardiovasc Toxicol, 2007. 7(4): p. 255-63.

196. Conti, M. and J. Beavo, Biochemistry and physiology of cyclic nucleotide phosphodiesterases: essential components in cyclic nucleotide signaling. Annu Rev Biochem, 2007. 76: p. 481-511.

197. Houslay, M.D., Underpinning compartmentalised cAMP signalling through targeted cAMP breakdown. Trends Biochem Sci, 2010. 35(2): p. 91-100.

198. Spina, D., PDE4 inhibitors: current status. Br J Pharmacol, 2008. 155(3): p. 308-15.

199. Conti, M., et al., Cyclic AMP-specific PDE4 phosphodiesterases as critical components of cyclic AMP signaling. J Biol Chem, 2003. 278(8): p. 5493-6.

200. Gobejishvili, L., et al., Chronic ethanol-mediated decrease in CAMP primes macrophages to enhanced LPS-inducible NF-kappaB activity and TNF expression: relevance to alcoholic liver disease. Am J Physiol Gastrointest Liver Physiol, 2006. 291(4): p. G681-8.

201. Jin, S.L. and M. Conti, Induction of the cyclic nucleotide phosphodiesterase PDE4B is essential for LPS-activated TNF-alpha responses. Proc Natl Acad Sci U S A, 2002. 99(11): p. 7628-33.

202. Lehnart, S.E., et al., Phosphodiesterase $4 D$ deficiency in the ryanodine-receptor complex promotes heart failure and arrhythmias. Cell, 2005. 123(1): p. 25-35.

203. Gobejishvili, L., et al., S-adenosylmethionine decreases lipopolysaccharide-induced phosphodiesterase 4B2 and attenuates tumor necrosis factor expression via CAMP/protein kinase A pathway. J Pharmacol Exp Ther, 2011. 337(2): p. 433-43.

204. Fladmark, K.E., et al., Fas/APO-1(CD95)-induced apoptosis of primary hepatocytes is inhibited by CAMP. Biochem Biophys Res Commun, 1997. 232(1): p. 20-5.

205. Li, J., S. Yang, and T.R. Billiar, Cyclic nucleotides suppress tumor necrosis factor alphamediated apoptosis by inhibiting caspase activation and cytochrome c release in primary hepatocytes via a mechanism independent of Akt activation. J Biol Chem, 2000. 275(17): p. 13026-34.

206. Webster, C.R., P. Usechak, and M.S. Anwer, cAMP inhibits bile acid-induced apoptosis by blocking caspase activation and cytochrome c release. Am J Physiol Gastrointest Liver Physiol, 2002. 283(3): p. G727-38.

207. Cullen, K.A., et al., Activation of CAMP-guanine exchange factor confers PKAindependent protection from hepatocyte apoptosis. Am J Physiol Gastrointest Liver Physiol, 2004. 287(2): p. G334-43.

208. Reinehr, R. and D. Haussinger, Inhibition of bile salt-induced apoptosis by cyclic AMP involves serine/threonine phosphorylation of CD95. Gastroenterology, 2004. 126(1): p. 249-62.

209. Gobejishvili, L., et al., Enhanced PDE4B expression augments LPS-inducible TNF expression in ethanol-primed monocytes: relevance to alcoholic liver disease. Am J Physiol Gastrointest Liver Physiol, 2008. 295(4): p. G718-24.

210. Zhou, H., et al., HIV protease inhibitors activate the unfolded protein response in macrophages: implication for atherosclerosis and cardiovascular disease. Mol Pharmacol, 2005. 68(3): p. 690-700.

211. Wang, Y., et al., The role of CCAAT enhancer-binding protein homologous protein in human immunodeficiency virus protease-inhibitor-induced hepatic lipotoxicity in mice. Hepatology, 2013. 57(3): p. 1005-16. 
212. Millott, R., E. Dudek, and M. Michalak, The endoplasmic reticulum in cardiovascular health and disease. Can J Physiol Pharmacol, 2012. 90(9): p. 1209-17.

213. Malhi, H., M.E. Guicciardi, and G.J. Gores, Hepatocyte death: a clear and present danger. Physiol Rev, 2010. 90(3): p. 1165-94.

214. Timmins, J.M., et al., Calcium/calmodulin-dependent protein kinase II links ER stress with Fas and mitochondrial apoptosis pathways. J Clin Invest, 2009. 119(10): p. 2925-41.

215. Guidelines for the Use of Antiretroviral Agents in HIV-1-Infected Adults and Adolescents.

216. Spector, A.A., HIV protease inhibitors and hyperlipidemia: a fatty acid connection. Arterioscler Thromb Vasc Biol, 2006. 26(1): p. 7-9.

217. Chen, L., et al., HIV protease inhibitor lopinavir-induced TNF-alpha and IL-6 expression is coupled to the unfolded protein response and ERK signaling pathways in macrophages. Biochem Pharmacol, 2009. 78(1): p. 70-7.

218. Koster, J.C., et al., HIV protease inhibitors acutely impair glucose-stimulated insulin release. Diabetes, 2003. 52(7): p. 1695-700.

219. Vlahakis, S.R., et al., HIV protease inhibitors modulate apoptosis signaling in vitro and in vivo. Apoptosis, 2007. 12(5): p. 969-77.

220. Flexner, C., HIV-protease inhibitors. N Engl J Med, 1998. 338(18): p. 1281-92.

221. Danner, S.A., et al., A short-term study of the safety, pharmacokinetics, and efficacy of ritonavir, an inhibitor of HIV-1 protease. European-Australian Collaborative Ritonavir Study Group. N Engl J Med, 1995. 333(23): p. 1528-33.

222. Justesen, U.S., Protease inhibitor plasma concentrations in HIV antiretroviral therapy. Dan Med Bull, 2008. 55(4): p. 165-85.

223. Gobejishvili, L., et al., Rolipram attenuates bile duct ligation-induced liver injury in rats: a potential pathogenic role of PDE4. J Pharmacol Exp Ther, 2013. 347(1): p. 80-90.

224. Liberman, A.C., et al., Underlying mechanisms of CAMP- and glucocorticoid-mediated inhibition of FasL expression in activation-induced cell death. Mol Immunol, 2012. 50(4): p. 220-35.

225. Eymin, B., et al., Increased gadd153 messenger RNA level is associated with apoptosis in human leukemic cells treated with etoposide. Cancer Res, 1997. 57(4): p. 686-95.

226. Friedman, A.D., GADD153/CHOP, a DNA damage-inducible protein, reduced CAAT/enhancer binding protein activities and increased apoptosis in 32D c13 myeloid cells. Cancer Res, 1996. 56(14): p. 3250-6.

227. Kao, E., et al., Human immunodeficiency virus protease inhibitors modulate Ca2+ homeostasis and potentiate alcoholic stress and injury in mice and primary mouse and human hepatocytes. Hepatology, 2012. 56(2): p. 594-604.

228. Hug, $\mathrm{H}$., et al., Reactive oxygen intermediates are involved in the induction of CD95 ligand mRNA expression by cytostatic drugs in hepatoma cells. J Biol Chem, 1997. 272(45): p. 28191-3.

229. Muller, M., et al., Drug-induced apoptosis in hepatoma cells is mediated by the CD95 (APO-1/Fas) receptor/ligand system and involves activation of wild-type $p 53$. J Clin Invest, 1997. 99(3): p. 403-13.

230. Hsu, S.C., et al., NF-kappa B-dependent Fas ligand expression. Eur J Immunol, 1999. 29(9): p. 2948-56.

231. Meinkoth, J.L., et al., Dynamics of the distribution of cyclic AMP-dependent protein kinase in living cells. Proc Natl Acad Sci U S A, 1990. 87(24): p. 9595-9. 


\section{CURRICULUM VITAE}

\section{HRIDGANDH DONDE}

hridgandh@gmail.com

(502) $554-4103$

505 S Hancock Street, CTRB room 552, Louisville KY 40202

\section{EDUCATION:}

2005-2008: B.S in Biochemistry (University of Mumbai, India)

2008-2010: M.S in Biochemistry (University of Mumbai, India)

2010-2013: M.S in Pharmacology and Toxicology (University of Louisville, KY,USA)

2010-2015: Ph.D. in Pharmacology and Toxicology (University of Louisville, KY,USA)

\section{POSITION:}

2013- 2015: Graduate Research Assistant (Ph.D. candidate) (University of Louisville)

\section{AWARDS:}

2010-2012 : IPIBS (Integrated Programs in Biomedical sciences) fellowship at University of Louisville.

2012-2013 : Sponsored Research Tuition Award Fall 2012-summer 2013 at University of Louisville.

2013 : Invite for platform presentation and poster presentation at Research Society on Alcoholism (RSA)

2013 : Graduate Student Travel award from American Society of Pharmacology and Experimental Therapeutics (ASPET) to attend Experimental Biology (EB) conference 
2013 : Graduate Student Best Abstract award ( $2^{\text {nd }}$ Place $)$ at The American Society for Pharmacology and Experimental Therapeutics (Division of Toxicology)

2013 : Graduate student Council travel funding (University of Louisville) to attend Society of Toxicology Conference

2013 : Graduate Student travel award from The University of Louisville School of Medicine Research Committee to attend The American Association for the Study of Liver Diseases (AASLD)

2014 : Graduate Student Travel award from American Society of Pharmacology and Experimental Therapeutics (ASPET) to attend Experimental Biology (EB) conference

2014 : Graduate Student Travel support from Society of Toxicology (SOT) to attend the conference

2014-2015 : Sponsored Research Tuition award for Summer 2014 through Spring 2015 (University of Louisville)

2015 : Graduate Student Travel award from American Society of Pharmacology and Experimental Therapeutics (ASPET) to attend Experimental Biology (EB) conference

2015 : Graduate Student Best Abstract award (Finalist) at The American Society for Pharmacology and Experimental Therapeutics (Division of Toxicology)

2015-2016 : Differential Tuition Waiver award for summer 2015 through spring 2016 (University of Louisville)

\section{Short Term Training and Courses:}

Certificate course at King Edward Memorial (KEM) Hospital, Mumbai (India) for attending the Laboratory Services at Central Clinical Biochemistry Laboratories, Emergency Laboratories, Artificial Kidney Department (AKD) (May-July 2010)

\section{PROFESSIONAL MEMBERSHIPS:}

2012- Present : Society of Toxicology (SOT) 2011- Present : Ohio Valley Society of Toxicology (OVSOT) 2011- Present : American Society for Pharmacology and Experimental Therapeutics (ASPET)

\section{TEACHING:}

2012-2013 
Participated in the training and supervision of following summer medical students fellowship project: "Hepatotoxic consequences of Highly Active Anti-Retroviral Therapy (HAART)"

1) Dino Varajic

2) Paul Page

\section{POSTER PRESENTATION:}

2011

Potential Role of FasL Expression and Signaling in the Induction of Hepatotoxicity by AZT.

Hridgandh Donde, S. Ghare, W. Chen, S. Joshi-Barve, C.J. McClain and S.

Barve. Research! Louisville, University of Louisville

2012

Ethanol is a cofactor in HAART induced hepatotoxicity

Hridgandh Donde, S. Ghare, I Kirpich, L Gobejishvili, W Chen, S Joshi-Barve, C McClain and S. Barve. Research! Louisville, University of Louisville

Ethanol is a cofactor in HAART induced hepatotoxicity

Hridgandh Donde, S. Ghare, I Kirpich, L Gobejishvili, W Chen, S Joshi-Barve, C.J. McClain and S. Barve. OVSOT (Ohio Valley Society of Toxicology)

2013

Pathogenic Mechanisms in Highly Active Anti-Retroviral Therapy (HAART) Induced Hepatotoxicity: Role of Hepatocyte Phosphodiesterase(PDE4)

Expression and ER-Stress

Hridgandh Donde, P. Barve, D. Barker, S. Joshi-Barve, L. Gobejishvile, C. McClain, S Barve

OVSOT (Ohio Valley Society of Toxicology)

Pathogenic Mechanisms in Highly Active Anti-Retroviral Therapy (HAART) Induced Hepatotoxicity: Role of Hepatocyte Phosphodiesterase(PDE4)

Expression and ER-Stress

Hridgandh Donde, P. Barve, D. Barker, S. Joshi-Barve, L. Gobejishvile, C. McClain, S Barve

Research! Louisville, University of Louisville

2014 
Pathogenic Mechanisms in Highly Active Anti-retroviral Therapy (HAART) Induced Hepatotoxicity: Role of Phosphodieaterase 4 (PDE4) and ERStress Hridgandh Donde, P. Barve, D. Barker, S. Joshi-Barve, L. Gobejishvili, C.J McClain and S. Barve Adverse Outcome Pathways workshop (AOP) of National Toxicology Program

Oral administration of tributyrin attenuates Chronic Ethanol-induced Hepatic steatosis, inflammation and injury.

Hridgandh Donde, J. Zhang, S. Ghare, L.gobejishvili, S. Joshi-Barve, V.

Vatsalya, C. J McClain and S. Barve Research! Louisville

Oral administration of tributyrin attenuates Chronic Ethanol-induced Hepatic steatosis, inflammation and injury.

Hridgandh Donde, J. Zhang, S. Ghare, L.gobejishvili, S. Joshi-Barve, V. Vatsalya, C. J McClain and S. Barve Ohio Valley Society of Toxicology (OVSOT)

\section{PEER REVIEWED ABSTRACTS}

2013

Ethanol is a Significant Cofactor in HAART Induced Hepatotoxicity Hridgandh Donde, S. Ghare, J. Zhang, I. Kirpich, S. Joshi-Barve, C. J McClain and S. Barve

Society of Toxicology (SOT)

Ethanol is a Significant Cofactor in HAART Induced Hepatotoxicity Hridgandh Donde, S. Ghare, J. Zhang, I. Kirpich, S. Joshi-Barve, C. J McClain and S. Barve

Federation of American Societies for Experimental Biology (FASEB)

Highly Active Anti-Retroviral Therapy (HAART) Induced Hepatic Steatosis and Injury Is Markedly Enhanced by Ethanol

Hridgandh Donde, S. Ghare,J. Zhang, I. Kirpich, L. Gobejishvili, W. Chen, S Joshi-Barve, C McClain and S. Barve American Association of the Study of Liver Disease (AASLD)

2014

Pathogenic Mechanisms in Highly Active Anti-retroviral Therapy (HAART) Induced Hepatotoxicity: Role of Phosphodieaterase 4 (PDE4) and ERStress

Hridgandh Donde, P. Barve, D. Barker, S. Joshi-Barve, L. Gobejishvili, C. J McMclain and S. Barve Society of Toxicology (SOT) 
Pathogenic Mechanisms in Highly Active Anti-retroviral Therapy (HAART) Induced Hepatotoxicity: Role of Phosphodieaterase 4 (PDE4) and ER-

Stress

Hridgandh Donde, D. Barker, S. Joshi-Barve, L. Gobejishvili, C. J McMclain and $S$. Barve Federation of American Societies for Experimental Biology (FASEB)

Oral administration of tributyrin attenuates Chronic Ethanol-induced Hepatic steatosis, inflammation and injury.

Hridgandh Donde, J. Zhang, S. Ghare, L.gobejishvili, S. Joshi-Barve, V. Vatsalya, C. J McClain and S. Barve

American Association of the Study of Liver Disease (AASLD) (November 2014)

2015

Tributyrin - a potential dietary supplement to target alcoholic liver disease. Hridgandh Donde, , S. Ghare, J. Zhang, S. Joshi-Barve, C. J McClain and S. Barve

American Association of the Study of Liver Disease (AASLD) (November 2014)

ORAL PRESENTATION:

Role of alcohol as a cofactor in HAART-induced hepatic steatosis \& injury (OVSOT) student summer meeting (2013)

\section{PUBLICATIONS:}

Oral administration of tributyrin attenuates Chronic Ethanol-induced Hepatic steatosis, inflammation and injury.

Hridgandh Donde, S. Ghare, J. Zhang, S. Joshi-Barve, C. J McClain and S.

Barve

(Manuscript in preparation)

Mechanistic role of PDE4/cAMP in the development of HIV-PI induced FasL mediated hepatotoxicity

Hridgandh Donde, S. Ghare, D. Barker, S. Joshi-Barve, C. J McClain and S.

Barve

(Manuscript in preparation) 
Pathogenic Mechanisms in Highly Active Anti-retroviral Therapy (HAART) Induced Hepatotoxicity: Role of Phosphodieaterase 4 (PDE4) and ERStress

Hridgandh Donde, S. Ghare, D. Barker, S. Joshi-Barve, L. Gobejishvili, C.J McClain and S. Barve. (Manuscript in preparation)

Acute exposure to acrolein, a ubiquitous environmental pollutant, and antiHIV Highly Active Antiretroviral Therapy (HAART) medication leads to hepatotoxicity.

S. Ghare, Hridgandh Donde, C.J. McClain and S. Barve and S. Joshi-Barve (Ready to submit) 